\title{
Estimating Soil Parameters Important for Lifeline Siting Using System Identification Techniques
}

Steven Glaser

Building and Fire Research Laboratory

Gaithersburg, Maryland 20899

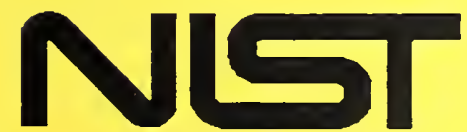

United States Department of Commerce Technology Administration

National Institute of Standards and Technology 



\section{Estimating Soil Parameters Important for Lifeline Siting Using System Identification Techniques}

Steven Glaser

March, 1993

Building and Fire Research Laboratory

National Institute of Standards and Technology

Gaithersburg, MD 20899

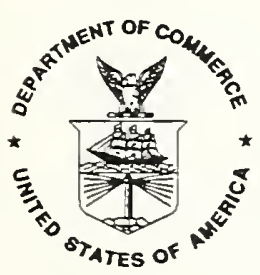

U.S. Department of Commerce

Ronald H. Brown, Secretary

National Institute of Standards and Technology

Raymond Kammer, Acting Director 



\section{ABSTRACT}

Liquefaction causes a large portion of all damage done by earthquakes. The damage is especially severe to lifeline structures such as pipelines. This report examines the state-ofthe-art of the application of System Identification (SI) methods to the liquefaction problem, with special attention to lifelines. System identification is seen as the best way to ascertain large strain soil properties in situ. A thorough introduction to SI methods and spectral analysis is given. The traditional Fourier-based methods are found to be inexact since the sample variance is equal to the sample mean if averaging techniques are not used. There is an additional problem since earthquake signals are not stationary. Autoregressive-moving average models are seen as a better analysis method, especially the newer adaptive methods that are designed for non-stationary signals. A significant bibliography is included.

KEYWORDS : ARMA modeling, building technology, earthquakes, in situ testing, lifelines, liquefaction, spectral analysis, system identification 


\section{ACKNOWLEDGEMENTS}

I would like to thank Kevin Coakley for taking the time to discuss spectral analysis and system identification with me, and for proof reading the chapter on spectral estimation. Special thanks are given to the Divisional readers Riley Chung and Tripp Shenton, and to my outside reader Prof. Will Gersch, University of Hawaii, Honolulu. I never thought I would find a volunteer to plow through this tome. Dr. Gersch has helped me learn and understand a great deal about time series analysis, and other things.

All models are incorrect, but some are more useful than others.

- G. E. P. Box 


\section{CONTENTS}

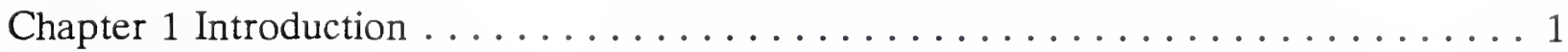

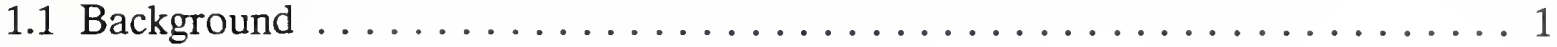

1.1.1 Earthquakes and Liquefaction . . . . . . . . . . . . . . . 1

1.1.2 Estimation Techniques and Liquefaction ................. 1

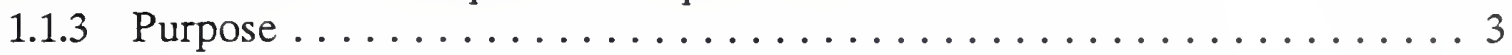

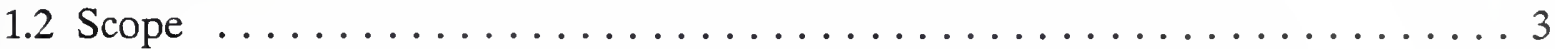

Chapter 2 Principles of Process Characterization in the Time and Frequency Domains 5

2.1 Estimates of Soil Properties from Dynamic Behavior ............ 5

2.1 .1 Introduction .......................... 5

2.1 .2 Modeling a System $\ldots \ldots \ldots \ldots \ldots \ldots \ldots \ldots \ldots \ldots$

2.2 A Traditional Approach to System Identification . . . . . . . . . . . . . 7

2.2.1 Introduction . . . . . . . . . . . . . . . . . . 7

2.2.2 Modeling Simple Mechanical Systems - Force Input and Displacement Output . ................... 8

2.2.3 Modeling Earthquake Response - Displacement Input and Displacement Output . . . . . . . . . . . . . 10

2.2.4 Evaluation of Simple System Identification Techniques . . . . . . . . . 15

2.3 Estimates of a Process in the Frequency Domain ............... 16

2.3.1 Fourier-based Methods ...................... 16

2.3.2 The DFT and Real-World Systems . . . . . . . . . . . . . . 18

2.3.3 An Improved Non-Parametric Estimator . . . . . . . . . . . . . 21

2.4 Parametric Methods of Process Estimation .................. 25

2.4 .1 Introduction . . . . . . . . . . . . . . . . . 25

2.4.2 Autoregressive (AR) Models ..................... 26

2.4 .3 Maximum Entropy Method .................... 31

2.4.4 Autoregressive-Moving Average (ARMA) Model . . . . . . . . . . . 32

2.5 Spectral Estimation . . . . . . . . . . . . . . . . . . . 35

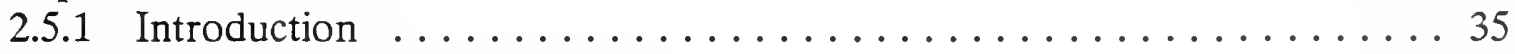

2.5.2 The Concept of a "Spectrum" . . . . . . . . . . . . . . . 37

2.5 .3 Non-stationary Signals . . . . . . . . . . . . . . . . . . 39

2.5.4 The "Instantaneous" Spectrum . . . . . . . . . . . . . . . . . . . 40

2.6 Modeling Non-stationary Processes - Adaptive Filtering . . . . . . . . . . 41

2.6 .1 Introduction . . . . . . . . . . . . . . . . . . . 41

2.6 .2 Kalman Filters . . . . . . . . . . . . . . . . . . 42 
Chapter 3 Estimation of Soil Parameters Using System Identification Techniques . . 45

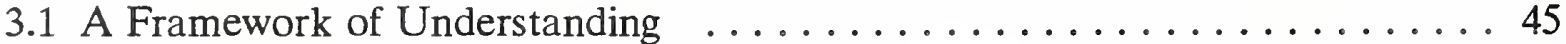

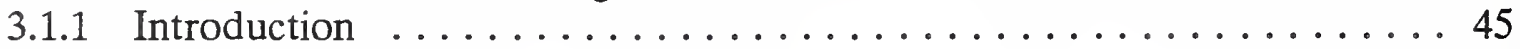

3.2 Continuous Forced Excitation of the Soil $\ldots \ldots \ldots \ldots \ldots \ldots \ldots$

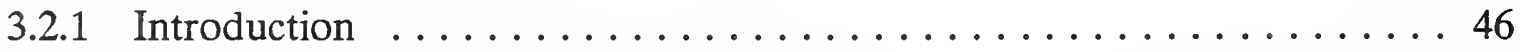

3.2.2 Applications to Multiple Layered Profiles . . . . . . . . . . . . . . . 46

3.2 .3 Modal Analysis . . . . . . . . . . . . . . . . . . . . . . . . 49

3.2.4 Estimation of Soil Properties from Impedance Functions . . . . . . . . . 49

3.3 Non-stationary Excitation of the Soil System . . . . . . . . . . . . . . 53

3.3.1 Continuous Excitation . . . . . . . . . . . . . . . 53

3.3.2 Modeling of Earthquake Strong Motion . . . . . . . . . . . . . 55

3.3.3 Transforming Non-stationary Signals into Stationary Signals . . . . . . . 59

3.3.4 Modeling of Non-stationary Processes - Time Adaptive filtering . . . . 60

3.3 .5 Summary ........................... 67

3.4 Estimation of Soil Parameters From Earthquake Strong Motion Data . . . . . 67

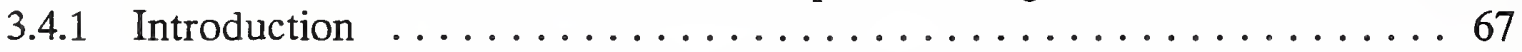

3.4 .2 Use of Shear Beam Theory .......................... 67

3.4.3 Fourier Analysis of Non-stationary Ground Motion . . . . . . . . . . . . 70

3.4.4 Parameter Estimation Using Impedance Functions . . . . . . . . . . . 70

3.4.5 Non-linear Ground Response Analyses . . . . . . . . . . . . . . 71

Chapter 4 Conclusions and Summary $\ldots \ldots \ldots \ldots \ldots \ldots \ldots \ldots \ldots \ldots \ldots$

4.1 A Framework of Knowledge ....................... 79

4.1.1 Estimation Techniques ...................... 79

4.1.2 Conclusions and Recommendations ............... 81

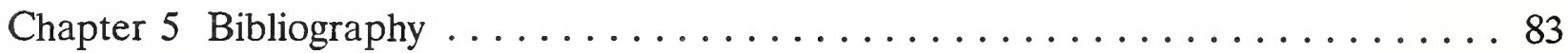




\section{CHAPTER 1 INTRODUCTION}

\subsection{Background \\ 1.1.1 Earthquakes and Liquefaction}

Over the years, some of the most spectacular, and costly damage caused by earthquakes has been due to liquefaction of sands. A functional description of liquefaction can be given quite simply. When a suitably intense earthquake shakes a loose, saturated sand, the grains tend to consolidate into a more compact packing. Since all these movements are happening very rapidly, there is no chance for the volume to reduce through pore water dissipation. The incompressible pore fluid then takes up all the applied stress, the effective stress goes to zero, and the deposit "liquefies." Since a liquid has no shear strength, disastrous consequences occur.

The disastrous consequences of liquefaction was brought to the fore in 1964 by the Niigata and Alaska earthquakes of that year. Liquefaction also triggers earth slides and large displacements of earthen dams. A large slide caused by the 1970 Peruvian earthquake killed over 18,000 people (EERI, 1986). A terrible disaster was narrowly avoided when the San Fernando dam suffered very large displacements due to the 1971 San Fernando earthquake. Similar damage has occurred over the years in locations as diverse as China, Nicaragua, Japan, Charleston, SC, San Francisco, the Imperial Valley, CA, and Idaho.

The effects of liquefaction-caused damage to lifelines are especially costly. Damage to roads, rail, telecommunications, power, and pipelines of all types is always harmful, but is especially so during time of emergency. One of the most striking examples of the effect of lifeline damage on public safety is the occurrences during the 1906 San Francisco earthquake. After that earthquake, over 490 city blocks were totally destroyed by a fire, the largest, most deadly fire in U.S. history (O'Rourke et al., 1991). Little could be done to stop the spread of the fire since the pipelines carrying water were broken due to liquefactioninduced ground displacements. It was estimated that 56 percent of the municipal water supply was completely cut off.

\subsubsection{Estimation Techniques and Liquefaction}

The reader might wonder, what does system identification and estimation of large strain soil properties have to do with liquefaction? While not immediately obvious, there are several important reasons to pay attention to these methods.

The most important piece of knowledge to be gained is that concerning the actual behavior of soils under strong motion excitation. At present, the modulus degradation and effective damping ratio curves are based only on laboratory tests. Laboratory tests will all yield at best approximate results since no one can run a laboratory test on an undisturbed 
loose sand. The preliminary reports from back calculating earthquake response imply that the laboratory degradation curve might be too high at intermediate strains. In addition, the results from two independent methods (Chang et al., 1990; Abdel-Ghaffar and Scott, 1979) show that the customary hyperbolic shape of the laboratory damping curve might be incorrect, and actually is S-shaped.

Knowledge about the interplay between pore water pressure build-up, strength of shaking, and soil nonlinearity is fundamental to rational design for earthquake loading. This interplay can only be studied by an analysis of undisturbed real-life situations using complete sets of data such as available from Lotung. It is of utmost importance for the database to be enlarged with results from other well-instrumented site. Unfortunately, there is a grave lack of such sites.

The response of soil to strong motion can be used for site characterization and microzonation (Finn, 1991). In this case, the amplification factor, or spectral ratio, is the important parameter being sought. The amplification factor is just the transfer function, which can be estimated much more accurately using the system identification methods. A simplified microzonation analysis of Charleston, South Carolina was made using SHAKE as the analysis tool (Elton and Martin, 1989), and the amplification effects of geological structures have been examined theoretically (e.g. Faccioli, 1991) and experimentally (e.g. Silva, 1989; Bard and Gabriel, 1986).

A final incentive to study in situ behavior of soils during earthquake strong motion is brought up in a late paper by Rollins and Seed (1990). This is the question of what influence structures have on their founding soils. In particular, does a structure increase or decrease the liquefaction potential, and if the soil does liquefy, will the resulting displacement be more or less than the free-field? These are important questions since the present method of analysis and design implies a free-field condition. The available data is quite scanty, based mostly on a few shaking table and centrifuge tests.

Rollins offers some conclusion as to the effects of different types of structures. These include indications that excess pore pressure ratios might be significantly lower beneath a structure, and that the soil near a foundation is more susceptible to generation of excess pore pressure than the free-field. Free field analysis appears to be too conservative for longperiod structures on medium-dense sands, and unconservative for short-period buildings on loose to medium sands.

In a wide-ranging paper, Ambraseys and Sarma (1969) give calculations showing that concentrated loads from a structure "may cause much more widespread liquefaction effects than local inhomogeneities." This is through local failure acting as seed for widespread progressive failure. On the other hand, the local increased vertical effective stress due to the structure will decrease the chance of liquefaction. Finding the balance between the two effects leads to the same questions as Rollins and Seed. 
The ideal way to find definitive conclusions to these important questions is to utilize input and output soil motions recorded in the free-field and adjacent to a structure. This data is available right now for one case - the Lotung site. A detailed analysis using state-ofthe-art system identification techniques is needed.

\subsubsection{Purpose}

This report was written to evaluate the current state-of-the-art of in situ methods of soil property measurement, which allow accurate prediction of liquefaction potential, and the possible displacements if liquefaction did occur. The report summarizes and evaluates significant technical papers on the use of system identification methods for estimating soil parameters needed to understand large strain soil behavior during earthquake loading. This topic is of direct import to the behavior of lifelines. This report makes no attempt to identify and enumerate every paper or technical publication written on these subjects. It serves, rather, as a thorough overview and evaluation of where the profession is today.

The report assumes some degree of technical sophistication by the reader, although an attempt is made to explain complicated or unfamiliar material. The liberal use of references should allow the reader to find an understandable source of explanation for most topics discussed. The report often takes a "critical" point of view when examining proposed methods. This is to examine the underlying suppositions made by a given approach, which define the validity and applicability of that method.

\subsection{Scope}

This report examines the field of system identification, and its applicability to estimating the behavior of soils undergoing strong motion. Since the field of system identification is unfamiliar to most geotechnical engineers, Chapter 2 gives an introduction to the meaning of relevant techniques. Of great importance is the thorough evaluation of the implications of spectral analysis.

Chapter 3 examines pertinent applications of system identification to liquefaction-type problems. Special attention is given to using system identification methods to estimate mechanical properties of soil undergoing intermediate-to-large strains, which can not be examined by geophysical in situ techniques.

Chapter 4 contains the important conclusions reached in this report, and briefly summarizes key concepts. A very thorough bibliography is documented in the final chapter.

Finally, it should be mentioned that the most thorough and complete work on the subject of liquefaction is the report written by the Committee on Earthquake Engineering of the National Research Council, 1985. 


\section{CHAPTER 2 PRINCIPLES OF PROCESS CHARACTERIZATION IN THE TIME AND FREQUENCY DOMAINS}

\subsection{Estimates of Soil Properties from Dynamic Behavior 2.1.1 Introduction}

A major component of the lifeline infrastructure are buried pipelines. Over the years, analytical computer programs have been developed to calculate the effects of earthquakes on these lifelines. As the programs are able to make more and more accurate predictions, the significance of the accuracy and relevancy of the input data becomes more and more important. Most of these analyses use a Winkler model, which replace the soil with springs and dashpots (Zhang et al., 1991). The relevant soil parameters become dynamic stiffness and damping. Since earthquakes excite the soil past the elastic range, estimates of the strain-dependant soil stiffness and damping are essential for further improvements in lifeline design.

Field geophysical techniques are relevant to liquefaction analysis in so far as they provide the shear-wave velocities of a site. The velocities can easily be converted to soil stiffness, or correlations with liquefaction potential derived directly for S-wave velocity. However, the shear modulus thus calculated is the small strain modulus, or $G_{\max }$, and is only valid for the elastic region of the soil. The limitation is due to the inability to reliably impart strains into the soil much greater than $1 \times 10^{-6}$. Therefore, it has been impossible to measure threshold strain, $\gamma_{T}$, and the modulus degradation curve, $G / G_{\max }$ in situ. Direct knowledge of these variables would bring the ability to make quantitative estimates of potential and possible displacements closer to reality. Another important benefit would be the possibility to measure nonlinear material damping.

Attempts to input enough energy into the ground to cause intermediate to large strains have not been very successful. The amount of energy needed would destroy a borehole, and would be destructive on the surface as well. There is also the problem of the transducers being in the near-field if they are close to a source large enough to cause large strains in an immediate area. One exception was a project undertaken for the Nuclear Regulatory Commission (Shannon-Wilson, 1976) where intermediate-to-large strains were input in a large scale field experiment.

An obvious example of large strain experiment would be the use of high explosives. While explosives have been used in the Soviet Union to estimate liquefaction parameters (Florin and Ivanov, 1961), it was done to develop a correlation with settlement, and no geophysical measurements were made. Positive results of a large in situ impulse test were reported (Shannon-Wilson, 1976). However, there has been no follow-up on this work, and other researchers have not attempted similar studies. It is not known at the present time whether that is because of lack of interest or problems with the reported method. 
The optimum situation would be the ability to make measurements during different magnitudes of earthquake excitement. In this case shear strain in the layers of interest, and stiffness (velocity), would be continually monitored. Since earthquakes can not be made-toorder, the chances of this situation happening are virtually nonexistent. The instrumentation would also be extremely difficult. However, use of inverse theory allows the soil parameters of interest to be calculated from attainable data - the ground motion records of the motion going into the layers of interest, and above the layer itself. This set of information is available for several sites (Chang et al., 1991, 1990; Holzer et al., 1989).

The modeling of a mechanical system as a transfer function calculated from known a input-output history is commonly called system identification (SI). If a suitable model is chosen to represent the system of interest, the model parameters derived will correspond to important mechanical parameters of the system, such as damping, natural frequency, and stiffness. Often times, SI is the only method available to estimate these properties, especially since the method does not actively interfere with the material properties being measured. This chapter will investigate the theory required to undertake this system identification for liquefiable soils.

\subsubsection{Modeling a System}

In the simplest case, a layer of soil can be modelled as a linear system, as diagramed in Fig. 2.1. In the time domain, the filtering process of a signal passing through this layer is represented as convolution, Eq. 2.1:

$$
y(n)=\sum_{m=0}^{n-1} x(m) \cdot h(n-m)=x * h,
$$

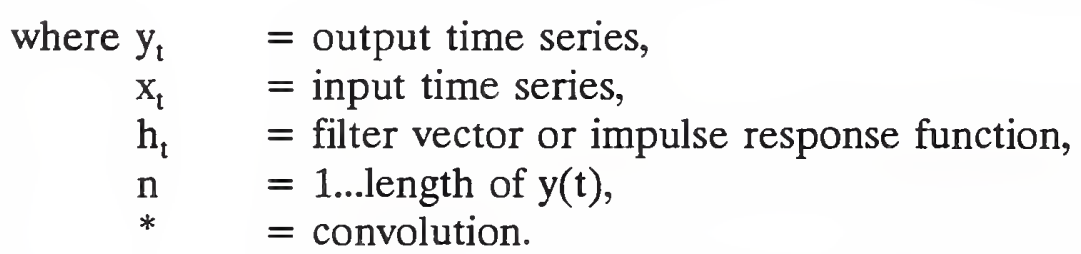

The process of inversion, or deconvolution, allows the estimation of the system response function (filter) if the input and output signals are known. Theoretically, the input and output vectors represent the coefficients of a polynomial (Z-transform) and the system response function $h_{\mathbf{t}}$ can be solved for by polynomial division (Bracewell, 1978). However, if there is any noise present, and there $A L W A Y S$ is (due to quantization error if nothing else), the quotient is irrational and frequently becomes unstable.

The usual method of time domain deconvolution is the least squares approach (Silvia and Robinson, 1979). While the deconvolution process is non-unique, the least squares method yields a system filter that is unique in a mathematical (least squared error) and 
physical sense (minimum phase). There are other, more involved, time domain solutions which are not in general use (e.g. Simmons, 1991). The time domain schemes, while possessing certain advantages, are very computation intensive. The common solution is to deconvolve in the frequency domain.

In the frequency domain, convolution becomes a simple multiplication (Bracewell, 1978), and Eq. 2.1 transforms into Eq. 2.2:

$$
Y(\omega)=X(\omega) \cdot H(\omega),
$$

where $\omega=$ circular frequency,

$\mathrm{X}_{\omega} \quad=$ frequency function of input time series,

$\mathrm{Y}_{\omega} \quad=$ frequency function of output time series,

$\mathrm{H}_{\omega} \quad=$ system frequency response function.

For this report, time domain functions will be indicated by lower case variables and frequency domain functions by upper case variables, with $\mathbf{t}$ and $\boldsymbol{\omega}$ dropped when obvious. The transformation into the frequency domain speeds computation but does not diminish the impact of noise on the calculation. If the variance of the measurement is close to the same order of magnitude as the signal energy at a given frequency, the results of the simple calculation shown in Eq. 2.2 are seriously flawed (Newland, 1984).

\subsection{A Traditional Approach to System Identification}

\subsubsection{Introduction}

As in the time domain, there is an acceptable frequency domain alternative method for computing the frequency response function using the auto and cross spectrum. Using these functions, the frequency response function is defined strictly for stationary input signals as (Bendat and Piersol, 1986):

$$
H=\frac{G_{x y}}{G_{x}}=\frac{\left(Y X^{*}\right)}{\left(X X^{*}\right)} .
$$

$$
\text { where } \begin{aligned}
G_{x} & =\text { auto-spectrum }=X X^{*}=\text { Fourier transform of } R_{x x} \\
G_{x y} & =\text { cross-spectrum }=Y X^{*}=\text { Fourier transform of } R_{x y} \\
R_{x x} & =\text { autocorrelation of } x \\
R_{x y} & =\text { cross-correlation of } x \text { with } y \\
& =\text { complex conjugate. }
\end{aligned}
$$


The use of the cross-spectrum in calculating the frequency response function is little more complicated than Eq. 2.2, but has the added benefit that the statistical variability of $\mathbf{G}_{\mathbf{x y}}$ is canceled by the variability of $G_{x}$. The real part of $H$ is called the system gain factor, while the phase information is carried by the imaginary part. The frequency response of the system is now experimentally characterized by $\mathbf{H}$, but useful mechanical parameters such as damping and stiffness are still unknown. The key is to link the frequency response function to the defining equations of the system.

\subsubsection{Modeling Simple Mechanical Systems - Force Input and Displacement Output}

The simple layered soil system in question can be characterized as a damped singledegree-of-freedom (SDOF) system. The most basic dynamic situation is with a known input force, and the resultant displacement measured, as pictured in Fig. 2.2. While this degree of knowledge is unrealistic for the earthquake system in question, it is a good model for surface excitation methods. The forces acting on the mass are given by Eq. 2.4:

$$
f(t)+f_{k}(t)+f_{c}(t)+f_{m}(t)=0
$$

and the equation of motion is

$$
m \ddot{y}+c \dot{y}+k y=f \text {. }
$$

$$
\begin{aligned}
& \text { where } f(t) \quad=\text { input force } \\
& \mathrm{f}_{\mathrm{k}}(\mathrm{t})=-\mathrm{ky} \quad=\text { spring force } \\
& \mathrm{f}_{\mathrm{c}}(\mathrm{t})=-\mathrm{cy} \quad=\text { damping force) } \\
& \mathrm{f}_{\mathrm{m}}(\mathrm{t})=-\mathrm{m} \ddot{\mathrm{y}} \quad=\text { inertial force } \\
& \mathrm{y} \quad=\text { output system displacement } \\
& \dot{\mathrm{y}}=\mathrm{dy} / \mathrm{dt} \quad=\text { velocity } \\
& \ddot{y} \quad=\mathrm{d}^{2} \mathrm{y} / \mathrm{dt}^{2} \quad=\text { acceleration. }
\end{aligned}
$$

The system response function is defined for an impulsive forcing function. The Fourier transform of the impulse response function, $\mathbf{Y}$, is the frequency response function, $\mathbf{H}$. Based on the definition of the Fourier transform and related theorems (Bracewell, 1978) the transform of Eq. 2.5, with $\mathrm{f}(\mathrm{t})=\delta_{\mathfrak{v}}$, becomes

$$
\left(-\omega^{2} m+i \omega c+k\right) Y=1
$$

where $\mathrm{i}=\sqrt{ }-1$

and the frequency response function is

Equation 2.7 is simplified by defining the damping ratio and the undamped circular natural frequency 


$$
\begin{gathered}
H=Y=\frac{1}{k-\omega^{2} m+i \omega c} . \\
\zeta=\frac{c}{2 \sqrt{k m}} \\
f_{n}=\sqrt{\frac{k}{m}}
\end{gathered}
$$

Equation 2.7 is now written as

$$
H=\frac{\frac{1}{k}}{1-\left(\frac{f}{f_{n}}\right)^{2}+i 2 \zeta\left(\frac{f}{f_{n}}\right)}
$$

For ease of analysis, the frequency response function can be broken into a system gain factor and a phase factor by writing Eq. 2.10 in polar form. The gain factor is defined as

$$
|H|=\frac{\frac{1}{k}}{\sqrt{\left[1-\left(\frac{f}{f_{n}}\right)^{2}\right]^{2}+\left[2 \zeta\left(\frac{f}{f_{n}}\right)\right]^{2}}}
$$

and the phase factor

$$
\phi=\tan ^{-1}\left[\frac{2 \zeta\left(\frac{f}{f_{n}}\right)}{1-\left(\frac{f}{f_{n}}\right)^{2}}\right] .
$$

The gain factor is shown graphically in Fig. 2.3. The gain factor for this particular forced system is called the magnification function. Note that for zero frequency the magnification 
function is the inverse of the spring constant $\mathbf{k}$. The resonant frequency $f_{r}$ can be read from the graph of the gain factor, while the fundamental frequency $\mathbf{f}_{\mathbf{n}}$ always corresponds to $a$ phase factor of $1.57 \mathbf{f}_{\mathbf{r}}$ The damping ratio can be calculated from Eq. 2.13:

$$
\zeta^{2}=\frac{1-\left(\frac{f_{r}}{f_{n}}\right)^{2}}{2}
$$

or by a simple half-power graphical technique (Richart et al., 1970). All the system parameters can be derived from the plots of the frequency response function $\mathrm{H}$ calculated by Eq. 2.3.

\subsubsection{Modeling Earthquake Response - Displacement Input and Displacement Output}

The field problem being addressed is that of a soil layer where the displacement into the layer (the bottom) and the displacement at the top of the layer are known. This system is modeled by the mechanical analog shown in Fig. 2.4. The goal is to use system parameter identification techniques to compute the soil stiffness and damping from input and output records, as in the previous example.

The equation of motion for this system is:

$$
m \ddot{y}+c \dot{y}+k y=k x+c \dot{x} .
$$

All the variables are defined as in the previous example. Applying the Fourier transform to Eq. 2.14 yields the frequency domain equation Eq. 2.15:

$$
\left(-\omega^{2} m+i \omega c+k\right) Y=(k+i \omega c)
$$

and the frequency response function $\mathbf{H}$ is defined as

$$
H \equiv Y=\frac{k+i \omega c}{k-\omega^{2} m+i \omega c} .
$$




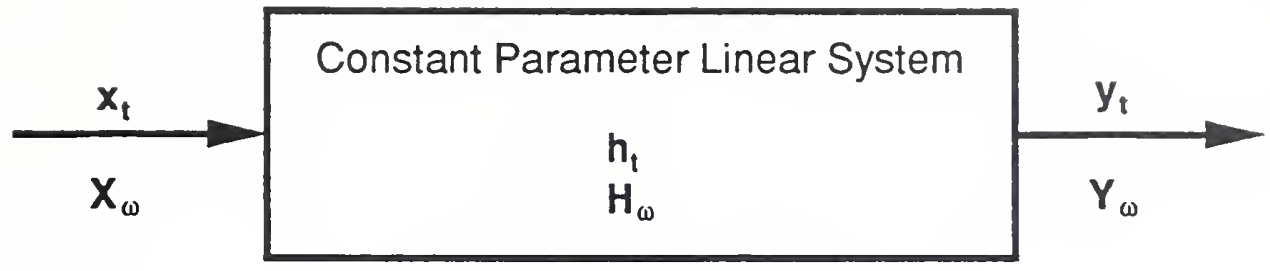

Fig. 2.1 Diagram of a linear system (filter).

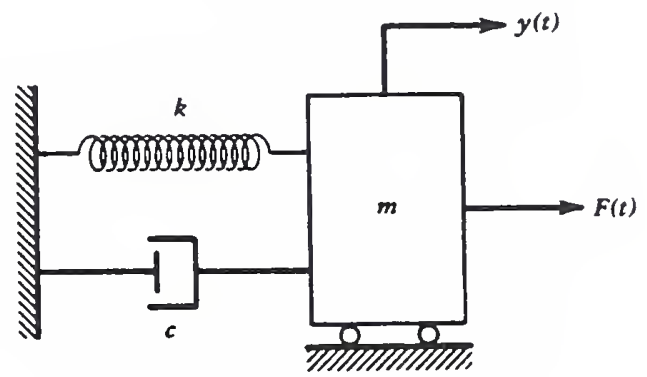

Fig. 2.2 Single-Degree-of-Freedom system, force input-displacement output (Bendat and Piersol, 1986).

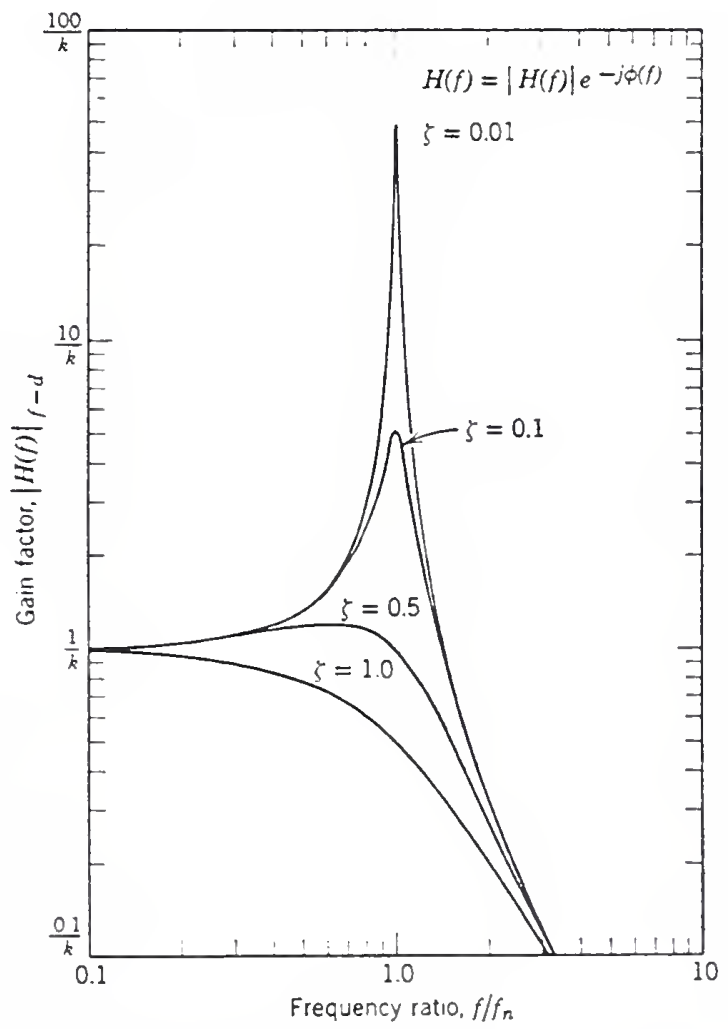

Fig. 2.3 Frequency response function (Gain Factor) of SDOF system with force input (Bendat and Piersol, 1986). 
Substituting damping ratio (Eq. 2.8) and fundamental frequency (Eq. 2.9) into Eq. 2.16 yields

$$
H=\frac{1+i 2 \zeta\left(\frac{f}{f_{n}}\right)}{1-\left(\frac{f}{f_{n}}\right)^{2}+i 2 \zeta\left(\frac{f}{f_{n}}\right)}
$$

The gain factor of $\mathrm{H}$, defined in Eq. 2.18, is called the transmissibility function since it defines how much of the forcing displacement is transmitted through the system.

$$
|H|=\sqrt{\frac{1+\left[2 \zeta\left(\frac{f}{f_{n}}\right)\right]^{2}}{\left[1-\left(\frac{f}{f_{n}}\right)^{2}\right]^{2}+\left[2 \zeta\left(\frac{f}{f_{n}}\right)\right]^{2}}}
$$

The phase factor is given by:

$$
\phi=\tan ^{-1}\left[\frac{2 \zeta\left(\frac{f}{f_{n}}\right)^{3}}{1-\left(\frac{f}{f_{n}}\right)^{2}+4 \zeta^{2}\left(\frac{f}{f_{n}}\right)^{2}}\right] .
$$

The transmissibility function is shown in Fig. 2.5.

While the graph of the gain factor for the displacement-displacement system look similar to that of the force-displacement system (Fig. 2.3), the equations are very different. From Fig. 2.5 it is seen that for any system parameters, the gain factor equals one at a frequency

$$
\omega=\sqrt{2} \cdot \omega_{n}
$$

The maximum value of the gain factor is related to the damping ratio by

$$
|H|=\frac{4 \zeta^{2}}{\sqrt{16 \zeta^{4}-8 \zeta^{2}-2+2 \sqrt{1+8 \zeta^{2}}}}
$$

which is shown in graphical format in Fig. 2.6 (Crede, 1957). For this system, values can 


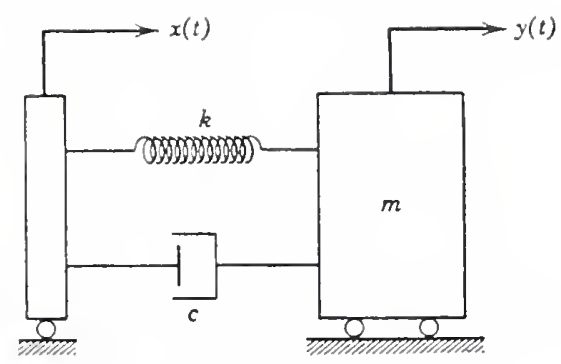

Fig. 2.4 Single-Degree-of-Freedom system, displacement input-displacement output (Bendat and Piersol, 1986).

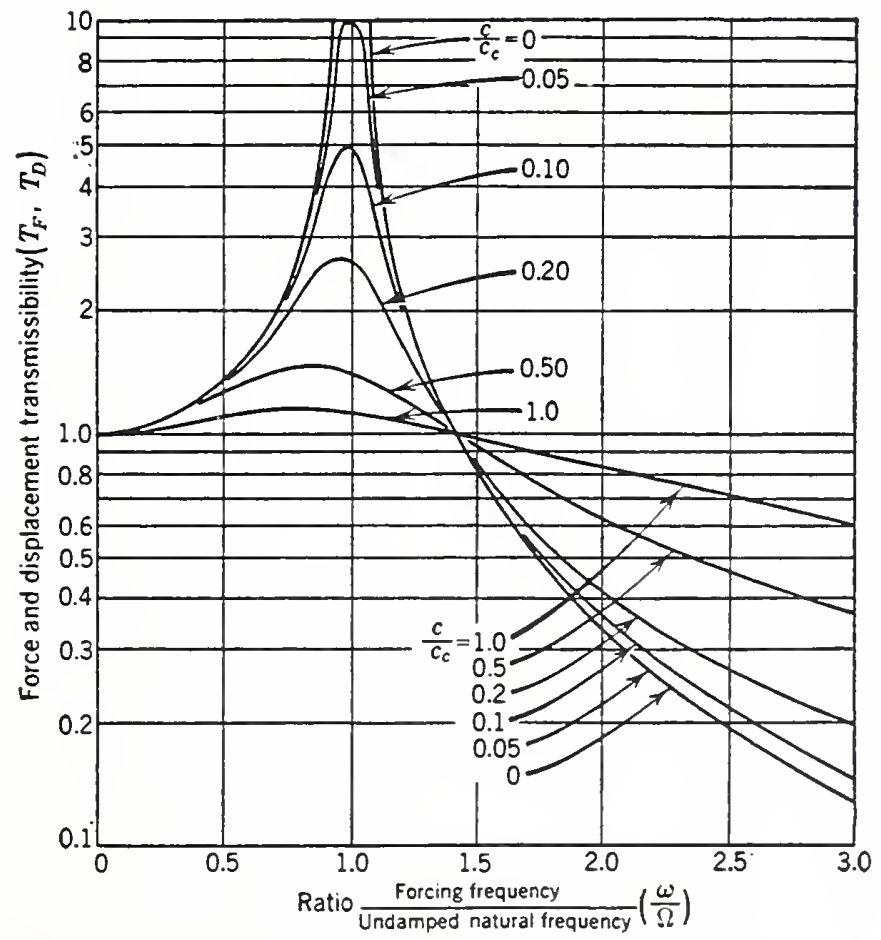

Fig. 2.5 Frequency response function of SDOF system with foundation motion input (Crede, 1951). 


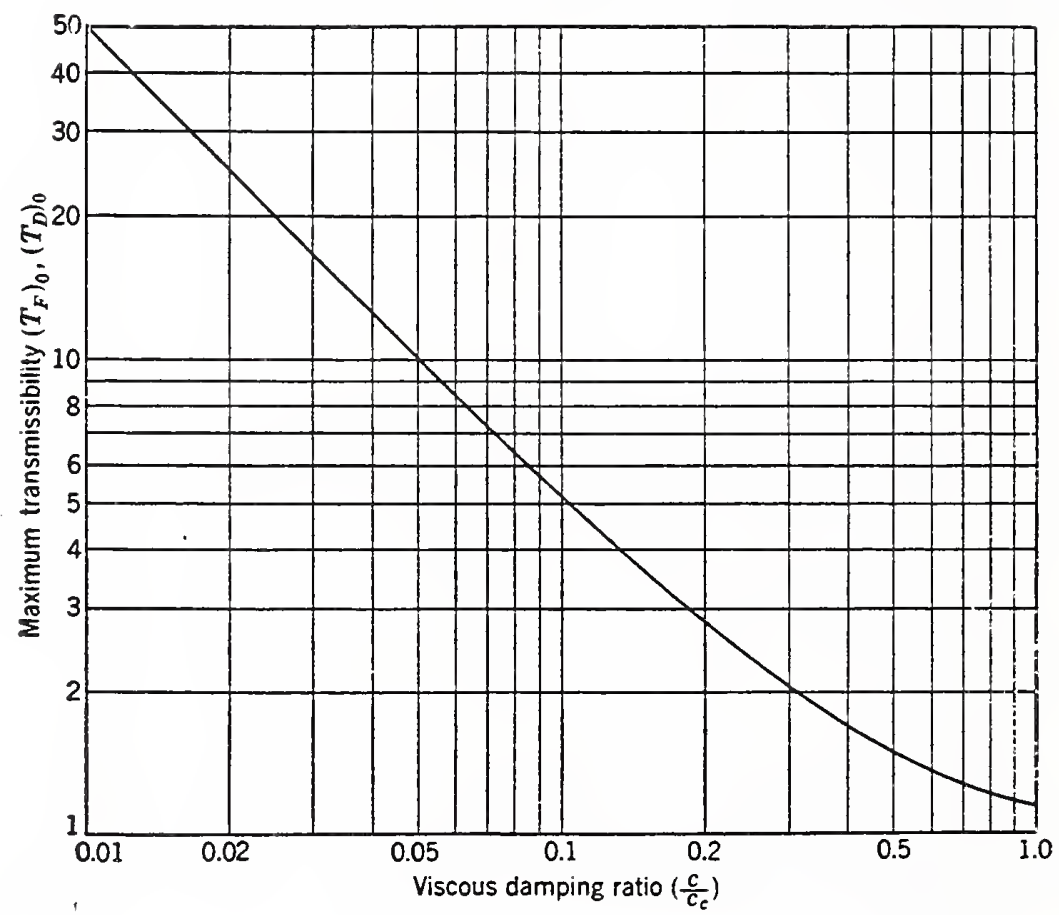

Fig. 2.6 Graphical relationship between Transmissibility and damping ratio for SDOF system with foundation motion input (Crede, 1951). 
only be derived for the damping ratio and resonant frequency. There is not enough information to directly identify the actual system stiffness and damping. In this case, mass is an unknown that prevents identification of these parameters. The concept of parameter mass is not straight-forward for a layer of soil. However, it is quite possible that the use of the mass of a unit area column of soil will provide a correct solution.

\subsubsection{Evaluation of Simple System Identification Techniques}

The inability to define all the system parameters highlights an important difficulty of the system identification method. Even for a simple SDOF system with realistic boundary conditions there is not a closed-form solution for all the basic parameters. For more complicated systems the equations get even more intractable and arcane. Often times it is not possible to find a suitable equation to define the real-world system of interest.

The solution given above is not even valid for the real-life situation of earthquake loading of a soil profile. The derivation is strictly valid for a time invariant system, which does not accurately describe a soil strained past threshold strain. The stiffness and damping of the soil are not linear, and measuring this non-linearity is one goal of utilizing system identification. In addition, the earthquake forcing function is not time-stationary, so common spectral estimation methods cannot be used to reduce effects of noise without extensive increase in the difficulty of the computation. The non-stationary methods solve for the frequency response function for input excitations that vary through time (Bendat and Piersol, 1986; Newland, 1984). For the traditional "Bendat and Piersol" approach, this involves using energy spectral density estimates rather than power spectral density estimates since the period $\mathrm{T}$ is finite rather than infinite time for which the power spectrum is defined. The single summations of Eqns. 2.3 become double summations, since the effect of change through time must be actively accounted for. The double sum estimates are not as robust as the ones for time invariant systems since the estimation are made for two stochastic variables with cumulative variances.

Assuming for the moment a time invariant system, the question is whether the excitation is stationary or not. If the input is stationary, then the cross-spectral technique can be used to define the frequency response function, minimizing the effects of noise. Stationary excitation is only to be expected for some ambient vibration tests or from controlled oscillatory sources. For these two cases the strain levels are small and the soil will behave linearly. The forcing function of the oscillatory source is known so that all system parameters can be estimated using the force displacement model. If the masses of the system are known, the problem can be solved uniquely for a multiple layered soil profile (Udwadia, 1985).

Earthquake excitation is obviously non-stationary. It is an unique event changing through time that can not ever be repeated so the effects of noise can not be eliminated through direct averaging. Because the input is non-stationary the power spectrum methods 
are strictly invalid since they depend on a statistically representative sampling of an infinite length signal. However, claim has been made that the spectral methods are equally valid for non-stationary transient data if there are a sufficient number of trials so that a valid expected value of the auto- and cross-correlations can be calculated (Bendat, 1990; Bendat and Piersol, 1986). This ensemble averaging serves to improve the statistical certainty of the estimates.

\subsection{Estimates of a Process in the Frequency Domain \\ 2.3.1 Fourier-based Methods}

At this point it is important to examine what exactly is meant by the spectral representation of a time series, and the results of the various methods of calculating the "spectrum." The most familiar form of spectral estimation is based on the Fourier transform of a continuous signal,

$$
F(\omega)=\int_{-\infty}^{\infty} f(t) e^{-i \omega t} d t
$$

This estimate is non-parametric since no particular model of process is assumed in the formulation of the estimate. However, the process is arbitrarily assumed to be representable by an orthogonal basis function of harmonic sinusoids. The basis function could just as easily be assumed to be a linear combination of damped exponentials (Prony's method), a finite number of arbitrary complex sinusoid in white noise (Pisarenko harmonic decomposition), or the output of a sharp bandpass filter centered at each frequency of interest (Capon's Maximum Likelihood Method) (see Kay and Marple, 1981).

Since $F(\boldsymbol{\omega})$ is a complex value, the following relation exists:

$$
F(\omega)=|F(\omega)| e^{i \phi(\omega)}
$$

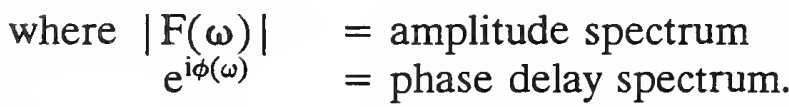

The conservation of energy between the time and Fourier domain is given by Parseval's energy theorem in terms of the square of the amplitude spectrum (Bracewell, 1978). The energy spectral density is defined as the square of the amplitude spectrum, $|F(\boldsymbol{\omega})|^{2}$, and gives the distribution of energy as a function of a set of harmonic sinusoid. The square of the amplitude spectrum is commonly called the periodogram or power spectrum (Robinson, 1982). It is related to the autocorrelation of the same time function by the WienerKhintchine theorem which states that the power spectrum is the Fourier spectrum of the autocorrelation. 
In practice, the data is available for a finite time period, and the data is in discrete form for manipulation by a digital computer. This necessitates the development of the discrete Fourier transform (DFT) (Brigham, 1974):

$$
F\left(\frac{n}{N \Delta t}\right)=\sum_{k=0}^{N-1} x(k \Delta t) e^{-i 2 \pi n \frac{k}{N}}
$$

Where

$$
\begin{array}{ll}
\mathrm{n} & =0,1,2, \ldots, \mathrm{N}-1 \quad=\text { discrete frequency counter } \\
\mathrm{N} & =\text { number of samples } \quad=\mathrm{N} \Delta \mathrm{t} \\
\mathrm{T} & =\text { digitized period } \\
\Delta \mathrm{f} & =\text { frequency resolution } 1 / \mathrm{N} \Delta \mathrm{t} \\
\Delta \mathrm{t} & =\text { time between samples. }
\end{array}
$$

The amplitude spectrum and the energy spectral density are defined as for the continuous case. However, the values for the discrete case, taken at the preassigned regular Fourier frequencies, do not match those for the analog function. This is because the sampled spectrum is actually the product of $F(\boldsymbol{\omega})$ with the sinc function (transform of the time domain boxcar windowing function which is convolved with the signal during sampling). "Thus the discrete spectrum based on a finite data set is a distorted version of the continuous spectrum based on an infinite data set." (Kay and Marple, 1981).

The unavoidable windowing of the data in the time domain, and multiplication of the spectrum with the transform of the window, leads to a smearing of the data referred to as leakage. For the simplest example of the boxcar window, the DFT representation of a simple sinusoid will be spread out over a main lobe width proportional to $1 / \mathrm{N} \Delta \mathrm{t}$ with ripples occurring at discrete intervals. These ripples are a function of the mathematics alone, and are not present in the actual infinite length analog signal. Use of other window shapes can decrease the amount of ripple, but at the expense of widening the main-lobe, thus decreasing the frequency resolution of the transform. The various windows used also have the unwanted effect of biasing the data towards the time associated with the peak of the window (Geckinli and Yavuz, 1978).

The windowing of data is based on the assumption that the portions of the times series outside the window are zero. For the case of a transient such as an earthquake seismogram, this assumption is realistic and bias is held to a minimum. For an ongoing process such as due to a mechanical oscillator, the assumption is unrealistic and the resulting spectrum will be smeared and biased. For narrow-band signals, the overlapping of adjacent side-lobes can hide the existence of close-by lower-energy components. However, for wideband processes leakage is not such an important problem. For a soil-structure interaction problem there probably would not be much difficulty identifying the first-mode resonant frequency, but other modes might be hidden. 
Reference to Shannon's sampling theorem shows that the DFT estimate is good over the frequency band of 0 to $1 /(2 \Delta t) \mathrm{Hz}$ (Brigham, 1974). It is important to note that the derivation of the DFT forces both the time and frequency domain data series to become periodic with a period $\mathrm{T}$, even if this is not the case in reality. In order to approximate the infinite length of signal utilized by the integral transform, the discrete transform implicitly assumes that the input data series is infinitely repeated, beginning to end. If the beginning and end of the data do not match and have zero slope, an infinitely quick jump is added to the data series, with attendant illegitimate high frequency energy.

\subsubsection{The DFT and Real-World Systems}

As presented by Aki and Richards (1980), a serious problem exists when estimating the amplitude spectrum when the data is "contaminated" by noise. The DFT model of the spectrum does not expressly take the presence of noise into account, and the noise must be modeled by the same harmonic sinusoids as the signal of interest. Since real field data is to be used, noise will always be present from the environment. In addition, digitized data will always have "noise" due to quantization error. The Fourier transform of the noisy data $\mathrm{N}$ is

$$
N=F X
$$

where $\mathrm{F} \quad=$ discrete transform of the signal

$\mathrm{X}=$ discrete transform of white noise.

Since the noise is a statistical variable, the linear combination $\mathbf{N}$ is also a statistical variable. Given reasonable approximations (Bloomfield, 1976), the energy spectral density $|N|^{2}$ is the sum of two squared Gaussian variables (real and imaginary parts of $N$ ) and follows the chi-squared distribution with two degrees of freedom. In this case there is little confidence that the sample Fourier spectrum is close to the "true" spectrum, since the standard deviation is now equal to the mean. There is little certainty whether an outlier is a peak value of site amplification, or acceleration, etc., or a random error.

The most common method of calculating the frequency response function was shown to be the spectral ratio, Eq. 2.2. A spectral ratio is also used to define site specific "amplification" of ground motion (Murphy et al., 1971). The spectral ratio is now seen to be a ratio of two chi-squared variables with a common mean (from a common source) and described by the Fisher F distribution with $2 \times 2$ degrees of freedom (Aki and Richards, 1980). There is very little statistical certainty with so few degrees of freedom. For this estimate there is a ninety percent probability that the spectral ratio will lie between 0.053 and 19 ! 
Simply taking more data is of no help in reducing uncertainty since the only effect is to increase the frequency resolution $\Delta \mathrm{F}$

$$
\Delta F=\frac{1}{N \Delta t} .
$$

where $\mathrm{N} \quad=$ number of digitized points

$\Delta \mathrm{t} \quad=$ inverse of digitization rate.

Digitizing at a faster rate will have the same effect. The only way to improve the certainty of the estimate of the spectrum is to average adjacent values, so that the variable now has four degrees of freedom. If $\Delta \mathrm{F}$ is very fine, many values can be averaged, giving a robust estimate of the spectrum. In order to avoid time-domain aliasing, the number of frequency values must be reduced each time adjacent frequency bins are averaged. The trade-off is confidence for frequency resolution.

When an average of adjacent values is taken, an assumption is made that the signal frequency has not changed between the two bins. Therefore, if the signal is rapidly changing, with the rate of change being on the order of the desired frequency resolution, frequency averaging can not be used and the Fourier spectrum gives a very poor estimate of the "actual" spectrum. Averaging also runs opposite to the need to maximize frequency resolution. This conundrum is the so-called Uncertainty Principle, where the frequency resolution is inversely proportional to the length of time signal, which in turn needs to be maximized to reduce variance (McClellan, 1982).

A very effective approach to improving the statistical reliability, or variance, was introduced by Welch and employs a form of ensemble averaging (Otnes and Enochson, 1978; Welch, 1967). In this method the signal is broken into blocks, the spectral estimate made for each block, and the resulting spectra averaged. If the blocks of data are overlapped, e.g. fifty percent when the Hamming window is used, the bias towards the central values is largely counteracted. This method can only be implemented if there is a large length of relatively stationary data available.

In order to compare and understand the results of the various estimation techniques discussed, it will be helpful to introduce an example data series and the actual, calculated spectrum of this process. The time history is shown in Fig. 2.7a, and is taken from Kay and Marple (1981). Figure 2.7b shows the true, calculated spectrum of this process. The process has both narrow- and wide-band information, with two line components very close together. While the spectrum looks simple, this combination is quite difficult to estimate.

Figure 2.8 shows a typical frequency spectrum calculated using the periodogram method, in this case the normalized square of the DFT. The oscillatory nature of the estimate is a result of the windowing process. Also, leakage causes broadening of the peaks which can merge the closely adjacent line spectra, shown in Fig. 2.7b. 


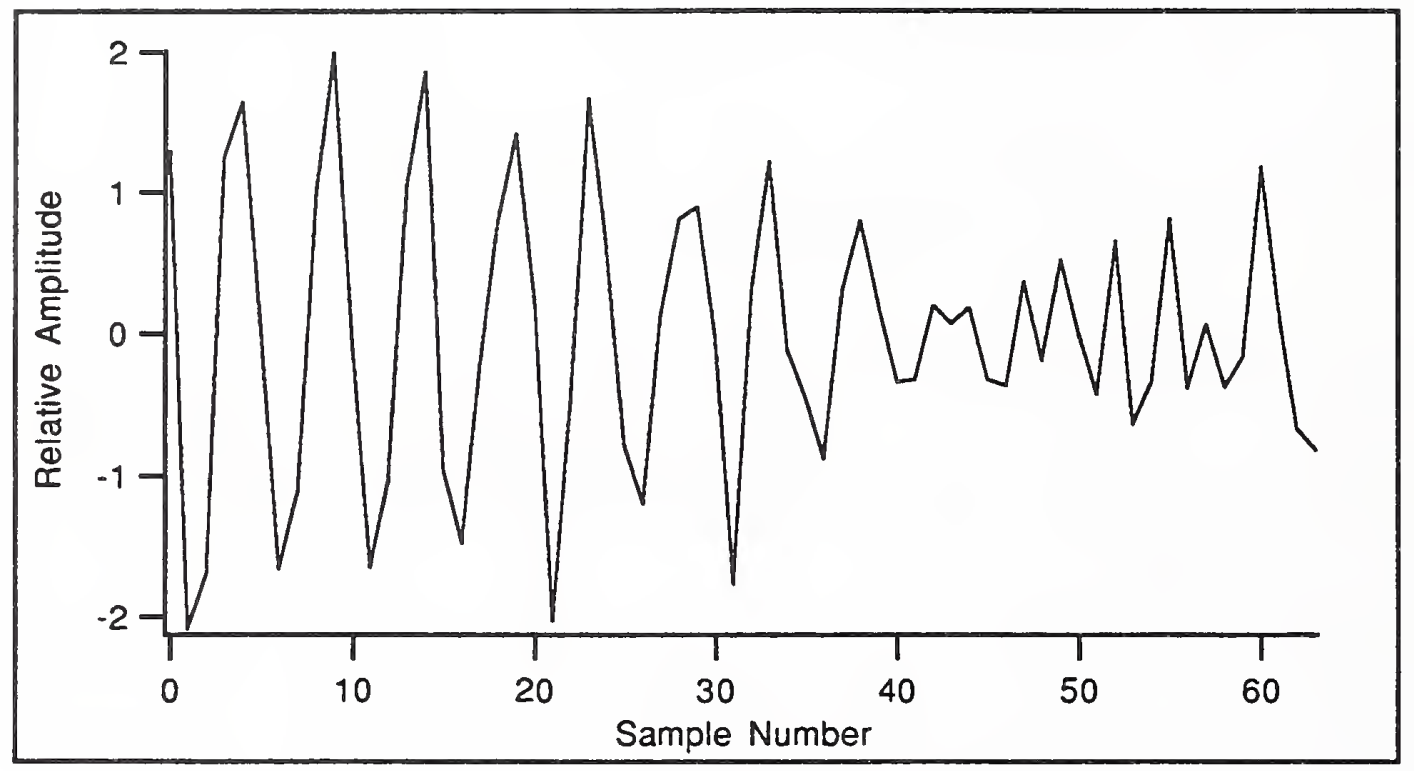

Fig. 2.7a The example data process to test spectral estimates. From Kay and Marple (1981).

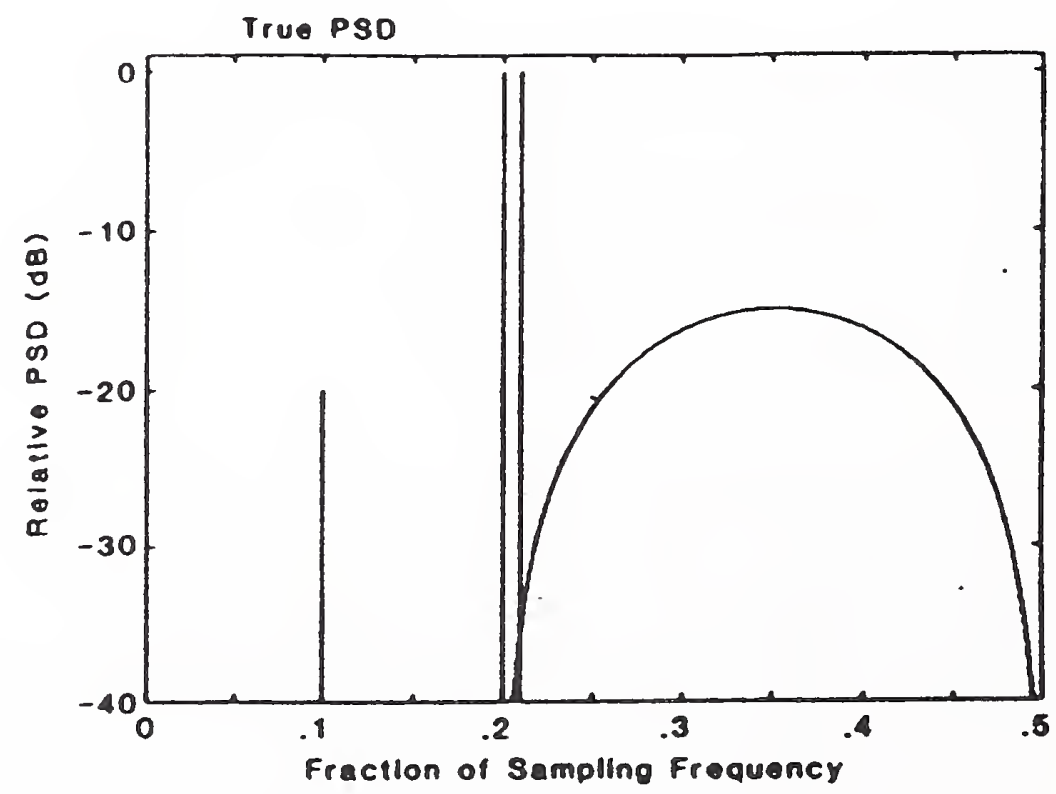

Fig. 2.7b The true, theoretical spectrim for the above test case (Kay and Marple, 1981). 
A much improved estimate is given by the Welch estimate shown in Fig. 2.9. In this case the signal has been windowed so that the large side-lobes (oscillatory shapes in Fig. 2.8) have been reduced. The variance has been greatly reduced by the averaging process. However, the two closely spaced line spectra expected around 0.2 have been merged due to the loss of resolution. In this estimate the locations of the spectral peaks are correct and the shape of the broadband portion is similar to the true spectrum.

\subsubsection{An Improved Non-Parametric Estimator}

An extremely powerful non-parametric spectral estimator for almost stationary time series has been proposed by Thomson (1982). This is the so-called multi-taper method. The method is derived for short time series which may contain line spectra as well as wide-band components. The method is rationally derived, as opposed to the ad hoc windowing and filtering of the classical approach.

In the classical method described above, the data is windowed to try to control leakage (bias), Fourier transformed, and smoothed to reduce the variance. The initial windowing increases variance and weights the data from the middle of the time series much more heavily than equally valid data from the beginning and end of the series. The smoothing (frequency averaging) is only rational if the actual spectrum is smooth. Finally, the information from the phase spectrum is discarded. The periodogram estimates are not a "sufficient statistic" of the data due to the phase information being discarded. Any finite spectral estimate is an under-determined problem (Thomson, 1982):

Since this equation \{the Fourier-based spectrum - ed \} is the frequency-domain expression of the projection from the infinite stationary sequence generated by the random orthogonal measure $\mathrm{dZ}(\mathrm{f})$ onto the finite sample, it does not have an inverse; hence it is not possible to obtain exact or unique solutions. What we desire are the statistics of those approximate solutions that are both statistically and numerically plausible.

Thomson explicitly deals with these problems by proposing a unified method which justifies the data windows used, gives consistent estimates, eliminates bias against low amplitude areas, contains separate metrics for the variance of line and broadband components, and for which the size of the time series enters into the method directly. The solution gives local independent estimates so that the spectrum at a given frequency does not depend on the spectrum at a distant frequency. Both the spectrum and the log of the spectrum are "good" estimates, while for the periodogram method the log spectrum is impossible since use of lag windows when frequency data is rapidly changing yields negative estimate values.

The multi-taper method starts with the Cramer spectral representation of a function and estimates the solution to this integral equation by a complex orthonormal eigenvector expansion. The user chooses the number of expansion terms (tapers) used: the more terms used the less biased the estimate, at the expense of frequency resolution. The class of 


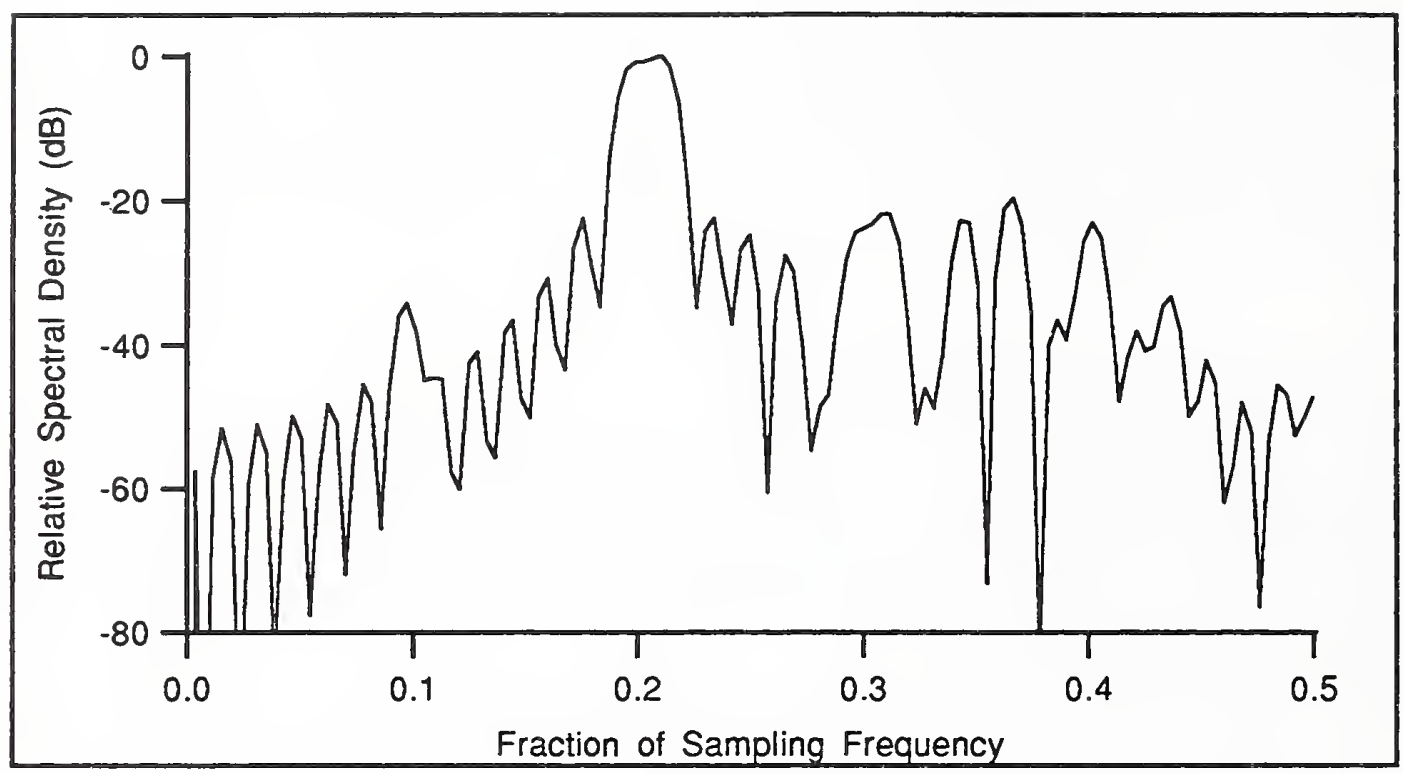

Fig. 2.8 Periodogram estimate made from the absolute square of the DFT. The typical oscillatory shape is a result of windowing.

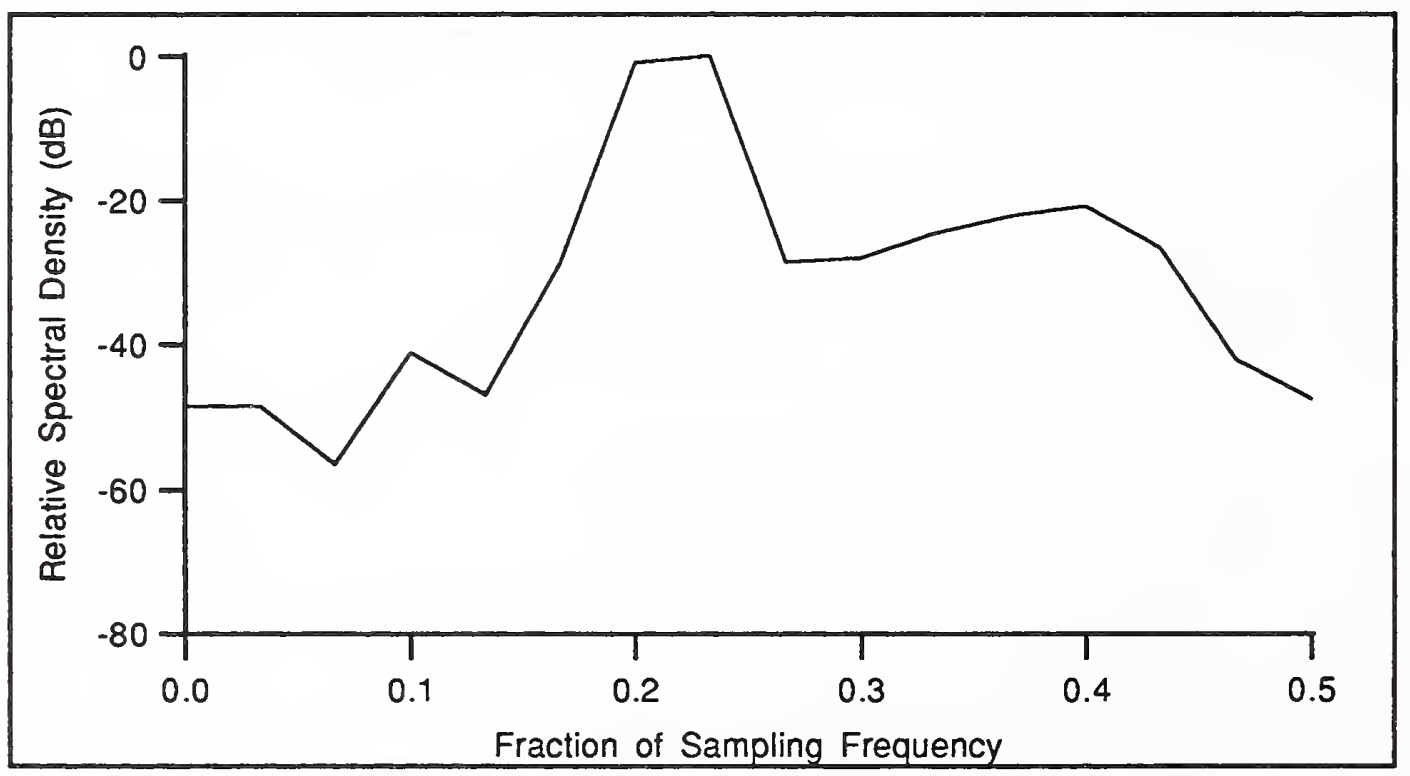

Fig. 2.9 Classical spectral estimation using the Welch method. The spectrum is to be compared to the actual spectrum shown in Fig. 2.7b. 
realizable eigenvalues is limited by weighting the expansion coefficients by prolate spheroidal wave functions, which happen to be the eigenfunctions of the Dirichlet kernel, so the method stands as a coherent whole.

The solution is reached over individual local intervals surrounding the eigenfrequencies $\mathbf{f}$, i.e. $(\mathbf{f} \pm \mathbf{w})$. The resulting estimate is chi-square distributed, but with $\left(2^{*} \#\right.$ of tapers) degrees of freedom. This is equivalent to $(4 \mathrm{Nw}) \mathrm{DOF}$, where $\mathrm{N}$ is the length of time series, and $\mathbf{w}$ is the frequency radius of interest. With the use of a reasonable number of tapers, the effective window is very rectangular over the given frequency bin, and there are very low side lobes ( $-80 \mathrm{~dB}$ for 5 tapers) effectively eliminating bias. A simple check to see if too many tapers are being used is to examine the eigenvalues in full precision; if they exceed unity, too many tapers are being used.

The method is non-parametric since the estimation is based exclusively on the time series supplied rather than on a particular model of the process producing the data. If any a priori information is known about the signal, e.g. whether the data is bandwidth-limited, or that no line spectra are present, or the exact nature of the noise, then "estimations of higher apparent resolution can be made." The power of Thomson's method is that very good estimations can be made without making imprecise a priori estimations that can skew the results towards often arbitrarily predetermined results. One of the major problems of traditional analysis is that the researcher processes and processes the data until it yields the results that were expected. However, if good prior information about the system is available, a method optimized for the particular situation should give results superior to those from a general solution.

The power of this method for a knowledgeable user is shown in Fig. 2.10, which is from Thomson's paper (1982). The estimate for the test time series is for all practical purposes the same as the true spectrum. However, Thomson did much more than just apply his algorithm, which gives the poor result shown in Fig. 2.11. Thomson utilized the following multi-step estimation procedure.

(1) Apply the multi-taper algorithm.

(2) Calculate the variance using the F-statistic and identify the frequencies of suspected line components. This step takes a fair amount of experience and knowledge. Thomson makes decisions that a user of lesser experience might not be able to make.

(3) Subtract the effects of the line components.

(4) Further pre-whiten the spectrum with an autoregressive (AR) prediction error filter. Thomson uses a fifth-order filter. Pre-whitening is a method used to remove bias from the estimate (Hardin, 1986; Newland, 1984). By removing spectral peaks, bias due to leakage is reduced since bias is proportional to the second derivative of the spectrum. In addition, since the variance of the estimate is proportional to the mean of the estimate, making the estimate smaller reduces the error. By making the spectral estimate flat - white - bias is reduced towards zero and the variance is constant for all frequencies. An initial spectral estimate is made to locate peak 


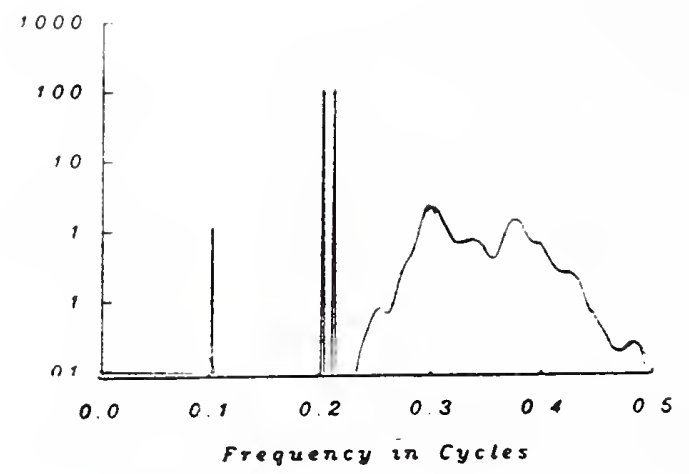

Fig. 2.10 Spectral estimation of the test data made by Thomson using his multi-taper method and preprocessing (Thomson, 1982).

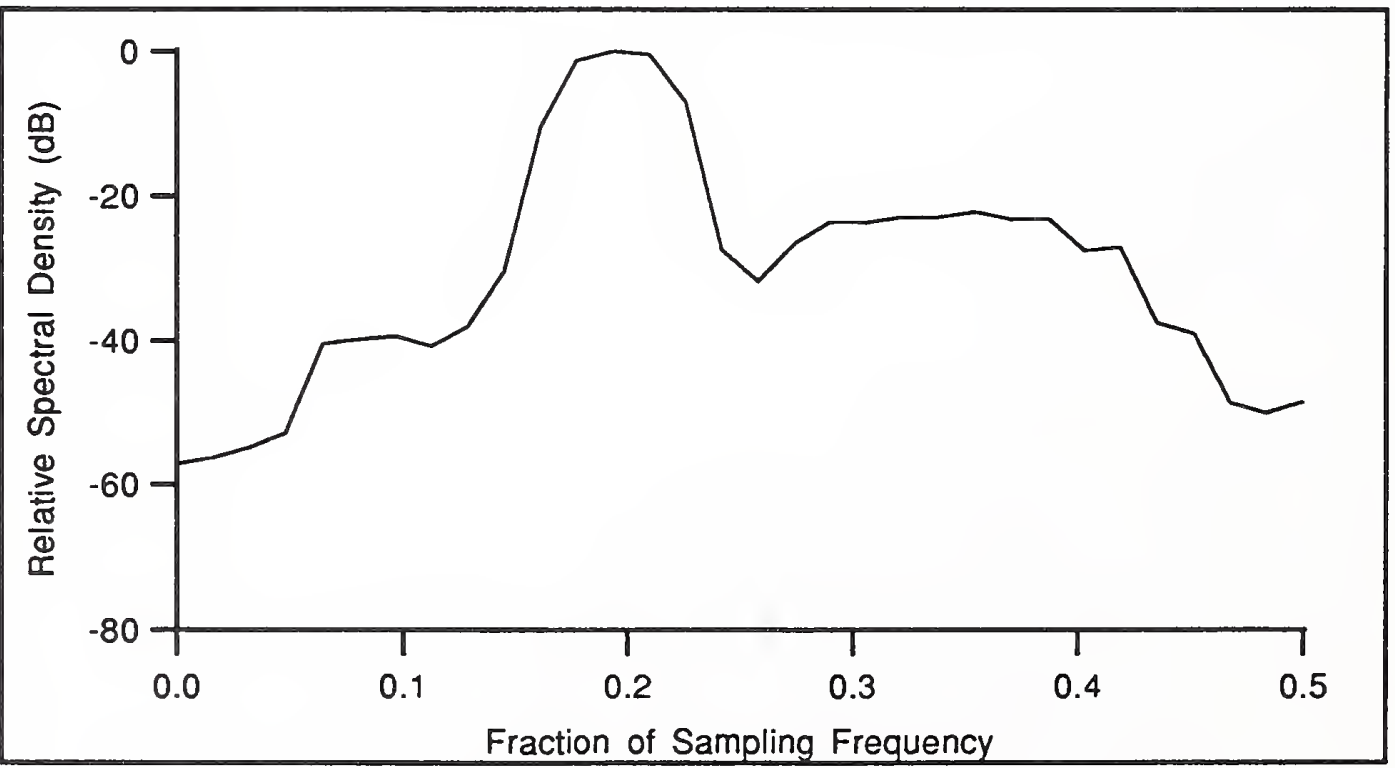

Fig. 2.11 Spectral estimate of the test data made by applying multi-taper method with no preprocessing. 
frequencies, a filter designed to remove these peaks (prediction error filter), new spectral estimate made, and the estimate postdarkened by recombination of the removed peaks.

(5) Residual spectrum postdarkened and the line components added, giving the final estimate.

\subsection{Parametric Methods of Process Estimation}

\subsubsection{Introduction}

For the non-parametric approach to spectral estimation, no assumption was made about the nature of the data except that the time series values are identically zero outside the windowed section. This condition is true for a complete transient, but obviously is not for a stationary segment of a transient. Very often some information is known about the signal or the source. It might be something as simple as the fact that the frequency content of the region of interest is band-limited. For example, the resonant frequency of a building is known to lie within a narrow frequency range. Use of a priori information can allow a very appropriate model of the process to be used to estimate a very accurate spectrum with a small amount of data.

The energy spectral density, as represented by the standard periodogram approach discussed above, can be shown to be identical to a parametric model of a least squares fit of the time series to a simple harmonic model — the DFT (Kay and Marple, 1981). The discrete Fourier frequencies are preassigned, as well as the number of frequency bins, based on the digitized period and digitization speed used to sample the data. In addition, noise is not accounted for in the model, the energy of which is included in the frequency estimates. The effects of noise must be removed through the various averaging schemes discussed.

In the above discussion of Thomson's approach, it was pointed out that an unresolvable limitation of the classical DFT approach to spectral estimation is the fact that a finite set of values (the frequency domain time series) and observations are used to represent a function in actuality continuous in both the time and frequency domains. In the parametric approach, a model with a finite number of parameters characterizes the process. The recorded data is used to estimate the parameters of the chosen model. Note that there is an implicit requirement that the model be a good representation of the actual physical process being studied. 


\subsubsection{Autoregressive (AR) Models}

2.27

The most common approach is to model the system as a linear filter, given by Eq.

$$
y_{t}=b_{0} x_{t}+b_{1} x_{t-1}+\cdots+a_{1} y_{t-1}+a_{2} y_{t-2}
$$

$$
\begin{array}{cl}
\text { where } \mathrm{y}_{\mathrm{j}} & =\text { actual data sequence (modeled as the filter output) } \\
\mathrm{x}_{\mathrm{j}} & =\text { system input sequence (assume white noise for spectral estimation) } \\
\mathrm{t} & =\text { time step counter. }
\end{array}
$$

The output is seen as a combination of the input white noise history acted upon by the "b" coefficients plus the past outputs acted upon by the "a" coefficients. The input series, involving the " $\mathrm{b}$ " coefficients, is a causal moving average (MA) process (convolutional). The series involving weighted past output values ("a" coefficients) is a noncausal autoregressive (AR) process. The lengths of the MA and AR processes must be explicitly chosen so that the model best represents the actual process (an additional piece of required information compared to the DFT).

In order to solve for the model parameters, Eq. 2.27 is rearranged to give

$$
b_{0} x_{t}+b_{1} x_{t-1}+\cdots=y_{t}+a_{1} y_{t-1}+a_{2} y_{t-2}+\cdots=\sum_{k=0}^{q} b_{k} x(t-k)=y_{t}+\sum_{k=1}^{p} a_{k} y(n-k)
$$

The Fourier transform of Eq. 2.28 is taken by applying the shifting theorem to yield

$$
Y_{\omega}\left(1+a_{1} e^{1 i \omega}+a_{2} e^{2 i \omega}+\cdots\right)=X_{\omega}\left(b_{0}+a_{1} e^{1 i \omega}+b_{2} e^{2 i \omega}+\cdots\right)
$$

Applying the Z-transform, where $z^{k}=e^{i \omega t}$, to Eq. 2.29, and rearranging, gives

$$
H_{\omega}=\frac{Y_{\omega}}{X_{\omega}}=\frac{b_{0}+b_{1} z^{1}+b_{2} z^{2}+\cdots}{1+a_{1} z^{1}+a_{2} z^{2}+\cdots}
$$

The amplitude spectrum of the actual data now becomes

$$
\left|Y_{\omega}\right|=\left|\frac{b_{0}+b_{1} z^{1}+b_{2} z^{2}+\cdots}{1+a_{1} z^{1}+a_{2} z^{2}+\cdots}\right|,
$$

since the amplitude spectrum of the white noise input $\mathbf{X}_{\omega}$ is a constant equal to one. The numerator polynomial (in $\mathrm{Z}$, order $\mathrm{q}$ ) is the MA process while the denominator polynomial contains the AR coefficients. 
If a process is thought to be a function only of the white noise input being acted upon by the filter model, the process is said to be a moving average process. The denominator of Eq. 2.31 becomes identically one, and the roots of the factored numerator (MA) polynomial are all "zeros" of the equation. If the roots are close to the unit circle, then the amplitudes of the frequencies close to the zeros will become very small. The spectrum will be able to model smooth spectra with sharp notches very well, but will poorly define sharp peaks unless a very large number of parameters are solved for. The AR model is popular since it can describe a complex process with very few parameters calculated from a small length of data, and there have been many improvements of the estimation procedure.

The standard periodogram approach to spectral estimation can be shown to be a special case of the MA process (Cadzow, 1982). This is reflected in the fact that for the time domain analog, the autocorrelation (from the Weiner-Khintchine theorem), it is only possible to calculate a finite number of time lags $(q+1)$ for finite data. The autocorrelation was assumed to be zero outside the time of interest, with attendant leakage problems. For broad-band signals, this assumption is "almost correct", and it was just shown that a MA estimation would work very well for such a process.

Since any parametric approach is useful only if it actually is a representative model of the data process, it is important to develop a feel for AR processes. It has been shown above that an AR process is a feedback process, described in the time domain as

$$
y_{t}=x_{t}-a_{1} y_{t-1}-a_{2} y_{t-2}-\cdots
$$

The current output of the model is a function of the current input (assumed to be random for spectral estimation) and a weighted sum of the past outputs. In this sense, the AR filter can be seen to model discrete integration, combining past outputs, while the MA filter models a difference (Robinson, 1982).

Robinson (1982) describes the derivation Yule used in 1927 (Yule, 1927) to formulate the AR spectrum. Yule imagined a simple, damped SDOF oscillator - in this case an airdamped pendulum. This system can be described in a discrete sense by the homogeneous difference equation

$$
c(t)+a_{1} c(t-1)+a_{2} c(t-2)=0
$$

where $\mathrm{c}(\mathrm{t}) \quad=$ displacement amplitude at time $\mathrm{t}$

$\mathrm{a}_{\mathrm{i}}=$ model parameters, $\mathrm{a}_{2}$ is the reflection coefficient. 
The solution to Eq. 2.33 can be shown to be

$$
c_{t}=e^{\lambda t} \frac{\sin (t+1) \omega_{0}}{\sin \omega_{0}}
$$

where

$$
\begin{gathered}
\lambda=\frac{1}{2} \ln \left(a_{2}\right) \\
\omega_{0}=\tan ^{-1}\left(\frac{-\sqrt{4 a_{2}-a_{1}^{2}}}{a_{1}}\right) .
\end{gathered}
$$

and $c_{t} \quad=$ impulse response function

$\omega_{0} \quad=$ fundamental frequency of the impulse response function.

This result should be compared to Eq. 2.3, where an equivalent system was modeled from mechanical principles rather than by a parametric feedback process.

The pendulum is then excited by a white noise driving function. Yule postulated several boys with pea shooters irregularly pelting the pendulum from different locations. The graph of the motion of the pendulum through time will be quite smooth, with the amplitude and phase varying continuously as given by

$$
y(n)+a_{1} y(n-1)+a_{2} y(n-2)=x(n)
$$

where $x_{t} \quad=$ white noise input excitation.

The solution for Eq. 2.37 is

$$
y(t)=\sum_{k=0}^{N} c(k) x(t-k)
$$

where $\mathrm{N} \quad=$ number of measurements of amplitude taken

$\mathrm{c}(\mathrm{t}) \quad=$ impulse response function given by Eq. 2.34.

The model uses a finite number of chosen parameters, $a_{1}$ and $a_{2}$. Using the data series $y(t)$, the coefficients are found by regressing $y_{t}$ on the past of $y_{t}-$ self-regression or autoregression. The solution utilizes the least squares normal equations filled with the empirical autocorrelation values for the data series, and are called the Yule-Walker equations. They result in a Toeplitz matrix and the equations are rapidly solved using Levinson recursion (Levinson, 1947). 
The AR coefficients can now be used to estimate both the amplitude and phase spectra of the data series. The AR coefficients model the process in the time domain - they model the time series. The frequency response of the model, i.e. filter, can be realized by transforming the model parameters. Evaluating the time domain results around the unit circle, i.e. taking the Z-transform, yields

$$
C_{\omega}=\sum_{n=0}^{N} C(n) z^{n}=\frac{1}{1+a_{1} z+a_{2} z^{2}}=\frac{1}{1+e^{i 1 \omega}+e^{i 2 \omega}} .
$$

The AR model is much different than the MA-type approach made by the periodogram, which assumed a sinusoidal process with added white noise. If the amplitude of the white noise increases, the graph will look confusing, but the periodogram will do a fairly good job (given enough observations) of picking out the sinusoid. However, the improved resolution given by the AR approach is dependant on the signal-to-noise ratio between the sinusoid and background white noise, doing a poor job in noisy environments (Marple and Lawrence, 1987)

For the AR case, the roots of the factored AR polynomial are all "poles." For frequencies adjacent to poles near the unit circle, amplitudes will be very large, reminiscent of the shape of a circus tent close to the poles. If it is expected that the spectrum is dominated by sharp spikes, then the process can be well modeled as an autoregressive process.

A serious problem can occur if the actual autocorrelation is not zero outside the limited number of lags available. This problem occurs when the process is actually made up of sinusoid and white noise. A more suitable model for this system would be one that does not window the signal, i.e. does not violently truncate the autocorrelation. The most common such model is the AR model. This model is merely Eq. 2.31 with the numerator terms are set to zero, except for the zero time lag which is unity (from autocorrelation of the input white noise series $x_{1}$ ).

Insight into the above discussion is given in Fig. 2.12 (Marple and Lawrence, 1987). Figure 2.12a shows the actual, complete autocorrelation for a single sinusoid on the left, and the true power spectral density for a single sinusoid on the right. The assumptions of the periodogram method, and the realities of data-limited discrete processing, yield the truncated, windowed, autocorrelation shown on the left of Fig. 2.12b. The matching biased, inconsistent, periodogram estimate is shown on the right. Finally, the left side of Fig. 2.12c shows the effective extrapolation to the autocorrelation made by the very simple YuleWalker solution for the AR coefficients. This extrapolation results in the sharp, unbiased estimate shown on the right side of Fig. 2.12c.

Unfortunately, there are an infinite number of valid extrapolations of the truncated autocorrelation. The Yule-Walker approach (Marple and Lawrence, 1987) to estimating the AR model parameters is based strictly on the truncated autocorrelation and does not extract the maximum information out of the available data. 


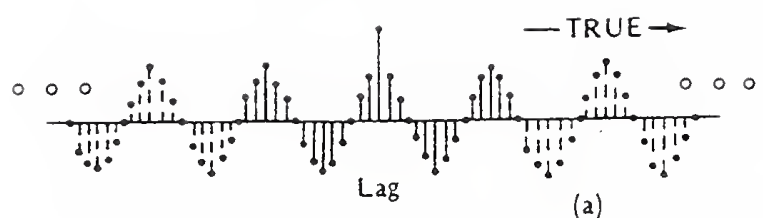

(a)
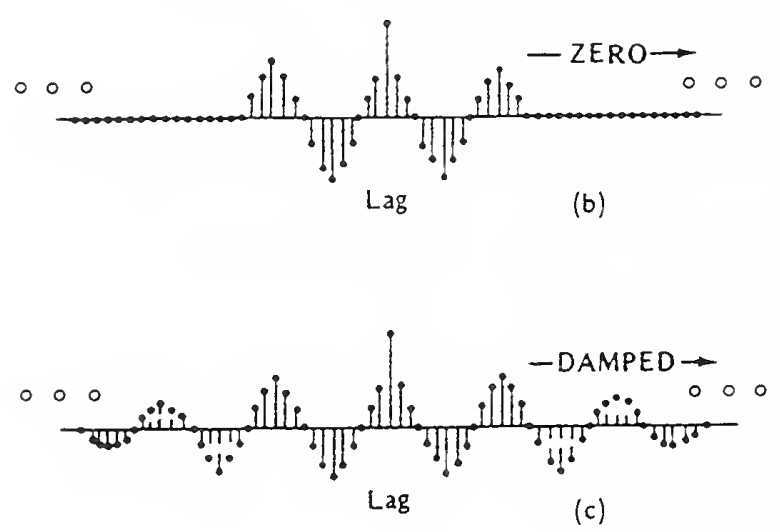
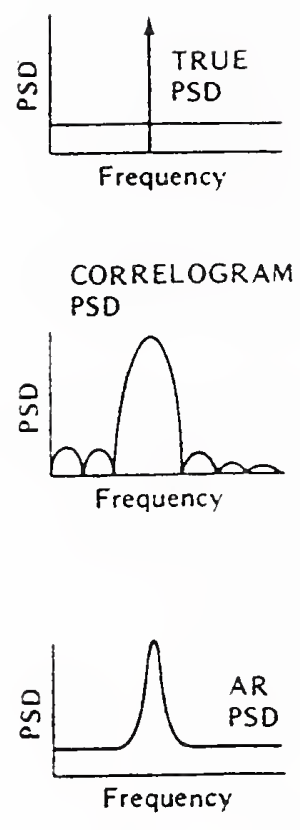

Fig. 2.12 Burg's extrapolation of the autocorrelation series (ACS): (a) actual ACS and the true spectrum (b) truncated and incomplete ACS due to limited discrete data and the classical estimation (c) Burg's ACS extrapolation and improved AR spectral estimation (Marple, 1987). 


\subsubsection{Maximum Entropy Method}

The Burg algorithm (Burg, 1975) is the most popular method for estimating an AR process. This is the so-called Maximum Entropy estimation. As for all AR methods, the spectral estimation process is two-fold. The first step is the calculation of the model parameters from the recorded data. This is done in the time domain, and is where the effective extension of the autocorrelation manifests itself. The second step is to transform the AR coefficients into the spectral estimate. However, the Burg approach estimates the $\mathrm{AR}$ parameters without making any explicit estimates of the autocorrelation.

In the time domain, there are only a limited number of lags of the autocorrelation known, and an infinite number of possible extrapolations. The problem is to choose the "best" representative of this infinite class. Burg chose the spectrum which corresponds to the most random time series whose autocorrelation matches that of the actual data series. Burg generates a new data set, based on the actual data, which is the most random possible in an entropy sense. The reasoning is that this imposes the fewest possible constraints on the solution, minimizing the bias. The extrapolation supplies additional information so that the estimate has very high resolution compared to other methods, and is optimal for short data sets (Hardin, 1986).

In this case, the concept of entropy has to do with measuring the information content of a "message", or combination of symbols (Shannon, 1948). Maximizing information is the same as maximizing choice when selecting a message; and the more freedom, the more uncertainty as to the outcome (Radoski et al. 1975). The entropy of a distribution is maximum when all probabilities are equal. Since Burg's new data set has the most randomness (maximum entropy), the associated spectral estimate has a high resolution since it uses all the possible information contained in the estimated autocorrelation, when extrapolating beyond the limited number of lags to the "true" autocorrelation.

Radoski states that the entropy function to be maximized is

$$
E=\int_{-\omega_{0}}^{+\omega_{0}} \operatorname{Ln}[S(\omega)] d \omega
$$

where $\mathrm{E} \quad=$ entropy function

$\mathrm{S}(\omega) \quad=$ spectral density

$\omega_{0} \quad=$ Nyquist frequency, $\pi / \mathrm{N} \Delta \mathrm{t}$ radians

$\Delta \mathrm{t} \quad=$ digitization interval.

The problem to be solved is to calculate a spectrum that maximizes the entropy function $\mathrm{E}$, subject to the constraints of the actual, lag-limited, autocorrelation estimate. For an infinite data series, the Burg estimation and the periodogram would be identical. Rather than use the (incomplete) autocorrelation estimates to calculate the AR parameters, i.e. the Yule- 
Walker equations, Burg uses a least squares approach to minimize the forward and backwards prediction error with respect to the last filter element $\mathrm{a}_{\mathbf{n}}$, and solving for the remainder of the parameters by Levinson recursion. A very important advantage of Burg's technique is that it is optimized for use of a very small number of data points to yield a robust system estimate.

Since the introduction of the Burg method, many other estimation schemes have been proposed to improve on limitations. Swingler (1979) points out that the bidirectionality constraint limits the application of Burg's method. Not all deterministic signals, such as exponential decays, can be successfully modeled. This failing should not be too surprising since Burg's derivation was for stationary signals. Swingler also states that the insistence on using Levinson recursion causes small frequency shifts for sinusoid under certain phase conditions. Swingler presents a non-recursive least squares estimate that is functionally identical to that proposed by Ulrych and Clayton (1976).

Spectra estimated by the AR models have a typical swept-peak shape. The peaks are located at the correct places, but their shape is not a function of the signal itself. In fact, the number of peaks calculated is a function of the AR order chosen by the user. There will be approximately one peak for every two parameters chosen, which follows from Yule's derivation. The typical AR spectrum shown in Fig. 2.13a exhibits the "peaky" shape and good estimation of sharp peaks typical of the AR estimate. In contrast, Fig. 2.13b shows the sharp drops well modeled by the MA spectral estimate. As expected, the combined AR-MA estimation is a combination of the two. Note that the shape of the peaks is a function of the estimation procedure chosen and not merely a function of the data itself.

These points are illustrated in Fig. 2.14, which shows the least squares AR estimation of the spectra presented in Fig. 2.7 (Marple and Lawrence, 1987). This estimate does a far superior job modeling the actual spectrum than the classical approach, but excessive peaks are evident.

\subsubsection{Autoregressive-Moving Average (ARMA) Model}

It has been mentioned above that the AR estimate is not suitable for cases with a low signal-to-noise ratio (SNR), since the all-pole model is not valid for "sinusoid in white noise." While most strong-motion records have a high SNR, an important insight can be gained by examining this problem (Kay and Marple, 1981). The white noise corrupted AR process is defined as :

$$
y_{n}=x_{n}+w_{n}
$$

where $\mathrm{x}_{\mathrm{n}} \quad=$ pure AR process

$\mathrm{w}_{\mathrm{n}} \quad=$ observed white noise with mean $=0$ and variance $\sigma_{\mathrm{w}}{ }^{2}$. 


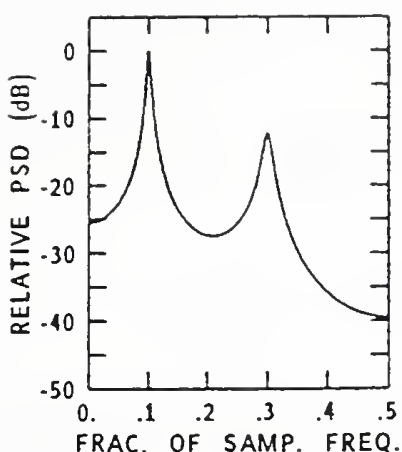

(a)

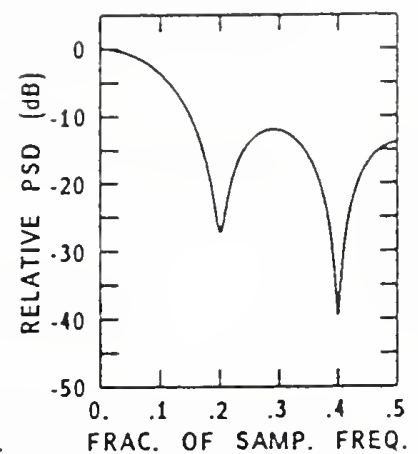

(b)

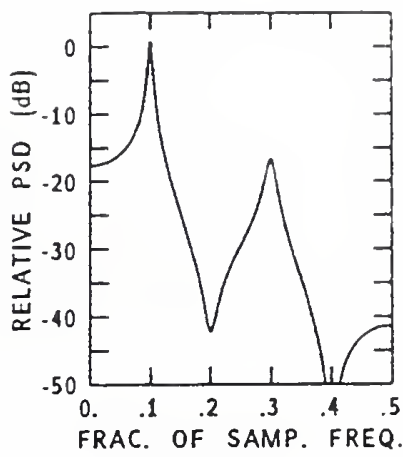

(c)

Fig. 2.13 Typical parametric spectra from the (a) AR(4) model, (2) MA(4) model, and (3) ARMA(4,4) model (Marple, 1987).

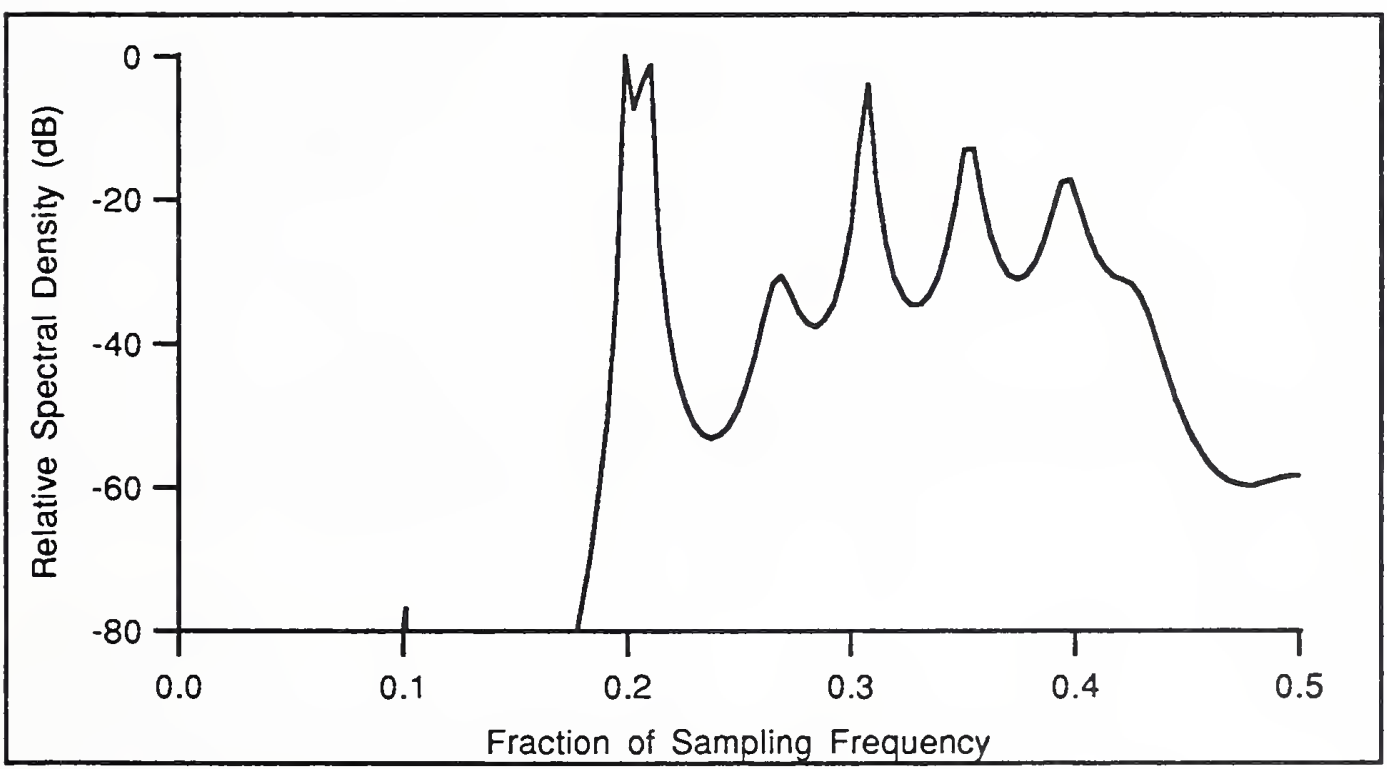

Fig. 2.14 AR spectral estimate of test series to be compared with the true spectrum in Fig. $7 \mathrm{~b}$. Note the typical "peaky" shape. 
Since the white noise is uncorrelated with the process $x_{n}$, it can be shown that

$$
P_{y}(z)=\frac{\left[\sigma^{2}+\sigma_{w}{ }^{2} A(z) A^{*}\left(\frac{1}{z^{*}}\right)\right] \Delta t}{A(z) A^{*}\left(\frac{1}{z^{*}}\right)}
$$

where $\mathrm{P}_{\mathrm{y}}(\mathrm{z}) \quad$ = spectral density of the corrupted AR process in the Z-domain

$\mathrm{A}(\mathrm{z}) \quad=$ filter parameters

* $\quad=$ complex conjugation

$\Delta \mathrm{t} \quad=$ digitization rate.

$\sigma^{2} \quad=$ variance of the output.

The noteworthy property of Eq. 2.42 is the fact that the spectral density is characterized by parameters in both the numerator and denominator. This model was described by Eq. 2.31 and is an ARMA model characterized by both poles and zeroes. The ARMA model is very powerful in that it can easily model sharp drops, sharp peaks, and smooth spectral behavior. It is also the most parsimonious estimator. Since the ARMA model is the most general, it's use would eliminate the problem of deciding whether the process is $A R$ or MA.

The major problem of implementing an ARMA model is the difficulty in calculating the parameters. The most straight-forward approach is to solve the Yule-Walker normal equations for the ARMA process. However, in this case the equations are very nonlinear and difficult to solve (Cadzow, 1982). Simply applying a least squares solution is not sufficient since there is no guarantee of convergence, or if the given answer is the result of convergence on a local extreme.

A common solution is to solve for the AR and MA parameters separately and then rationally combine the two. This is done by generating the extended Yule-Walker equations, an example of which is given in Cadzow (1982). Treitel derived a least squares technique that solves the ARMA parameters in a unified manner, has a minimum-delay denominator, and always converges (Gutowski et al., 1978; Treitel et al., 1977).

Another problem to be considered is the estimation of orders of both the AR and MA parts of the ARMA model. In addition, for the more complete system identification models, the orders of the noise system must be consistently estimated independently from the system (van den Boom and van den Enden, 1974). For some physical systems, such as the SDOF oscillator for which the AR model was derived, the estimation of AR order has physical meaning. For other processes, the order must be guessed at, with various theoretical and empirical methods of deciding the optimum AR order. Akaike (e.g. 1981, 1970) approaches the problem from a similar point of view as Burg, and uses a maximum entropy criteria for deciding the proper model order. This criteria minimizes the 
"information distance" between the model and the actual process. Too few parameters and the distance is great, too many and the distance slowly increases due to over-determination. The criterion is the so-called Akaike Information Theoretic Criterion, or AIC.

There are many empirical methods proposed to determine the proper model orders (e.g. Marple and Lawrence, 1987; Bohlin, 1984; Astrom, 1980; van den Boom and van den Enden, 1974). The most straight-forward method is to increase the orders until the "innovations" series

$$
\left(y_{t}-\hat{y}_{T}\right)^{2}
$$

where $\mathrm{y}_{\mathrm{t}} \quad=$ actual output at time $\mathrm{t}$

$\hat{\vartheta}_{\mathrm{t}}=$ prediction of output at time $\mathrm{t}$ made at time $\mathrm{t}-1$

becomes white noise. A second check that all the information is being "used" by the estimation is to check if the cross-correlation of the input series and the innovations is white (Astrom, 1980). At this point, all the information available has been used.

Through a process called Wold decomposition it can be shown that AR, MA, and ARMA processes are related, in that a ARMA or MA process of limited length is equivalent to an AR model of infinite length. An infinite length of MA filter is also equivalent to a given $\mathrm{AR}$ or $\mathrm{ARMA}$ series. Therefore, a AR model with order much greater than the true model order is a possible solution to a low SNR condition. In order to avoid the problem of spurious peaks in the spectral estimate, the model order should be kept to less than half the number of data points.

Another method for dealing with noisy data is to compensate for the uncorrelated noise. This is done by either weighting the zero lag of the autocorrelation, or a similar process with the AR coefficients. The rationale behind the increased weighting of the zero lag is that the noise is considered white, with an autocorrelation of one at the zero lag and zero thereafter. Increasing the value of the actual autocorrelation at lag zero effectively suppresses the effect of the noise on the solution.

\subsection{Spectral Estimation}

\subsubsection{Introduction}

The parametric and non-parametric methods discussed are just a few of the more popular models for describing a process. While the thrust has been towards spectral estimation, it must be remembered that all these methods are time domain solutions with spectral analogs. The important question now becomes, in the words of John Tukey, "When should which spectrum approach be used?" (Tukey, 1984). Unfortunately, there is no easy answer to this question for any interesting real-life situations. For problems of interest, such as analysis of an earthquake strong motion record, the theoretical caveats needed for the 
mathematical derivation are not applicable, in particular the assumption of stationarity.

An important argument is made by Brillinger and Tukey to approach the subject by using "leading cases" (Brillinger and Tukey, 1984). An example of this concept is the use of point mass assumptions in mechanics, which strictly is not "correct," but is commonly used without complaint and with outstanding results. Rather than approach spectral analysis with strict hypotheses, Brillinger and Tukey suggest looking at the practice of stochastic spectral estimation as "...an umbra within a penumbra."

Three successively larger spheres of application can be described:

(1) An inner core of mathematically derived application. This region is generally too narrow for application to real problems. An example is a process that actually is a sum of a few pure sinusoids, or a process with these qualities unvarying, realization after realization. No example from physical science can be given.

(2) A middle area where there is reasonable understanding of the process and the performance of the method used to assess the process; the meaning of the spectrum is clear. There usually is additional information about the process at hand, but all detailed questions are not yet able to be addressed. This large area is dominated by "stationary" processes with finite variance. "This is not because the region of use of the concept of a spectrum is confined to stationary processes, but because stationary processes are easier to think about."

(3) The third, largest, region is where the technique is actively being used by practitioners and researchers. In this area the understanding of the model and process gained in the inner two areas is applied and used. A prime example is the use of spectral techniques with non-stationary data or "unique", one-of-a-kind data from a process that "...having a process would require an ensemble of parallel worlds." (earthquakes?). The famous work of Munk on ocean swells is a perfect example of the important information that can be gained by applying spectral methods to very non-stationary time series (Munk and Snodgrass, 1957).

A "non-stationary" process, such as an earthquake strong motion record, can be interpreted in many different ways. On one end of the scale the signal can be seen to be made up of short stationary segments, each of which has a story to tell. In fact, this is a common and useful method for working with non-stationary signals. Both the Thomson and Burg estimates of spectral density were derived for use on such short data series. Another approach to the "microzonation" of a data record is the concept of evolutionary spectra put forth by Priestly (1988). In this approach, the spectrum of an oscillatory process is estimated in the "neighborhood of time instant $t$ " rather than over all time. 
The other end of the scale is to view physical processes in a very macroscopic light (Brillinger and Tukey, 1984):

All we know of the world is consistent with the idea that all events are periodic with period $10^{20}$ years. And a process made up of all displacements of a periodic phenomenon - with uniform probability - is a stationary process. Thus anything we find in the world could, with this definition, come from a stationary process.

\subsubsection{The Concept of a "Spectrum"}

Non-stationarity does not doom spectral analysis, but requires extra diligence and care to insure that meaningful estimates are given. The implications of each step must be examined so that effects such as leakage do not cover the desired information in the process. The most important implication that must be addressed is, what is meant by the concept of a "spectrum"?

As commonly used, the frequency spectrum represents a given signal in time as sinusoids of various frequencies. The various frequency components present in the signal are presented in their relative energy amplitudes. This usage implies an oscillatory signal made up of "discrete" sinusoidal components. An example might be the several modes of excitement of a simple oscillator, or the characteristic frequencies of a "good" rotating bearing verses "bad". The calculation of the spectrum is merely a mathematical transform which sometimes makes a desired facet of the data easier to find. In this sense, the spectrum is no different than the use of semi-log or square-root plots.

This concept of frequency spectrum is in direct contrast to the representation of an arbitrary waveform shape by its Fourier frequencies. A single square-wave pulse has a wellknown spectrum, the sinc function, made up of theoretically infinite frequency components. In the present context this square-wave does not have "frequency" since it is a single pulse without multiple oscillations. Multiple occurrences are needed before one can speak about the frequency of an occurrence and interpret the information yielded as the energy contained in the various frequencies of oscillation.

For practical applications even this straight-forward "definition" of the spectrum has conflicting implications. Priestly (1967) gives the example of an exponentially damped sine wave, a very common physical realization :

$$
y_{t}=e^{-\alpha t} \sin \left(2 \pi f_{0} t\right)
$$

where $\mathrm{f}_{0} \quad=\omega_{0} / 2 \pi$.

An example of this signal at $\mathrm{f}_{0}=20 \mathrm{~Hz}$ and $\alpha=8$ is given in Fig. 2.15a. The Fourier spectrum pair is shown in Fig. 2.15b, where it is seen that the spectrum, while having a peak 


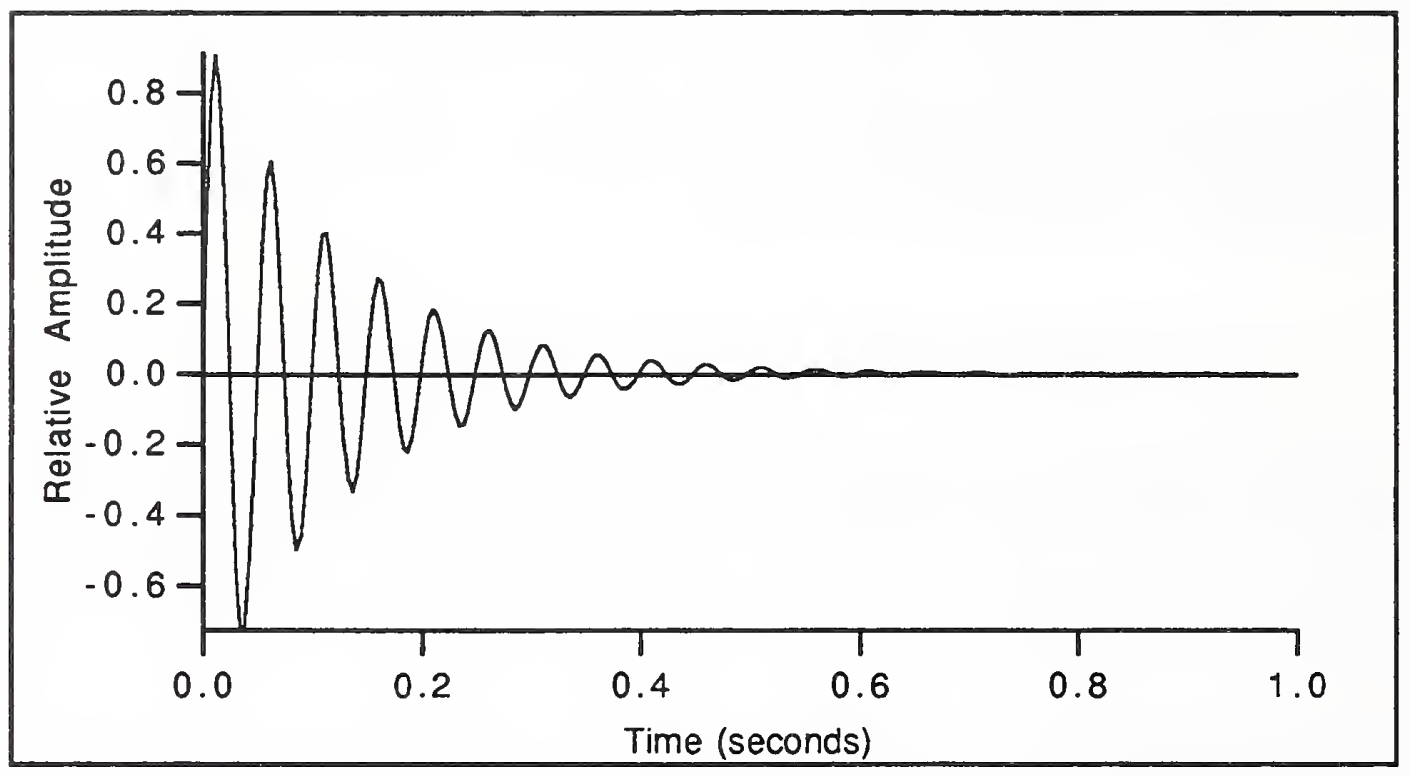

Fig. 2.15a Time history of damped sinusoid $f(t)=e^{-8 t} \sin 20(2 \pi t)$.

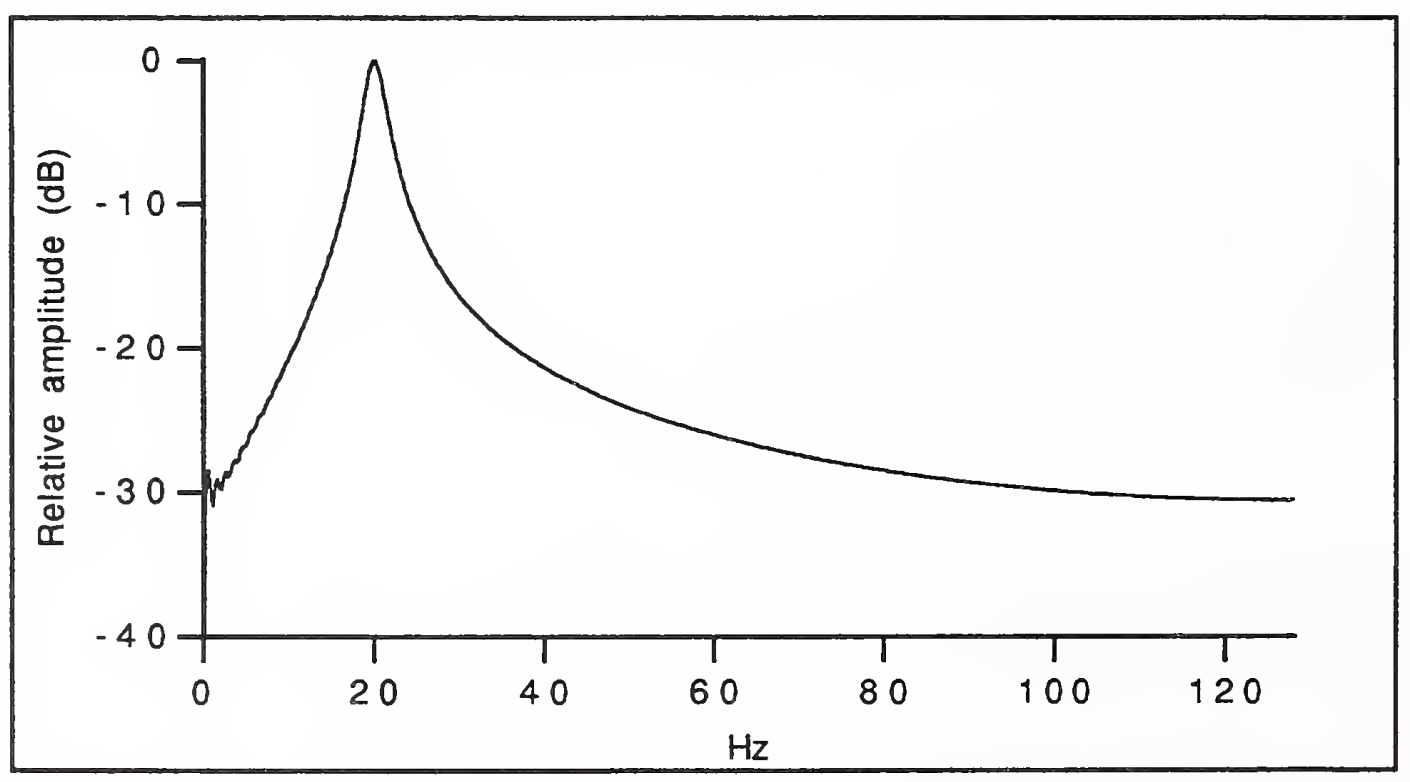

Fig. 2.15b Fourier amplitude spectrum of damped sinusoid $f(t)=e^{-8 t} \sin 20(2 \pi t)$. 
at $20 \mathrm{~Hz}$, contains components of all frequencies. This is a function of the damping, which makes the signal non-stationary since the variance is continuously changing. However, practical considerations gives the alternative interpretation that the frequency is a constant $20 \mathrm{~Hz}$ while the amplitude is changing as a function of time. No engineer with real-world experience would dispute the pronouncement that the signal in Fig. 2.15a has a spectrum indicating a single oscillatory frequency of $20 \mathrm{~Hz}$. In this case the results are "theoretically" incorrect, but for the engineer estimating the process parameters the "error" at $0 \mathrm{~Hz}$ has the benefit of yielding the system damping.

\subsubsection{Non-stationary Signals}

It is interesting to note that if an "infinite" sinusoid at $20 \mathrm{hz}$ is sampled over a finite time, and windowed to minimize leakage, results are very similar to Fig. 2.15b. In essence sampling turns a signal into a transient and windowing makes the transient non-stationary. An earthquake strong motion record becomes slightly more "proper" when it is thought of as a self-windowed signal (of infinite length if desired) with some white background noise. In this case, the effect of the self-"windowing" is useful since it can provide an insight into the system damping.

Inherent in the transformation of a time signal to the frequency domain is the averaging of the signal components over the sampling period T. A piece of time is frozen over this period and the assumption made that all time before and after is the same, i.e. repeated forever. The energies present at each component frequency are integrated over the entire time period $T$. The difficulty with non-stationary signals is that these energies are changing during this period. If the frequencies present are changing over this time window, the resulting estimation, regardless of method used, will be a smeared average as if all the frequencies with energy were active throughout the entire period.

For weakly non-stationary processes, the effect over a small time period is unimportant. If needed, the signal can be cut into relatively stationary sections and spectra found using methods specially designed for short data segments, i.e. Thomson's or Burg's method. This approach can be optimized using an Akaike AIC criterion (Akaike, 1974; Gersch and Brotherton, 1982).

If newer, recursive/adaptive methods, such as Kalman filtering, are used to yield a time-varying parameterization of the process, the idea of "frequency" must again be examined. The concept of an "instantaneous" frequency in the traditional context is meaningless. There must be some time period $\mathrm{T}$ over which to sum the energy expended (work) at each frequency. The work done is averaged over $\mathrm{T}$, hence power spectrum (power $=$ work done/time taken).

This problem is largely one of semantics arising from the imprecise manner of everyday language. In its most common, and traditionally correct usage, the spectrum 
(amplitude, energy, or power) is the infinite sum of sinusoids with arbitrary amplitude and phase, necessary to represent a given waveform. In this usage the waveform can be stationary, or a non-stationary transient, oscillatory or simple. In this usage a particular duration of signal is also not required, e.g. the Dirac delta function being represented by a sum of sinusoid of all frequencies. As discussed earlier, this approach is strictly correct only for the infinite analog case, and application to the discrete, digital domain has attendant problems.

In the case at hand, the problem is to describe or parameterize a process. In actuality, all the approaches discussed take place in the time domain (the classical Fourier approach is actually a computationally simpler version of the autocorrelation approach), yielding an impulse response function. This function is customarily transformed into the frequency domain, now called the frequency response function or transfer function, since this representation is visually less complicated and the desired system characteristics are more obvious. In particular, the system fundamental frequency is exceptionally obvious whatever method is used to estimate the process spectrum.

The Fourier-based methods attempt to characterize the process autocorrelation by assuming a uniformly spaced sinusoidal basis function with no explicit parameters involved. This method was seen to work fine if the process can accurately be modeled thusly. The parametric methods attempt to explicitly model the process through equations involving a limited number of parameters that must be experimentally discovered. These methods work well if the process can be accurately modeled by the chosen method, with the added difficulty of determining the model order. In both of these methods the process is characterized by the autocorrelation or model parameters. The spectral presentation is only an alternative method of presenting the data in a more palatable manner.

\subsubsection{The "Instantaneous" Spectrum}

Against this background it is sensible to speak of the "instantaneous spectrum," which now means a frequency domain representation of the behavior of the system at a given moment. At any given instant a filter (system) can be said to behave as, for example, an AR process of order two, with the two parameters representing a given damping and resonant frequency, free to change as the source motion changes or the structure undergoes damage. As described in Fig. 2.1, what is estimated is a filter that converts the known input into the measured output. The different methods discussed are merely different schemes for estimating that filter, which can be presented in the time domain or the frequency domain. With this clearer understanding in mind, methods allowing time varying system parameters will be discussed.

An early method of directly analyzing time varying signals was the use of complex demodulation (Brillinger, 1988; Bloomfield, 1976). For a signal with slowly changing 
amplitude and phase, complex demodulation will supply an estimate of these values. The method can be thought of as harmonic analysis in a local time span $t \pm \Delta t$. The frequency of interest, which must be identified beforehand, is isolated by narrow band-pass filtering. The signal is modulated by a complex exponential and then locally smoothed to yield the local amplitude at the frequency in question. This method, which is rather cumbersome and involves ad hoc filtering and knowledge of spectral peaks, has not been used for identification purposes and is included here for completeness.

Using traditional methods to estimate strongly non-stationary processes can yield very unsatisfactory results, as was seen above in the damped cosine example. Priestly (1967) describes early attempts to define an "instantaneous" spectral estimate, starting in 1952 (Page, 1952). Priestly puts forth an evolutionary (time dependant) power spectrum which he describes as local energy distributions over frequency. Priestly computes an evolutionary spectrum using spectral windows with various parameters, similar to the periodogram approach, and therefor suffers the same difficulties. Recursive least squares methods are now available which estimate AR or ARMA parameters describing the behavior of the process at each time step (Marple and Lawrence, 1987).

\subsection{Modeling Non-stationary Processes - Adaptive Filtering \\ 2.6.1 Introduction}

For practical stationary problems the most straightforward method is in essence least squares deconvolution, or calculation of the Wiener optimum filter (Kanasewitch, 1981; Wiener, 1964). This method assumes knowledge of the autocorrelation of the input signal and the cross-correlation of the input and output signal. The system parameters are solved for directly in the time domain and the defining response function transformed into the frequency response function. The problems involved are the estimation of the various covariance functions without an infinite length of data, and these problems have been discussed in previous sections. This method is valid if the signal or filter does not change during the period of interest and could be applied to stationary-segmented data.

The field of adaptive filtering was formed to model non-stationary processes. As the statistics of the signal change through time, the filter "adapts" to the changing variance with new parameters that reflect the structure of the system at that point. The predicted value for the next time step can be compared with the actual value, and the difference (innovations, Eq. 2.43) will give a measure of how well the filter is doing its job. The term "innovations" is used because this information is new information that can not be predicted by the model at this particular step.

Autoregressive parameters can be sequentially estimated so that the parameters are adaptive to the changing nature of the process (Marple and Lawrence, 1987). The AR parameters are updated after each data point, tracking slowly non-stationary signals. A 
forgetting factor, commonly a damped negative exponential, is used so that older data carries less and less weight. The spectral estimation can be made at any time step by evaluating the $\mathrm{AR}$ parameters around the unit circle, giving the spectral description of the behavior of the process at that time. The most effective variant of the recursive least squares algorithm was developed by Ljung (Ljung and Soderstrom, 1983; Ljung et al., 1978; Falconer and Ljung, 1978).

\subsubsection{Kalman Filters}

The most popular direct adaptive filter, or process model, is the so-called Kalman filter (Kalman, 1960; Kalman and Bucy, 1961). Sorenson (1970) points out that the Kalman approach is a direct descendant of Gauss's least squares, except now neither the signal nor the noise model must be stationary - the state may change from sample point to sample point. The solution is recursive and is presented in state-space (Soderstrom and Stoica, 1989) which uses differential equations rather than integral equations to represent the model.

Nau and Oliver (1979) state that the Kalman filter is based on a dynamic AR model defined by "two concurrent random equations of motion":

$$
x_{t}=H_{t-1}^{T} \phi_{t}+a_{t}
$$

the $A R(p)$ equations of motion, and the "motions" of the parameters,

$$
\phi_{t}=\phi_{t-1}+b_{t}
$$

where $\mathrm{p} \quad=$ number of prior observations utilized,

$\mathrm{H}_{\mathrm{t}-1} \quad=$ vector of $\mathrm{p}$ prior data observations $\mathrm{x}_{\mathrm{t}-1}, \mathrm{x}_{\mathrm{t}-2}, \ldots, \mathrm{x}_{\mathrm{t}-\mathrm{p}}$,

$\phi_{\mathrm{t}} \quad=$ vector of $\mathrm{p} \mathrm{AR}$ parameters,

$a_{\mathrm{t}} \quad=$ Gaussian white noise with 0 mean and variance $\sigma^{2}$,

$\mathrm{b}_{\mathrm{t}} \quad=$ Gaussian white noise with 0 mean and covariance matrix $\mathrm{Q}$.

Equation 2.46 estimates a value of $\phi_{t}$ comprised of $\mathbf{p}$ previous parameters, through a random walk equation. The estimate uses the weighted $\mathrm{p}$ previous data points, and yields a new observation $\mathbf{x}_{1}$ when added to a new noise value.

The problem is to use $x_{t-1}$ to filter and make good estimates of what values of $\phi_{t-1}$ occurred, to estimate future values of $\phi_{\mathrm{t}}$ from Eq. 2.46 and then use Eq. 2.45 to forecast future $x_{t}^{\prime}$ 's, repeating, as needed, the cycle of filtering and forecasting. (Nau and Oliver, 1979).

The least squares solution solves the equations so that the innovations (Eq. 2.43) - new, dynamic information that cannot be predicted - are minimized in a least squares sense each time step 
Kalman noted that all relevant information about all the previous data is contained in the posterior covariance matrix of the parameter distributions

$$
\hat{P}_{t}=\operatorname{COV}\left[\phi_{t}, \phi_{t}^{T} \mid H_{t}^{(x)}\right]
$$

where $H_{\mathrm{t}}^{(\mathrm{x})} \quad=$ entire history of the process up to and including time t.

The estimate is an explicit function of only the data history and the previous best estimate of the state, so that there is no need to use linear regression to completely re-estimate the AR parameters after each new time step.

Graupe (1989) discusses the extension of the Kalman filter to take into account colored measurement noise, rather than strictly Gaussian white noise. This is possible if the noise is still uncorrelated with the signal. This Augmented Kalman filter becomes a ARMA representation, with the colored noise represented by the MA component in a manner conceptually similar to Eq. 2.42. The theory behind the Kalman filter can be manipulated to yield the system parameters for the case where there is no a priori information about the noise, and even when there is no information about the input signal. However, these techniques seem designed for communications problems where the engineer actually has at least some additional conceptual information about the system being studied.

The so-called extended Kalman filter has been very successfully applied to non-linear estimation problems (Ljung, 1979; Astrom and Eykhoff, 1971). The manner of application is actually straight-forward. The Kalman model is constantly updating its estimation of the dynamic process by examining the innovations. The dynamics can be due to a changing input or noise process, or it can be due to the system itself changing. The effect is a linearization between single time steps, but if the system is changing slowly compared to the time step used, the linearization is "invisible" and the non-linear behavior is well modeled. 


\section{CHAPTER 3 ESTIMATION OF SOIL PARAMETERS USING SYSTEM IDENTIFICATION TECHNIQUES}

\subsection{A Framework of Understanding}

\subsubsection{Introduction}

This chapter will present some interesting and fruitful approaches to applying the system identification (SI) methods presented in the previous chapter. Given an excitation input and the system output, the goal is to identify various physical parameters that control the dynamic behavior of the soil mass. Typical parameters to be identified are soil layer resonant frequency and damping ratio. These are the obvious choices since it has been shown that the ARMA evaluation techniques were derived to model the dynamic process as SDOF oscillators. Other parameters that has been studied are the "amplification" of ground motion as the seismic energy moves from stiff bedrock to the much more compliant soil, and identification and simulation of strong ground motions.

This report will be concerned with the application of SI to the problem of identifying soil parameters. It will not attempt to cover the large field of work done in the application of SI to the soil-structure interaction problem (e.g. many papers in the journal Earthquake Engineering and Structural Dynamics; Ghanem et al., 1991). This report will present some interesting examples of the use of dynamic models to characterize in situ soil behavior.

The work to date in seismic SI can be divided into two large groups - continuous excitation and transient excitation. Examples of continuous excitation are shakers and ambient excitation. Earthquakes and seismic testing methods are examples of transient excitation. In relation to analysis methods available, these two group can each be divided into stationary and non-stationary sources. Excitation of the soil through a servo-controlled shaker is certainly a known, stationary force, so that the straight-forward methods of Bendat and Piersol (e.g. 1986) can be used to calculate soil damping and resonant frequency. The use of ambient vibrations, while often considered to be white processes, can very well be at least weakly non-stationary.

Seismic methods, such as impulse loading and use of explosives, are non-stationary but very repeatable. The repeatability of the input allows ensemble averaging, and the averaged process can be well characterized by simple Fourier analysis. Finally, earthquake excitation is obviously non-stationary and non-repeatable. It is for this situation, where the amount of information is limited, that system identification techniques are of the most use.

When analyzing the in situ soil profile, the geotechnical engineer must work with very large "structures." It is difficult to artificially excite a significant mass of the earth to levels suitable to employ system identification techniques for strain levels even approaching threshold strain. An obvious source for larger strain levels is an earthquake. In the past several years there have been a few instrumented sites where strong-motion records have 
been recorded within the soil as well as on the surface. For these cases the techniques discussed in the previous chapter can be used to calculate soil parameters, as well as to study how the parameters might change during the shaking.

\subsection{Continuous Forced Excitation of the Soil}

\subsubsection{Introduction}

The simplest method of determining the dynamic behavior of a structure is to use forced vibration. In this case the excitation is under complete control of the experimenter, and the input forcing function known. If the soil can be modeled as a one layer system for the problem at hand, then solution presented in Sect. 3.2.2 can be utilized (Richart et al., 1970). The driving function is stationary, and duplicate data can easily be collected. This makes a proper DFT-based spectral estimate very accurate. The gain factor can be plotted, the resonant frequency and soil stiffness found, and the soil damping calculated from the half-power point (Bendat and Piersol, 1980). This method can be expanded to examine higher modes of vibration.

\subsubsection{Applications to Multiple Layered Profiles}

In the more common situation the soil must be considered a layered system, with each layer having its own distinct set of descriptive parameters. This case is modeled as a series of SDOF linear oscillators. The system will be characterized as the compound filter that converts the known force input into the measured surface displacement output. The solution to this inverse problem is not necessarily unique, especially if the actual input motion is not precisely known and near-by "bedrock" motion is used (Udwadia et al., 1978).

An N-layered soil system typically can be modeled as an $\mathrm{N}$-degree-of-freedom lumped mass system, as illustrated in Fig. 3.1. If the mass distribution is known, Udwadia has derived a method whereby the damping and stiffness of all layers can be uniquely determined for forced vibration with one surface transducer (1986). The method is valid for linear response only. Since the force-time history of the exciting shaker and the system output is known, this method is fundamentally similar to the simple case just described. Udwadia notes that a good signal-to-noise ratio is an important parameter to insure low variance in the system parameter estimates.

A similar but less-controlled variant of Udwadia's method is to use background ambient vibrations as the excitation, making the potentially incorrect assumption that this excitation is gaussian white noise. An apparent application of this approach is presented by Ohmachi for a suite of locations in the San Francisco Bay area (Ohmachi et al., 1991). Three-component surface measurements were taken, three times each at the several 


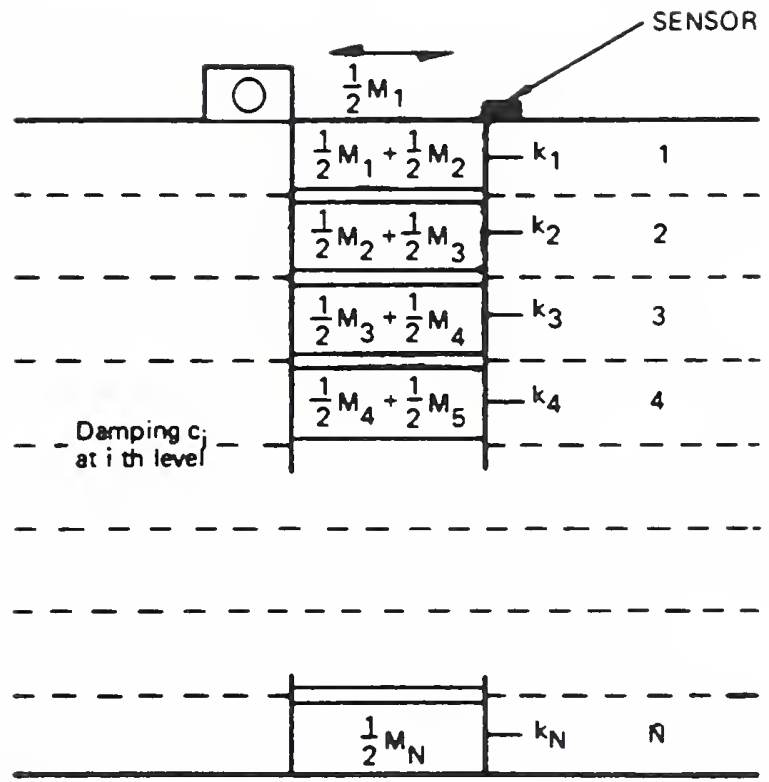

Fig. 3.1 n-Degree-of-Freedom lumped mass soil system (from Udwadia, 1978). 
locations. Small subsections (1024 points) of the data were taken, Fourier transformed, and smoothed by multiple passes of a Hanning lag window (see Newland, 1984). Three such spectra were then averaged for each component at each location.

This study was based on a method developed by the Japanese National Rail (JNR) to study soil characteristics along rail lines using microtremors (frequency content 0.5 to 20 $\mathrm{Hz}$ ) as excitation (Nakamura, 1989). Noting the virtual impossibility of taking an adequate amount of data without colored interference from social activities, Nakamura derives a method to rid the data of these effects. He claims that the social sources, the "noise", produce mostly Rayleigh waves, dominated by vertical motion. The attempt is then to eliminate the effects of the surface wave.

Based on a comparison of five rock sites and four soil sites, Nakamura claims that for rock the magnitude of vertical acceleration is the same as the horizontal. For soils the horizontal amplitude is greater than for the vertical. This is a broad assumption based on the small amount of data presented. He goes on to claim that the amplification of motion from a stiff layer to soft is through multiple reflections throughout the soil layer. This assumption is patently false since it violates conservation of energy. In fact, net energy is lost through reflection, otherwise an amplifier using no input energy could be built with a pile of plates.

This background is used by Nakamura (1989) to state that the effect of the R-wave can be measured by taking the ratio of the vertical displacement between the surface and a subsurface acceleration record. Breaking the previously made assumptions, Nakamura proceeds to derive a method that he claims will allow the estimation of the transfer function of "surface layers from tremor on the surface only." This is done through a ratio of the horizontal and vertical spectra, and called the "spectral amplification factor." The trick seems to be that, for the frequencies of interest, the R-wave has the same spectral amplitude in both the vertical and horizontal directions. He then adds, "Note, however, that the estimation accuracy drops when there exists a noise tremor agreeing with the prevailing frequency in the estimated transfer function."

While this technique would nullify common excitation factors, it would also nullify any common response characteristics. Just because a white excitation itself is uncorrelated (nonorthogonal) does not mean that the vertical and horizontal system response will be uncorrelated. Until further explanation is published, this method seems to be biased and not connected to system behavior in an unique manner. Finn (1991) states, "Nakamura's procedure for determining site periods and amplification factors is based on some tenuous assumptions." In addition, the parameters reported - "predominant frequencies" and "amplification factors" - are not the commonly understood process parameters and are index properties. 
The results presented in Nakamura (1989) shows that the method gives acceptable results for certain conditions. Indeed, JNR would not monitor the entire length of all Shinkansen lines if the method gave no useful results. Since the given derivation is contradictory, either the method works because of R-wave coincidentally canceling for the sites of interest, or there is a more complete derivation that has not been presented in English. In addition, there is a problem with using microtremors to characterize the strong motion behavior of a site. Data from Japan show that the microtremor techniques overestimate the transfer function by up to a factor of five compared to the strong motion results (Seo, 1989). In addition, evidence shows that the fundamental period shifts for strong motion accelerations.

\subsubsection{Modal Analysis}

The behavior of a well-defined structure under forced vibration can be used to ascertain information about the supporting soil system. The results of these tests have been used to measure soil stiffness beneath bridge piers (Crouse and Hushmand, 1987). Thirty accelerometers were placed at various locations of the bridge, and a detailed modal analysis of the bridge response to a large eccentric mass shaker was undertaken. It was found that the second (primary torsional) mode was found to best characterize the soil-structure interaction.

A variational form of Rayleigh's principle was used to estimate the stiffness of the Winkler springs used to model the stiffness of the soil beneath the bridge footings. Lagrange's equations were used to write the equations of motion relating to an assumed deflection shape. The actual geometric, inertial and displacement responses of the bridge were then substituted into these equations, and spring stiffnesses estimated when the substitution led to convergence.

The calculated stiffness of the sounding soil mass matched very well with results from finite element analysis and experimental estimates. The computed resonant frequencies were almost identical to those observed for the first four modes. While yielding good results, this method is quite complicated, both experimentally and computationally. In this case thirty channels of acceleration data had to be recorded and analyzed. In addition, the authors note that the technique will only work with very well characterized structures since the effects of soil and structure must be separated. The values given are for an averaged lumped soil mass and not for each soil layer independently, therefore this method would probably not be of much help to identify layers of potentially liquefiable sand.

\subsubsection{Estimation of Soil Properties from Impedance Functions}

One powerful method of describing the dynamic interaction between a vibrating rigid body and the soil is through dynamic impedance functions. The method was developed to 
analyze rigid machine foundations as a function of the excitation frequency. The dynamic impedance function, $\mathbf{K}_{\omega}$, is decided for an "associated" massless foundation, and then the steady-state behavior of the actual foundation and any mass resting on it can be easily calculated. The impedance function can be determined analytically, numerically, or experimentally. The following discussion of impedance functions is from a paper by Gazetas (1983).

For each harmonic excitation frequency the impedance, $\mathbf{K}$, is defined as the ratio of the steady-state force and resulting displacement at the interface of the soil and foundation. The vertical impedance can be written as

$$
K_{v}=\frac{R_{v} e^{i \omega t}}{V e^{i \omega t}}=\frac{\text { harmonic vertical force }}{\text { uniform harmonic settlement }}
$$

Since the dynamic force and displacement will generally be out of phase, the displacement can be split into one component which is in phase with the force, and an orthogonal component. The dynamic impedance can then be expressed in a complex form:

$$
K_{m}(\omega)=K_{1 m}(\omega)+i K_{2 m}(\omega)
$$

where $\mathrm{m} \quad=$ displacement mode, i.e. vertical, horizontal, rotational, torsional, or coupled.

In this format, the real part contains the effects of the stiffness and inertia of the supporting soil mass, and the imaginary part describes the radiational and material damping of the soil mass. Both the stiffness and material damping are believed to be frequency independent, so $\mathrm{K}_{\omega}$ will vary due to the frequency dependant behavior of inertial mass and radiational energy loss.

The SDOF oscillator can also be represented by the complex dynamic impedance function in the form of Eq. 3.2:

$$
K(\omega)=\left(k-M \omega^{2}\right)+i C \omega
$$

$$
\begin{array}{ll}
\text { where }\left(\mathrm{k}-\mathrm{M} \omega^{2}\right) & =\mathrm{K}_{1} \\
\mathrm{C} \omega & =\mathrm{K}_{2} \\
\mathrm{M} & =\text { mass } \\
\mathrm{k} & =\text { static soil stiffness } \\
\mathrm{C} & =\text { soil damping. }
\end{array}
$$

Therefore, if the dynamic impedance function for a specific footing and soil system can be both experimentally measured and numerically calculated, the soil parameters can be identified through an iterative process. 
The impedance function, Eq. 3.3, can be written in a more common form:

$$
K=k\left\{\left(1-\frac{\omega^{2}}{\omega_{n}^{2}}\right)+i 2 \beta \frac{\omega}{\omega_{n}}\right\}
$$

where $\mathrm{k} \quad=$ static soil stiffness

$\beta=\mathrm{C} / \mathrm{C}_{\mathrm{cr}}=\mathrm{C} \omega_{\mathrm{n}} / 2 \mathrm{k}=$ damping ratio

$\omega_{\mathrm{n}} \quad=\sqrt{ }(\mathrm{k} / \mathrm{M})=$ natural frequency

or, in short-hand form,

$$
K=k\left\{\kappa+i \omega c_{s}\right\}
$$

where $\mathrm{k} \quad=$ static soil stiffness

$$
\begin{array}{lll}
\kappa & =\left(1-\omega^{2} / \omega_{\mathrm{n}}^{2}\right) & =\text { stiffness coefficients } \\
\mathrm{c}_{\mathrm{s}} & =\mathrm{C} / \mathrm{k} . & =\text { damping coefficients. }
\end{array}
$$

Gazetas notes that, in the form of Eq. 3.5, the dynamic impedance of the SDOF oscillator can be seen as the product of the static stiffness of the soil and a complex number incorporating the dynamic characteristics of the system. The stiffness coefficients decrease with increasing frequency while the damping coefficients stay constant, as shown by the chart in Fig. 3.2.

In reality, however, the relation between the stiffness and damping coefficients, and frequency, is complex for soil-foundation systems. The behavior of the dynamic factor of the impedance function will depend on the mode of vibration, geometry, rigidity, and embedment of the foundation, and the profile and elastic properties of the soil system. These complications are the reason that difficult numerical techniques, only available since the mid-1970's, must be used to estimate impedance functions. The experimental determination of these functions has also been a difficult task (Crouse et al., 1990).

A small rigid footing can be excited by forced vibrations to determine actual impedance functions in situ (Luco and Wong, 1990). The soil is modeled as several horizontal layers overlying a half-space. The parameters of the theoretical function are varied until there is a least squares match between it and the experimental function. This allows the identification of the shear-wave velocities (stiffness), material damping ratios, and Poisson's ratios of the soil layers. Luco and Wong used the full set of impedance functions, incorporating all five modes of vibration, in their theoretical calculations. 

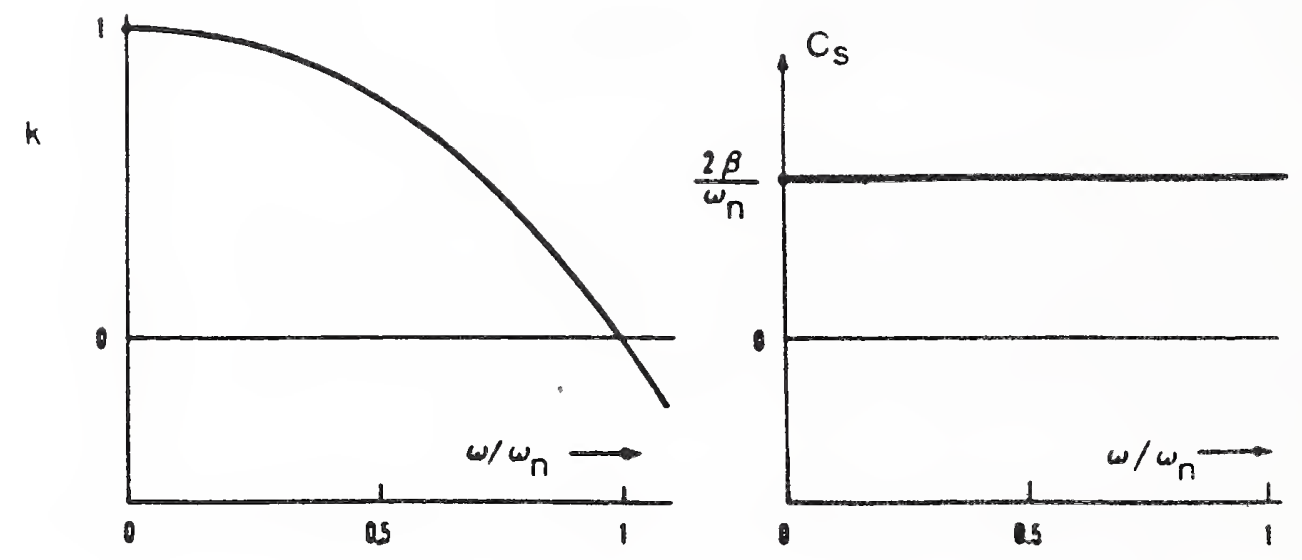

Fig. 3.2 Dynamic stiffness and damping coefficients of a SDOF oscillator (from Gazetas, 1983) 
This technique was evaluated with a set of data from a foundation test in the Imperial Valley, CA (Crouse et al., 1990), which only contained the experimental functions for three modes. The resultant velocities were different from the experimentally measured values, but within the experimental variance of the SASW method used to measure the in situ velocities. However, the damping ratio values were very erratic and unrealistic.

The impedance function method has been further refined to improve the damping estimates (Luco and Wong, 1992). The primary concern of this paper was to ascertain the effect of frequency on the identification accuracy. Since the applied force is proportional to the square of the frequency, the force at low frequencies is small and approaches the noise level. However, damping values are best defined at low frequencies. It was shown that damping ratios for soil deeper than one-quarter of the wavelength associated with the frequency of interest cannot be accurately identified. A similar relationship between wavelength/frequency and depth of sampling was found in the derivation of the SASW method.

Luco and Wong (1992) also found that data error degrades the estimates of the properties of deeper layers, especially the damping ratio. As expected, the use of more impedance functions (different modes) improves the identification. The authors improved their results by the use of a weighting function (smoothing) on successive estimate iterations.

While the use of impedance functions to estimate in situ soil properties shows promise and has good theoretical grounding, it is very limited as to the depth of soil it can characterize. The maximum is only a few meters. The experimental and analytic overhead is also quite daunting. The forced vibration techniques differ from the typical system identification methods discussed in Chapter 2 in that the parameters were identified through mechanistic/deterministic models of specific behavior rather than fitting the output time series to a more general model such as the AR feedback model.

\subsection{Non-stationary Excitation of the Soil System}

\subsubsection{Continuous Excitation}

Forced vibration methods are often limited because there is no way to input enough energy into the system to allow a large amount of soil to become involved. A solution to this problem is to use natural excitation. A constant, repeatable source would be appropriate, since it would allow large data sets and redundancy to minimize bias and variance in the estimates. Ideally, simple DFT methods could be used with excellent results. Of course, this assumes that the natural signal is stationary, as was assumed by Ohmachi et al. (1991). For example, Gersch et al. (1973) used a maximum likelihood ARMA estimation of the system parameters from two different length time series from a wind-loaded building. The estimated damping ratio and resonant frequency, and their coefficients of variation, were consistent for the first two modes of the 625 point and 2500 point series. This led the authors to conclude that, in this case, the wind loading is a white noise excitation. 
Ambient vibration, however, is non-stationary in the long-term. A straight-forward solution is to break up the non-stationary record into stationary pieces. The simplest method is to assume that the variance is changing slowly, and break the record into relatively short, equal-length segments. These segments can then be independently analyzed and modeled (Popescu and Demetriu, 1990; Gersch and Martinelli, 1979).

A much more efficient approach is to use the Akaike Information-theoretic Criterion (AIC) to divide the record into locally stationary, contiguous but not necessarily equal length segments (Gersch and Brotherton, 1982). Each locally stationary segment is then assumed to be made up of two components. The first is a stationary time series that is the same for all the segments and represents the time invariant parameters of the structure being investigated. The second is a non-specific auxiliary signal and varies from segment to segment, and can be due to various noise sources.

While it was shown that a regularly sampled vibration record is exactly characterized by a $2 \mathrm{n}-2 \mathrm{n}$ order ARMA model (Gersch et al., 1973), Gersch simplifies the problem by noting that the MA parameters contribute very little to the estimates of the structural parameters. As explained in Chapter 2, the problem is greatly simplified by modeling the process as strictly $\mathrm{AR}$, since the calculation procedure is well characterized. It was stated earlier that MA parameters provide information on the phase relation of the time series, while the values of the natural frequency and damping ratio depend only on the AR parameters (Chang et al., 1982).

The model used, with an auxiliary process, is the ARX model. The AR spectral estimate is calculated from the AR parameters, and a corresponding pole plot made for each optimal time segment. Since the structural part is common to all segments and the auxiliary (noise) part different in each, the complex poles of the structural part will be in the same location while those of the noise process will vary in each pole plot. This is how the parameters of interest are pulled out of the general model.

This ingenious method allows more certain estimates of the damping parameter. Since the variance of the damping estimate is a function of the inverse of the length of data used to make the estimate, long stretches of stationary data are usually required for robust estimates. The present method allows the estimates from each segment to be combined with a final variance inversely proportional to the length of the sum of segments.

Gersch and Brotherton (1982) present an example based on ambient vibration taken from the top story of the Jet Propulsion Laboratory at the California Institute of Technology. The values for natural frequency and damping ratio for the first two modes are given in Table 3.1. These values are very realistic, and the variance of the damping is two to four times smaller than an earlier analysis made with arbitrary, constant length segmentation. 
Table 3.1 Estimates of Natural Frequency and damping Ratio for the JPL.

\begin{tabular}{l|c|c} 
& Natural Frequency (std. dev.) & Damping Ratio (std. dev.) \\
\hline First Mode & $1.02 \mathrm{~Hz}(0.007 \mathrm{~Hz})$ & $2.8 \%(0.57 \%)$ \\
\hline Second Mode & $3.14 \mathrm{~Hz}(0.02 \mathrm{~Hz})$ & $3.4 \%(0.35 \%)$ \\
\hline
\end{tabular}

\subsubsection{Modeling of Earthquake Strong Motion}

Earthquakes can be seen as high-energy forcing functions that can be used to excite a system of interest. Since the earthquake is a one-time transient event, it is by definition non-stationary. It also is an unique event, so averaging techniques available to continuous excitations are not applicable. Use of parametric models have the advantage of being stochastic in nature, parsimonious, and directly related to structural properties. If parametric models can accurately simulate a process, then the necessary filter has been discovered and the parameters can be used to identify structural properties. The same reasoning is true if the one-step predictor can be estimated with a parametric model. Up to this point, the assumption has been made that earthquake strong motion can be accurately modeled by AR or ARMA techniques without thoroughly investigating whether this is so. This important assumption will now be examined.

In the 1960's and 1970's there was great interest in modeling seismic events for the purpose of identifying the source. The driving need was the ability to differentiate nuclear explosions from seismic events. It was assumed that there is a difference between the ground motions due to earthquakes and explosions. If earthquakes and explosions could each be well represented by an AR or ARMA model, the essence of each process would be condensed into a few parameters, greatly simplifying the discrimination task. In addition, for certain model structures, the parameters are related to important physical properties such as earthquake intensity, duration, and distance (Cakmak and Sheriff, 1984).

A detailed attempt to discriminate between blasts and earthquakes derives an AR model from the initial displacement potential of the two source mechanisms (DargahiNoubary et al, 1978). The P-wave history was found to be modeled as a third-order AR process. The parameters identified by this analysis are useful for estimating source properties, such as event duration, and reflections of the direct P-wave. While these parameters are not of much use to ascertaining the near-surface soil properties, they are of use to the reflection seismologist.

Closer to the problem at hand, a very understandable exposition of the segment approach and the application of the Burg estimator of AR parameters to earthquake characterization is given in a paper by Jurkevics and Ulrych (1978). In this paper the authors were interested in studying how the process changed through time, rather than assuming that the system parameters were time-invariant. The Orion Boulevard recording 
of the 1971 San Fernando earthquake was used as the data series, and the surface accelerogram modeled as a second-order AR process by the Burg method.

Three pieces of information are gleaned from the estimation - the two AR parameters and the innovations variance. For the frequencies of interest, the system only exhibited first mode response. The authors point out that while a larger AR order could have been used to model the event, using the lowest order that adequately represents the process allows "the motion to be less deterministic in nature, since the filter length is shorter." The strong motion record is cut into very short segments (one second, fifty data points) since a short segment best preserves the assumption that the process is stationary within that segment. The use of the Burg algorithm allows a good estimation to be made from fifty points (see Chapter 2).

The Z-polynomial representing the second-order system (Eq. 2.38)

$$
H(z)=\left(1-a_{1} z-a_{2} z^{2}\right)
$$

is solved by Jurkevics and Ulrych for the complex roots $z_{1}$ and $z_{b}$ which are complex conjugates located just outside the unit circle. This conjugate pair is illustrated in Fig. 3.3, in rectangular coordinates. Also shown are the values given as polar coordinates $\mathbf{R}$ and $\boldsymbol{\theta}$ $(0 \leq \theta \leq 1 /(2 \Delta t) \mathrm{Hz})$. $\theta$ gives the frequency location of the pole, or modal peak, while $\mathbf{R}$ gives the peakedness or half-width of the curve. The variance of the innovations is the amplitude scaling factor.

In order to smooth the data, deterministic curves were fitted to the plotted discrete values of the three parameters. The sharpness of the peak, $R$, changed somewhat through the earthquake process, while the frequency shift was never more than about eight percent. The innovation variance traced the envelope shape of the accelerogram through time. The study is quite successful in characterizing the time varying nature of earthquake strong motion. This helps show the validity of modeling strong motion with parametric models.

An approach more useful for identifying soil parameters is a derivation of an ARMA model for a SDOF oscillator based on an analog to the continuous-time differential equations of motion (Chang et al., 1982). The intermediary between the continuous domain and the discrete domain is the assumption of a match between actual and calculated autocorrelations. Equations are provided to calculate the damping ratio and resonant frequency of $n$-degree-of-freedom oscillators from the $2 \mathrm{n}$ AR parameters. Phase relations are preserved in the MA parameters.

Chang et al. used the Box-Jenkins estimation procedure to calculate the ARMA parameters (Box and Jenkins, 1976). The quality of the estimation is decided by how well the residuals approach white noise, and is given by the "Q" statistic (Chang et al., 1982). The Box-Jenkins method is only applicable to stationary time series, so a problem exists in applying it to earthquake records. The adopted solution was to divide the complete record 


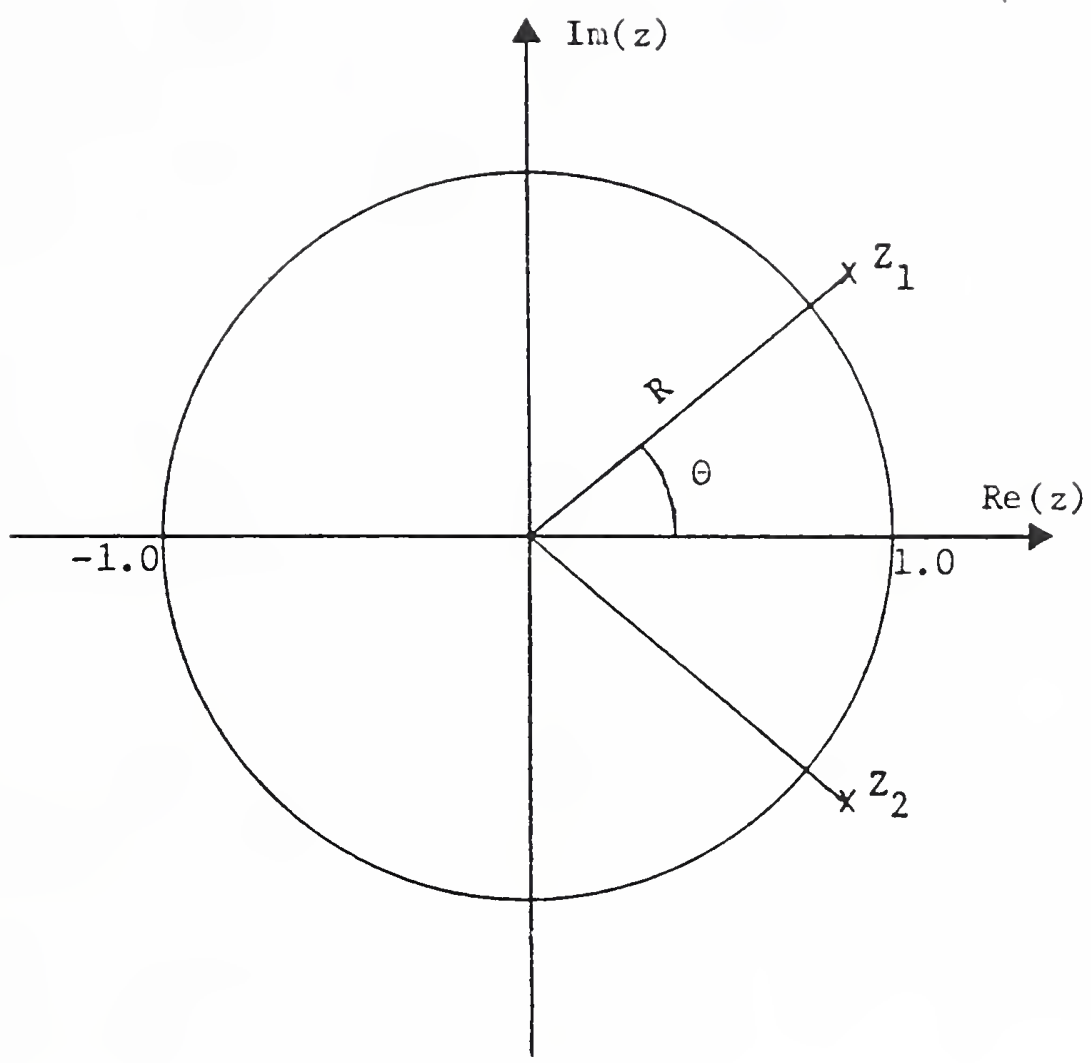

Fig. 3.3 Locations of filter poles $z_{1}$ and $z_{1}$ in the $Z$ plane, relative to the unit circle (from Chang et al., 1982). 
into constant-length quasi-stationary segments, with five second segments (250 data points each) giving the most satisfactory results.

Strong motion records from 1942 El Centro, and the 1971 San Fernando earthquakes were analyzed. Almost all the data segments were best modeled as ARMA(4,1) processes. The remaining segments were best fit by an $\operatorname{ARMA}(2,1)$ model. For the SW component of the 1940 Imperial Valley earthquake, recorded at El Centro, and modeled as $\operatorname{ARMA}(2,1)$, the parameters corresponded to the resonant frequency ranging from 2.4 to $3.2 \mathrm{~Hz}$. The corresponding damping ratio ranged from $27 \%$ to $60 \%$. However, it is not clear to what physical entity these parameters correspond to since there is not a well-defined system - a known input into a column of soil (filter) and a measured output. The confusion as to what physical quantity is being modeled is the inverse of that discussed in Chapter 2. For the case under discussion, it appears that the AR coefficients model the shape of the ground motion history, and the corresponding spectral estimate is descriptive of the waveform shape rather than the mechanical behavior of the earth system.

The Box-Jenkins method used by Chang et al. selects the model order based on the data itself, rather than by a priori decision. The authors therefore expect that other earthquakes might yield ARMA orders different from $(4,1)$ and $(2,1)$. The authors also believe that the source of non-stationarity in the surface strong motions is a result of changes in the input driving function rather than in the soil (filter), a conclusion with which a seismologist would heartily agree.

Segmentation of non-stationary strong motion records is still in use today. This approach offers a straight-forward way to apply familiar, tractable methods to a strictly inapplicable problem. A recent application applied the AIC-based segmentation scheme used by Gersch and Brotherton (1982) to earthquake records from Romania (Popescu and Demetriu, 1990). This choice was made after a thorough review of the various methods available, including Kalman filter methods that are designed for non-stationary data. An ARMA model is fitted using the Maximum Likelihood method with the goal of characterization and simulation of earthquakes with few parameters.

A preliminary canonical correlation analysis provides an "good" initial guess as to the ARMA orders, and the final choice is made from statistics developed during the Maximum Likelihood determination and application of the AIC. For simulation, each segment can be calculated by passing white noise with variance equal to that of the associated ARMA model through the calculated process filter, and then multiplying the section with an "envelope" function taken from the actual intensity of the associated earthquake segment.

In general, the simulations of Popescu and Demetriu matched the actual motions very well, with statistics such as cumulative energy and root mean square acceleration tracking throughout the record. However, there were some problems with the match for short term energy for two directional components. Short-term spectra were also calculated for each quasi-stationary segment, giving a graphical image of the change in the shaking process 
through time. This paper illustrates the utility of the segmentation method of approaching non-stationary signals, and shows that earthquake strong motion can be well modeled by the ARMA scheme.

\subsubsection{Transforming Non-stationary Signals into Stationary Signals}

Another approach to analyzing non-stationary data using methods strictly stationary in derivation, is to transform the non-stationary data series into a stationary record. The oldest and simplest technique is to apply the difference operator to the data. Often times the new data series will be stationary and the parameters solved for with customary techniques. The parameters can be adjusted to take into account the effect of the differencing transformation (Bendat and Piersol, 1986).

A more advanced approach is based on the same assumption made by Gersch and Brotherton (1982), that the non-stationary signal is a linear combination of two components; (1) a stationary component that is a result of the response of the structure in question, since the physical properties are generally thought to be time invariant (a problem in soils for strains greater than the threshold strain), and (2) the second component is a non-stationary auxiliary input to time varying noise processes.

Based on the loose definition of stationarity used in this report (data variance is constant through time), this approach sees the earthquake strong motion as a constant-mean process acted on by an "envelope" function having time-varying amplitude. Polhemus and Cakmak (1981) construct a polynomial to describe the change in variance over time and employ it to "correct" the variance to a constant. They use this method to simulate earthquake strong-motion using an ARMA model (1981). A particular earthquake is seen as an instance of a stochastic process. Therefore, a "similar" earthquake would not have to be identical, but only need to have the same stochastic descriptors. Indeed, this is the stochastic foundation that makes the AR and ARMA methods possible.

Polhemus and Cakmak (1981) devised a three-stage procedure to estimate constant parameters from a time series with a time-varying variance:

(1) An estimate of the variance function $\sigma_{z}^{2}(t)$ is made in a non-biased manner. A "power transformation" is used and fitted to a polynomial using least squares.

(2) The acceleration series is converted to a stationary series. The standard deviation at each time step is derived from step (1) and is used to normalize the non-stationary accelerations history into a constant-variance data series.

(3) An ARMA model is fit to the transformed data and the constant process parameters estimated. 
A new, statistically equivalent, earthquake record can be simulated by passing white noise through the derived ARMA model and then "denormalizing" the signal with the stepby-step standard deviations calculated in step (2). The model is validated by examination of the residuals of the actual and simulated data. If the residuals from the two are uncorrelated (white), then the model has accurately represented the process.

This method has been applied to a suite of earthquake records in an attempt to estimate seismological variables (Cakmak and Sherif, 1984). The model parameters are used to estimate values for temblor intensity, duration, distance from the site to the fault, and local geology. Statistical methods are employed to fit these variables to model parameters, which now include parameters describing the variance function $\sigma_{\mathbf{z}}{ }^{2}(t)$ as well as the familiar ARMA parameters. The brief results for the 1971 San Fernando earthquake are positive, but the authors note that much more work needs to be done before the assumptions can be accepted.

\subsubsection{Modeling of Non-stationary Processes - Time Adaptive filtering}

A non-stationary process can be modeled directly using time adaptive filtering, where the estimates of the AR parameters are updated for each time step. An early implementation of this approach to earthquake strong motion data was in the paper by Jurkevics and Ulrych (1978) discussed above. The authors modified the Least Mean Squares algorithm (Alexander, 1986) to operate on the data forward and backward, after Burg (1975). This modification results in instantaneous spectral estimates with shapes similar to that calculated by applying the Burg method to segmented data (see Sect. 3.3.2). In addition, the step size of the adaptive algorithm changed as a function of the local input amplitude.

The modified algorithm of Jurkevics and Ulrych allows the filter to be applied to the data in reverse, in order to eliminate startup error. Startup error due to poor initial estimates and a small past history is especially difficult with seismic signals which are minimum phase, i.e. energy density is concentrated at the beginning of the signal. The instantaneous estimates of the two AR parameters and the innovations variance were averaged in fifty data point segments to smooth the noise, and deterministic curves fitted to the data. The proper time step to use in the adaptive algorithm was somewhat troublesome to determine, an improper value either critically underdamps or overdamps the response.

The adaptive estimations reported by Jurkevics and Ulrych were virtually identical to those calculated by the segmentation method. This result shows that carefully segmenting non-stationary data does a very good job of characterizing the local, quasi-stationary process. The frequency and amplitude content of the accelerograms simulated by the resulting filters were the same as the actual recorded signal. 
A much more direct approach is to utilize the Kalman filter estimation of the AR parameters. Kitagawa extended the work of Akaike through a state-space AR model with constrained time-varying coefficients (1983). The model assumes a zero mean, nonstationary data series, such as an accelerogram. In contrast to the method of Jurkevics and Ulrych discussed above, where the AR coefficients could change abruptly through time and then had to be smoothed and filtered by an ad hoc technique, Kitagawa employs a linear constraint to insure that the AR coefficients can only change gradually through time.

The time varying AR model is defined as

$$
y_{n}=\sum_{i=1}^{m} a(i, n) z(n-i)+\epsilon(n)
$$

where $\mathrm{m} \quad=$ counter for $\mathrm{AR}$ order $1 . . \mathrm{k}$

$\mathrm{n} \quad=$ time step $1 . . \mathrm{N}$ number of data points.

where the AR coefficient is a gradual function of the time step counter $\mathbf{n}$. A simple least squares fit would entail the calculation of $\mathrm{mN}$ parameters from $\mathrm{N}$ data points. This large overparameterization leads to poor estimates. A parsimonious approach is to treat the AR coefficients as random variables and apply a stochastic rule as to their behavior through time. The change in the AR parameters is constrained by a "stochastically perturbed difference equation", which must reflect the actual behavior of the process being modeled. The estimation is now a constrained least squares problem.

For the simplest case, the parameters are modeled as randomly changing, modeled by a random walk prior distribution, and neighboring coefficients are almost equal. The equation governing the change is a first order difference model

$$
a(i, n)=a(i, n-1)+\delta(i, n)
$$

where $\delta=$ Gaussian white noise with 0 mean, variance $\tau^{2}$

$\mathrm{n} \quad=$ data point number (time step).

If the process, hence AR parameters, changes more slowly, the constraint is better defined by a second order difference model

$$
a(i, n)=2 a(i, n-1)-a(i, n-2)+\delta(i, n) .
$$

The order, $\mathbf{k}$, of the difference equation that best models how rapidly the system is changing through time, must be estimated, and are parameters of the model along with the AR order $\mathrm{m}$ and innovations variance. The variance of the constraint innovations, $\tau^{2}$, controls the "intensity" of the stochastic change, and must also be estimated. 
There are now $(\mathbf{m}+\mathbf{k}+2)$ parameters to be estimated $-\mathbf{k}$ AR parameters, $\mathbf{m}$ constraints, and the two variances, $\sigma^{2}$ and $\tau^{2}$, which can be combined into the ratio $\mu=$ $\tau^{2} / \sigma^{2}$. The ratio $\mu$ represents the trade-off between the (in)fidelity to the actual data and the (in)fidelity to the change constraint. The trade-off ratio has been likened to a signal-tonoise ratio, or in Bayesian terms a measure of belief in the a priori assumptions. Non-linear maximization of the likelihood parameter is used to estimate $\mu$, and the AIC is used to define the system parameters $\mathbf{m}$, and $\mathbf{k}$. At this point Kalman filtering is used to estimate the values of the model parameters.

An instantaneous power spectral density can be generated from the "instantaneous" $\mathrm{AR}$ estimates. Given the local AR parameters just estimated, $\mathrm{a}(\mathrm{j}, \mathrm{n})$ and $\sigma^{2}$, the spectral estimate can be defined by

$$
S(\omega, n)=\frac{\sigma^{2}(n)}{\left|1-\sum_{j=1}^{m} a(j, n) e^{-i j \omega}\right|^{2}}
$$

$$
\begin{aligned}
\text { where } \mathrm{i} & =\sqrt{ }-1 \\
\mathrm{~m} & =\mathrm{AR} \text { order } \\
\mathrm{n} & =\text { time step of interest } \\
\omega & =\text { radial frequency } \\
\mathrm{j} & =\text { counter for } 1 . . \mathrm{m} \text { AR order }
\end{aligned}
$$

This spectrum is simply a helpful visual aid as to the behavior of the process at that point in time.

This entire scheme was tested by comparing the theoretical and estimated instantaneous spectra for synthesized non-stationary AR processes. An example slowlychanging time series is shown in Fig. 3.4. The actual gradually changing spectrum for this data is shown in Fig. 3.5, and should be compared to the estimated spectrum shown in Fig. 3.6. The estimated spectra, for AR order 5 , second order difference constraint, and tradeoff ratio $9.6 \times 10^{-5}$, is virtually the same as the theoretical solution. The match for rapidly and instantaneously changing processes match almost as well.

An earlier paper by Kozin (1977) presented a very similar approach. However, Kozin used an orthogonal family of Legendre polynomials to "constrain" the changing AR coefficients. He also used a consistency method to apply maximum likelihood to nonstationary signals and AIC to determine the best choice for model order.

The use of Kalman filtering is presented in more detail in later papers, where the method is directly applied to characterizing earthquake strong motion records (Kitagawa and Gersch, 1985; Gersch and Kitagawa, 1985). The authors point out that earthquake 
acceleration history is modulated by a slowly changing envelope function, as described by Polhemus and Cakmak above. This envelope is the change in scaling of the AR parameters through time, or the "smoothed value of the instantaneous variance." The Kalman-Akaike based technique just discussed (Kitagawa, 1983) can be used to estimate the smoothed trend of the innovations variance, or changing power.

The "smoothness priors time-varying AR coefficient" model was applied to the 1971 San Fernando earthquake (Gersch and Kitagawa, 1985) and the 1949 Olympia, Washington earthquake (Kitagawa and Gersch, 1985). The results for the Olympia temblor will be used as an example. The authors recalculated and optimized model and constraint order every one-hundred data points, out of a total twelve-hundred. The actual accelerogram is shown in Fig. 3.7, with a simulated history shown in Fig. 3.8 for comparison.

The change of the system through time is given by the change in AR coefficients through time, shown in Fig. 3.9, and the innovations variance history shown in Fig. 3.10. Notice that the system descriptors change quite infrequently, and with "quantum" jumps. The variance envelope, or power, shows several "bumps" which the authors believe are indicative of the arrival of the various wave modes. The local impulse response function of the process can be computed from the instantaneous AR coefficients and innovations variance. This information is combined in Fig. 3.11a, which shows the instantaneous spectral description of the system through time. The spectra actually reveal no more information than the other plots, but presents the physical relationships in a more familiar manner. For comparison, Fig. 3.11b shows the instantaneous spectrum for the simulated time history, shown in Fig. 3.8.

At this writing, these methods have only been applied to model the strong motion response. It is hoped that Gersch and Kitagawa will adapt this method to estimating the parameters of a single input-single output system. Attempts are currently being made to apply extended Kalman filters to directly identifying nonlinear soil properties (Lin, 1990). This preliminary study was made for a simulated soil system for which the nonlinearity was assumed to be purely hysteretic. The input and output signals for the soil system are required for parameter identification, and estimates of the statistical nature of the noise.

The results were varied, with fair success when the model-generated backbone curves were the desired output. If the soil system is well characterized by the chosen model, then only one input-output data series is needed to completely describe the soil's nonlinear behavior. If not, strong motion records for several different strain levels are needed. At this point many assumptions were made and the system limited, but the results were heartening and further work on these lines is warranted. 


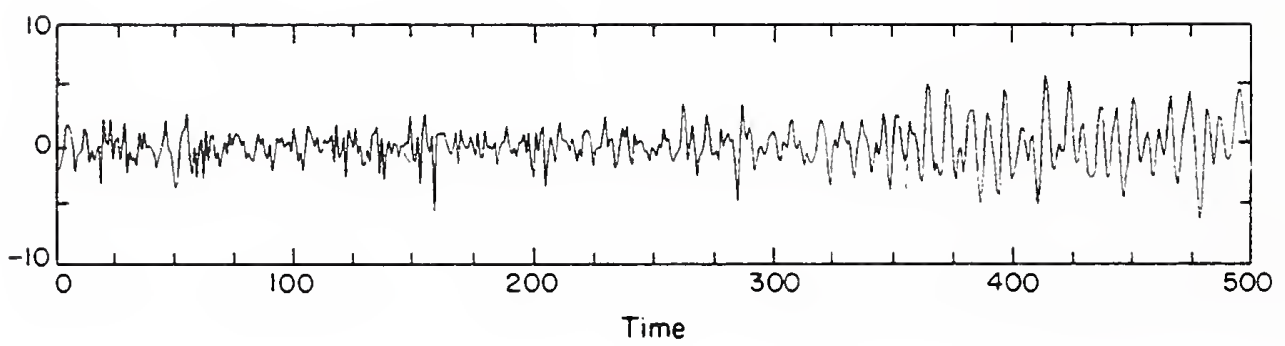

Fig. 3.4 An artificially generated AR process with a gradually changing spectrum (from Kitagawa, 1983)

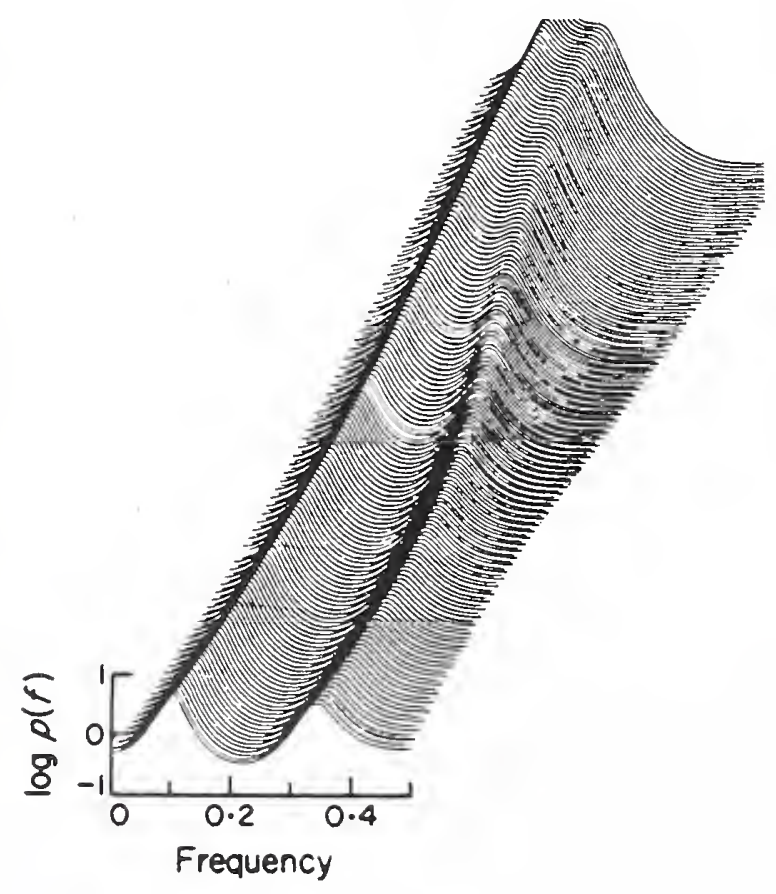

Fig. 3.5 Actual instantaneous spectra for time series shown in Fig. 3.4 (from Kitagawa, 1983).

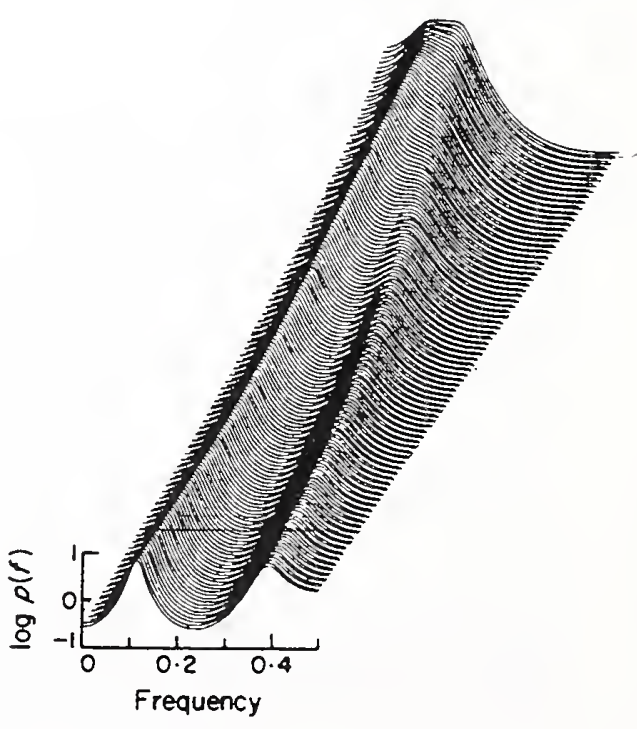

Fig. 3.6 Calculated instantaneous spectra for time series shown in Fig. 3.4 (from Kitagawa, 1983). 


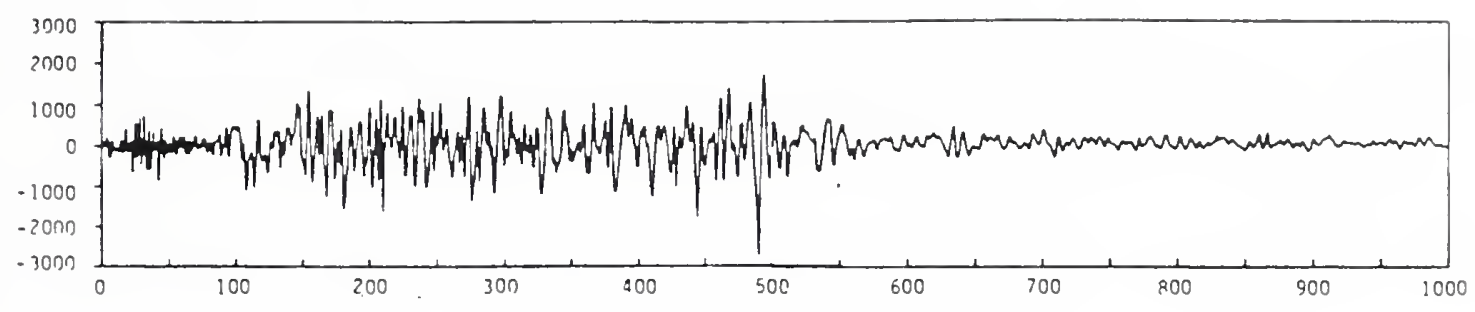

Fig. 3.7 Actual accelerogram from the 1949 Olympia, Washington earthquake (from Kitagawa and Gersch, 1985).

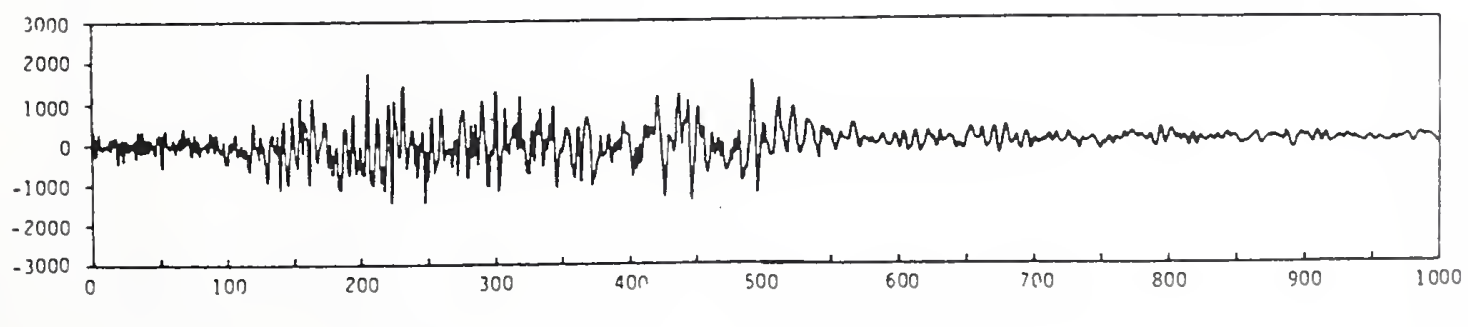

Fig. 3.8 Simulated time history for the 1949 Olympia, Washington earthquake (from Kitagawa and Gersch, 1985). 


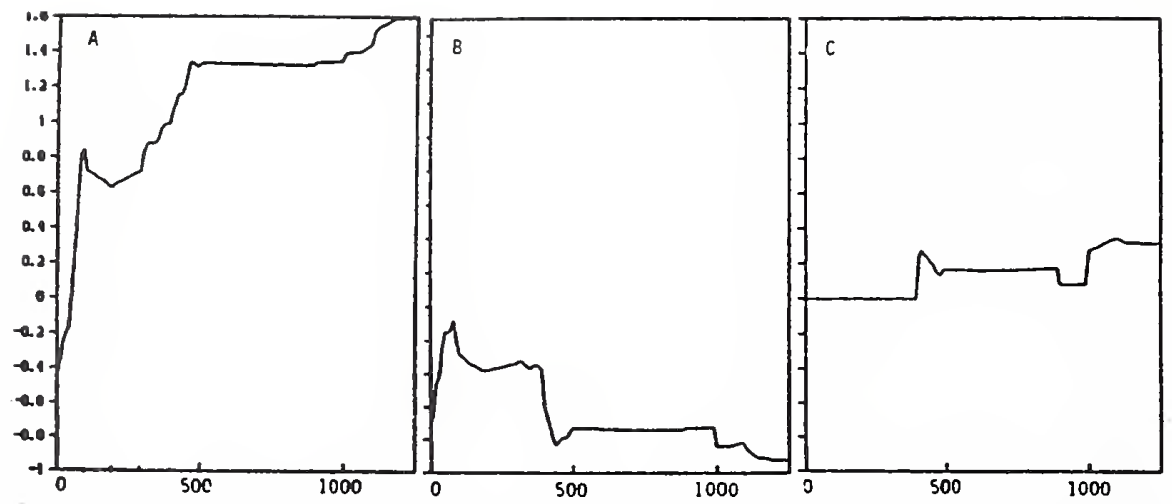

Fig. 3.9 Time varying AR model coefficients (A) a(1,1), (B) a(2,1), (C) a(3,1) for the 1949 Olympia temblor (from Kitagawa and Gersch, 1985).

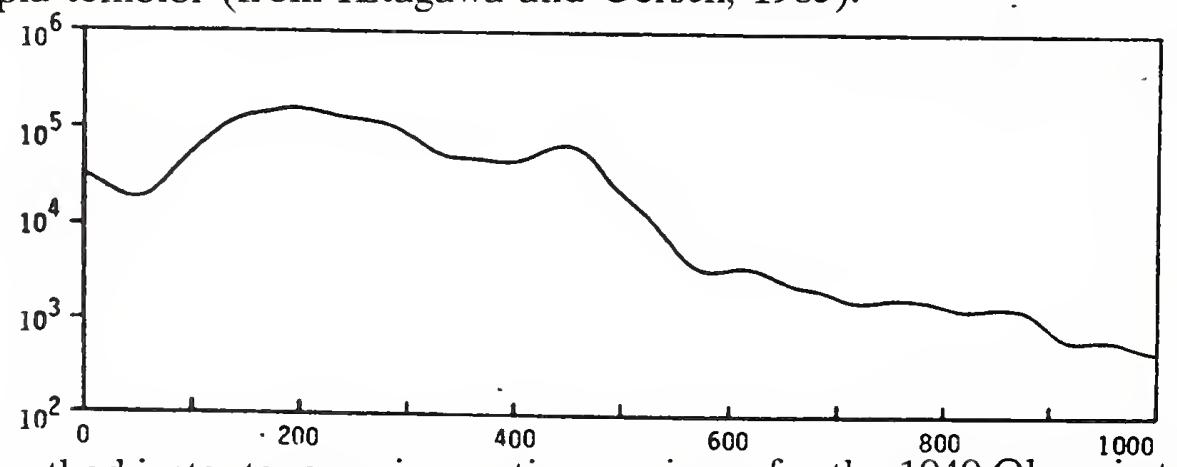

Fig. 3.10 Smoothed instantaneous innovations variance for the 1949 Olympia temblor (from Kitagawa and Gersch, 1985).

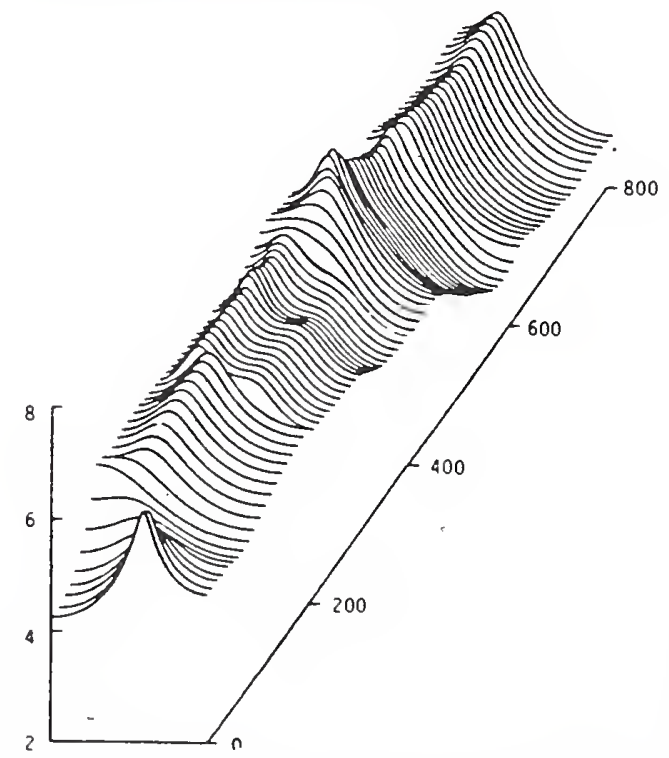

Fig. 3.11a Actual instantaneous spectrum for 1949 Olympia earthquake (from Kitagawa and Gersch, 1985).

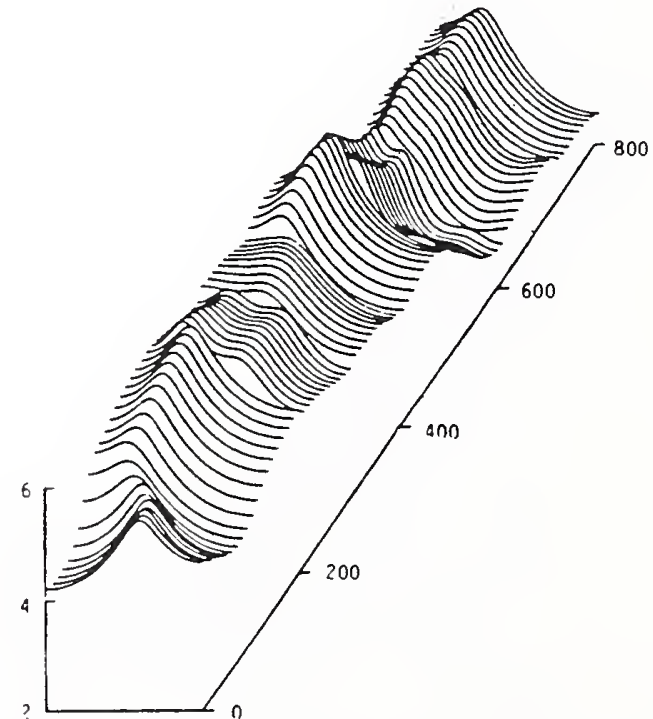

Fig. 3.11b Instantaneous spectrum for the simulated 1949 Olympia earthquake (from Kitagawa and Gersch, 1985). 


\subsubsection{Summary}

Several different methods have been presented to directly assess non-stationary data. These methods cover a wide range, from transforming the data into a stationary signal to full-fledged non-linear, non-stationary analysis. The method used will depend on the nature of the data available and the nature of the information needed to be gleaned. Transforming the data (Polhemus and Cakmak, 1981) is an acceptable method if the non-stationarity is mild and mostly in the amplitude. This method does not give much information as to the time-varying change in frequency and damping.

Segmentation of the data into stationary pieces gives very good results and is widely used. The approach by Gersch and Brotherton (1982) is the most rational. It makes the best use of the limited data available and allows the noise model to change through time. However, if the process of interest has time varying parameters, then a recursive technique, such as presented by Kitagawa (1983), must be used. In fact, the extended Kalman filter is often used in other fields to estimate smoothly varying non-linear parameters.

\subsection{Estimation of Soil Parameters From Earthquake Strong Motion Data \\ 3.4.1 Introduction}

Up to this point, the work presented has been of a theoretical bent, to show the methods available for estimating system parameters. Understanding these discussions will allow the reader to understand actual field applications. In this section, actual field experiments made using earthquake strong motion data are presented.

\subsubsection{Use of Shear Beam Theory}

An early success using measured response of an earth dam to earthquake excitation to estimate soil properties was made for the Santa Felicia dam in California (Abdel-Ghaffar and Scott, 1979). The input (base displacement) and output (crest motion) of the structure was recorded for the 1971 San Fernando earthquake, and another temblor in $1976\left(\mathrm{M}_{\mathrm{L}}=\right.$ 3.7). A shear-beam theory model was used to estimate shear moduli and damping ratio for the rolled-fill earth dam, constructed from gravelly sands.

The soil was modeled as a hysteretic SDOF system with a non-linear restoring force. An estimate of the hysteretic response can be made by plotting the crest displacement relative to the base, against absolute acceleration of the dam. Using the shear-beam model, the slope and area of the hysteresis loop can correspond to shear modulus and damping ratio (see Richart et al, 1970). Correlations can also be made to a finite element model using estimated parameters. The fundamental frequency behavior of the dam was isolated by narrow band-pass filtering the data. Without this filtering, the plotted hysteresis loops 
were erratic and useless for analysis. The precariousness of this modeling can be appreciated from the very small displacement of the top of the dam relative to the base $2.0 \mathrm{~mm}$.

The non-stationary response of the dam was analyzed by breaking the record into approximately one-second segments. Using both two-dimensional shear-beam theory (with constant shear modulus) and one-dimensional theory (shear modulus varying with depth), a relationship for dynamic stiffness, $G$, was derived. For the two-dimensional case the relationship for $\mathrm{G}$ is given by :

$$
G=\frac{\tau_{11}}{\gamma_{11}}=\rho\left(\frac{v_{11}}{\omega_{11}}\right)^{2} \cdot \frac{(\ddot{x}+\ddot{z})_{\max }}{x_{\max }}
$$

$$
\text { where } \begin{array}{ll}
\tau_{11} & =\text { fundamental mode shear stress } \\
\gamma_{11} & =\text { fundamental mode shear strain } \\
\rho & =\text { mass density of dam soil } \\
\mathrm{v}_{\mathrm{s}} & =\text { shear velocity of dam soil } \\
\omega_{11} & =\text { fundamental mode natural frequency } \\
\mathrm{X}_{\max } & =\text { maximum relative displacement of hysteresis loop. } \\
\left(\tilde{x}+\tilde{z}_{\max }\right. & =\text { maximum absolute acceleration of hysteresis loop }
\end{array}
$$

A similar relationship exists for the one-dimensional case. In addition, expressions for stress and strain at any time were derived.

With these equations the relationship between shear modulus and shear strain can be calculated. Additional information is given by the data from the second, smaller earthquake. An example of the results is shown in Fig. 3.12, where the stiffness reduction values calculated are well within the range of those expected. However, a similar analysis for the damping ratio yielded a curve of different shape than expected $-S$-shaped rather than hyperbolic, as shown in Fig. 3.13. The discrepancy might be due to the fact that the soils tested were predominantly gravels, and there have been virtually no laboratory tests run on gravel, and the results were accurate. However, it has been seen throughout Chapt. 2 and 3 that the estimations for damping are often inaccurate and inexact. 


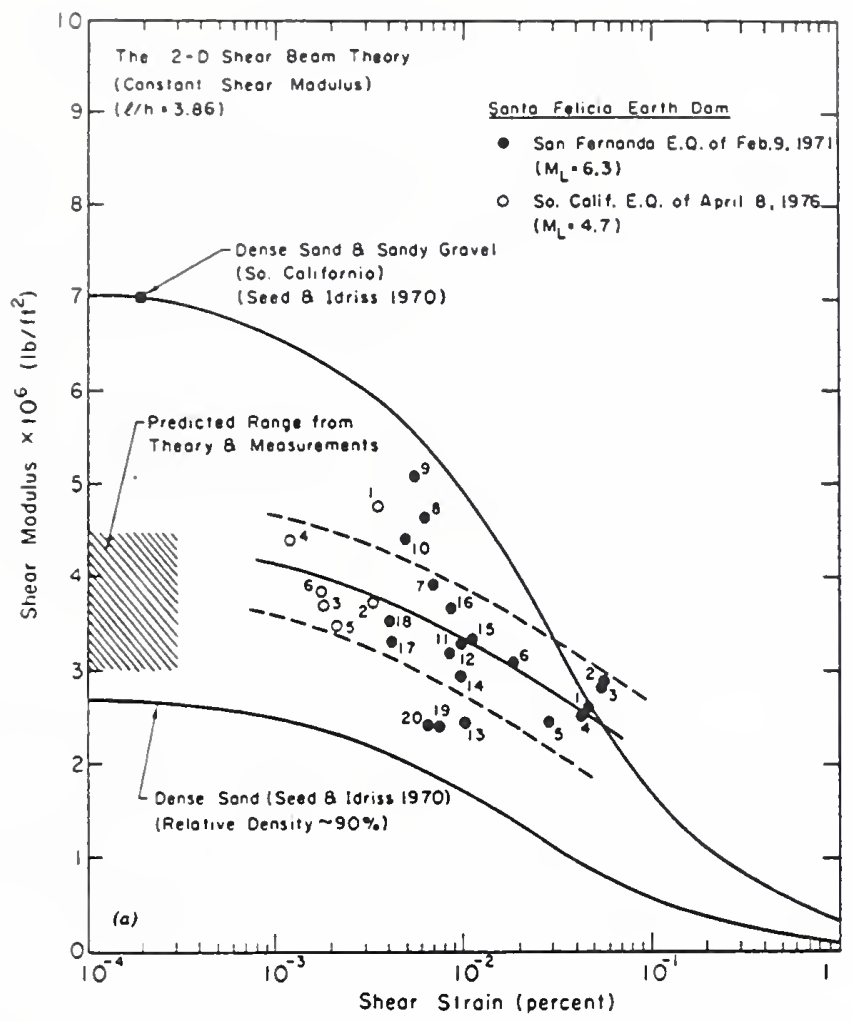

Fig. 3.12 Experimentally measured modulus degradation compared to previous values (from Abdel-Ghaffar and Scott, 1979).

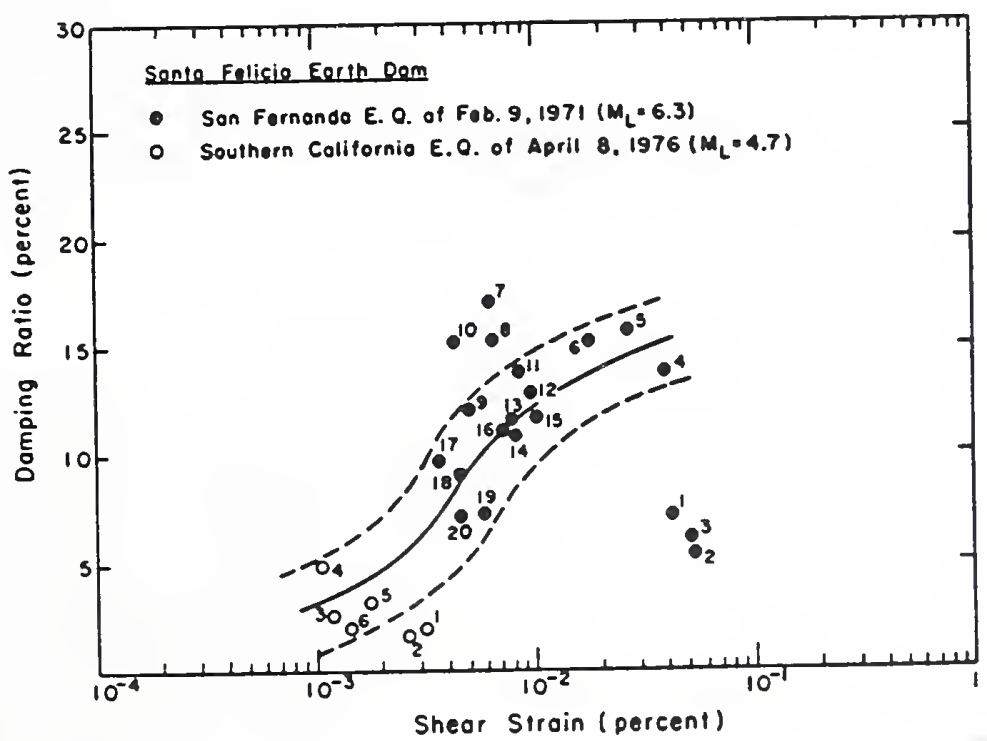

Fig. 3.13 Experimentally measured damping ratios (from Abdel-Ghaffar and Scott, 1979). 


\subsubsection{Fourier Analysis of Non-stationary Ground Motion}

A study has been made of data recorded at the Wildlife site in the Imperial Valley during the 1987 Superstition Hills earthquake (Vucetic and Zorapapel, 1990). For this event, three-dimensional accelerograms were recorded at a depth of 7.5 meters at the surface. In addition, pore water pressure was monitored throughout the shaking. The paper attempts to correlate a decrease in natural frequency of the soil layer, associated with degradation of the shear modulus, with the increase of pore water pressure as the condition of liquefaction is approached. However, it has been postulated that the piezometers were not functioning properly (Hushmand et al., 1992, 1991). The methods used in the analysis are instructive, in addition to giving a view of the state-of-the-practice in the use of system identification in the geotechnical field.

The system is well-defined for this problem, since both the input and output are known. Rather than use the more certain AR methods discussed in Chapt. 2, the authors choose to characterize the motions by simple Fourier spectra, with all the attendant uncertainty and problems discussed in the previous chapter. The system response function is not calculated directly through a least squares deconvolution or system identification procedure, rather is characterized by the spectral ratio. Because it is a simple ratio, a small error in one of the spectral estimates can have a very large effect on the calculated value.

The non-stationarity of the data is taken into account by segmenting the data, although the segmenting is not based on preserving sections with a common variance. Instead, the segmentation is based on physical concerns such as arrivals of various wave modes, or changes in pore water pressure. The analysis showed that the fundamental period of the soil layer lengthened as the pore pressure built up and the soil stiffness degraded. The entire process was also modeled using the DESRAMOD computer program (Vucetic, 1986) which calculates soil and pore water behavior based on a one-dimensional lumped parameter model. The resonant frequencies calculated by DESRAMOD and those from the Fourier analysis agreed well, especially for the vertical component.

\subsubsection{Parameter Estimation Using Impedance Functions}

Actual earthquake excitation has been used to measure experimental impedance functions of structures for comparison to theoretical functions, with acceptable results (Mau and Wang, 1990; Loh and Mau, 1989). Loh and Mau studied the modal behavior of the one-quarter scale model nuclear power plant containment vessel in Lotung, Taiwan. The rigid structure is very well instrumented so that the rigid body motion could be very well characterized with simple computation. The paper reports preliminary work, for which the free-field motion is not taken into account. The best fit between the theoretical impedance functions and the experimental impedance functions was for the assumed material values given in Table 3.2. 
Table 3.2 Estimated Material Parameters for Lotung Site (after Loh and Mau, 1989).

\begin{tabular}{c|c} 
Natural Frequency & $2.72 \mathrm{~Hz}$ \\
\hline Damping Ratio & $10.9 \%$ \\
\hline Shear-wave Velocity & $310 \mathrm{~m} / \mathrm{s}$
\end{tabular}

Mau and Wang (1990) continue the work on the Lotung containment structure with a more complete analysis where the form of the impedance function was not assumed. Again, only the rocking impedance was studied to simplify the embedded foundation problem. The coupling terms are ignored, allowing the system to be modeled as a singleinput-single-output system, with impedance function acting as the transfer function (filter). The kinematic input motions must then be calculated from the measured motions.

The dynamic impedance is calculated using the method of Bendat and Piersol (1986), discussed in Chapt. 2, Sect. 2.2.1. The transfer function is calculated from the ratio of the cross-spectrum to the autospectrum (Eq. 2.3). The coherence function was also calculated, and only relationships for frequencies with high coherence $(1.5-4.5 \mathrm{~Hz})$ were used to define the impedance function. The results were very poor, possibly because the assumptions made about lack of coupling were incorrect, or more than one mode must be included in the analysis to obtain useful results.

\subsubsection{Non-linear Ground Response Analyses}

Possibly the best set of data for earthquake excitation of soils exists for the test site operated by the Electric Power Research Institute (EPRI) and the Taiwan Power Co. at Lotung Taiwan (Tang, 1987). At this site, two locations are instrumented with threecomponent accelerometers at depths of $47,17,11,6$ meters, and at the surface. One array is in the free-field while the other is adjacent to the one-quarter scale nuclear containment vessel mentioned in Section 3.4.4. The site is also well instrumented with piezometers at various depths and locations. The simplified soil profile consists of $30-35 \mathrm{~m}$ of silty sand and sandy silt with some gravel, above clayey silt and silty clay. The water table is within half a meter of the ground surface. This area is seismically active, and many earthquakes of exhibiting a wide range of magnitudes have occurred since 1986.

A series of studies have been undertaken at Lotung using parameter identification to evaluate the non-linear response of soils due to strong motion (Chang et al., 1991, 1990, 1989). The authors note that outside of actual liquefaction sites, there has been no documented field evidence of the degradation of soil properties with increasing strain (Chang et al., 1989). One notable exception is the work done for the U.S. Nuclear Regulatory Commission (Shannon-Wilson and Agbabian, 1975). 
The first phase of the work concentrated on calculating a transfer function for the soil at several different points of the excitation history. The parameter of interest from the transfer function was resonant frequency. A simplified analysis for a two-layer system was used to calculate the effective S-wave velocity since the soil profile was known (Dobry at al., 1976). Shear moduli were calculated from the estimates of wave velocity. The strong motion record was divided in three sections: (1) initial motion before strain levels high enough to degrade the soil was reached, (2) peak motion, and (3) the coda or ring-down.

The transfer functions were estimated from the ratios of output Fourier spectra and input spectra from various depths. The Fourier spectra were smoothed by a triangular lag window. Unfortunately, the data lengths were very short since two of the time windows were only four seconds long. A Burg estimator would be much more appropriate for this application. In addition, use of an optimal segmentation scheme (Gersch and Brotherton, 1982) might be very helpful in identifying exactly when the parameters of the system actually changed.

The shear modulus showed a marked decrease from the initial low-level excitation to peak deformation. Depending on the depth used as input, the modulus reduction factor ranged from 0.60 to 0.14 . These values are for a magnitude 6.5 earthquake exhibiting a peak horizontal acceleration of $0.21 \mathrm{~g}$. As a check on reality, the S-wave amplitudes for the initial segment were in very good agreement with the actual measured values. A troublesome point is the large discrepancy in modulus reduction factor for the two horizontal components. The expectation would be that they would be virtually identical, since soil degradation is usually thought of as a scalar quantity.

This body of data is ideal for checking the results of ground response programs such as SHAKE (Schnabel et al, 1972) and DESRA-2 (Lee and Finn, 1985). SHAKE was tested in both the forward prediction mode and the inverse surface-to-depth mode, and DESRA-2 in the forward mode (Chang et al., 1990). The modulus degradation curve was calculated from actual field data in the manner discussed immediately above. The equivalent damping ratio was estimated from resonant column tests and the Seed-Idriss curve. For the SHAKE analyses, the additional input parameter was the field gathered S-wave velocity profile.

The results from SHAKE show that the calculated motions are higher than actual for frequencies greater than $0.6-1.5 \mathrm{~Hz}$, with the lower frequency associated with the analysis of a thicker layer of soil. There was no correlation for phase information. The backward analysis yielded better results than the forward propagation analyses. The disagreements might be due to the equivalent linear use of a single value of shear modulus per layer. The modulus used might only be valid for part of the load history, most specifically the peak strains for which the modulus is corrected for (Chang et al., 1990).

The results from the forward propagation non-linear DESRA-2 analysis show good agreement between actual and calculated displacement for frequencies up to about $6 \mathrm{~Hz}$. There was also good correlation for phase angle for frequencies up to about $3 \mathrm{~Hz}$. The 
shape of the equivalent damping ratio curve back-calculated from the non-linear analysis is different from that normally expected. Rather than the expected hyperbolic curve, the field curve is S-shaped. This uncommon S-shaped curve is the same shape as that calculated by Abdel-Ghaffar and Scott (1979) from actual field data (see Section 3.4.2).

Further work on the shear modulus reduction curve based on actual large strain measurement is reported by Chang et al. (1991). This study utilized seven different earthquakes with magnitudes ranging from 4.5 to 7.0 , and peak horizontal accelerations ranging from $0.03 \mathrm{~g}$ to $0.21 \mathrm{~g}$. As for the earlier report discussed above (Chang et al., 1989), shear modulus is estimated from S-wave velocities derived from resonant frequencies and soil layer thickness. Shearing strains are calculated from wave propagation theory using SHAKE.

In this study, the non-stationarity of the accelerograms are not taken into account, with the transfer functions being estimated for the entire time history at once. This means that the resultant resonant frequency estimated is an average value for the entire strain history of the earthquake. The earlier work showed that there can be a very substantial change in soil stiffness through time, so this approach seems incomplete. Of course, for small events with little or no degradation, the seismic velocities and the back calculated values match very well.

The equivalent stiffnesses or S-wave velocities calculated from the transfer functions, are input into the computer program SHAKE, along with damping curves from laboratory tests. Shearing strains are calculated when recorded surface motions are run through SHAKE. The calculated strains are open to doubt since the program makes its estimation from the peak stress value, and calculated strain and stress at different points in the soil layer. All the problems with the equivalent-linear approach brought up by Chang et al. (1990) are appropriate here too.

If the described method is accepted as valid, the resulting modulus reduction curves are as shown in Fig. 3.14, along with a comparison to laboratory test results. The results show that for small strains (surface acceleration $<0.03 \mathrm{~g}$ ), the results from geophysical methods, resonant column, and system identification are in good agreement. For intermediate strains $\left(5 \times 10^{-3}\right.$ to $\left.2 \times 10^{-2}\right)$ the back calculated values for modulus reduction are up to twenty percent lower than the resonant column measurements. For higher strains of $3 \times 10^{-2}$ to $2 \times 10^{-1}$, the results from cyclic triaxial tests are in fair agreement with the field values for shear modulus, with moderated scatter for the laboratory data.

This field test is the most complete to date. However, some compromising assumptions should be mentioned. The values of S-wave velocity and strain are average values for the entire event, from low stress to peak and back, and not actual values for any particular part of the excitation history. The method of calculating the transfer function is can amplify inaccuracies, since the high variance in Fourier estimates can cause even larger errors when the ratio is taken. The 


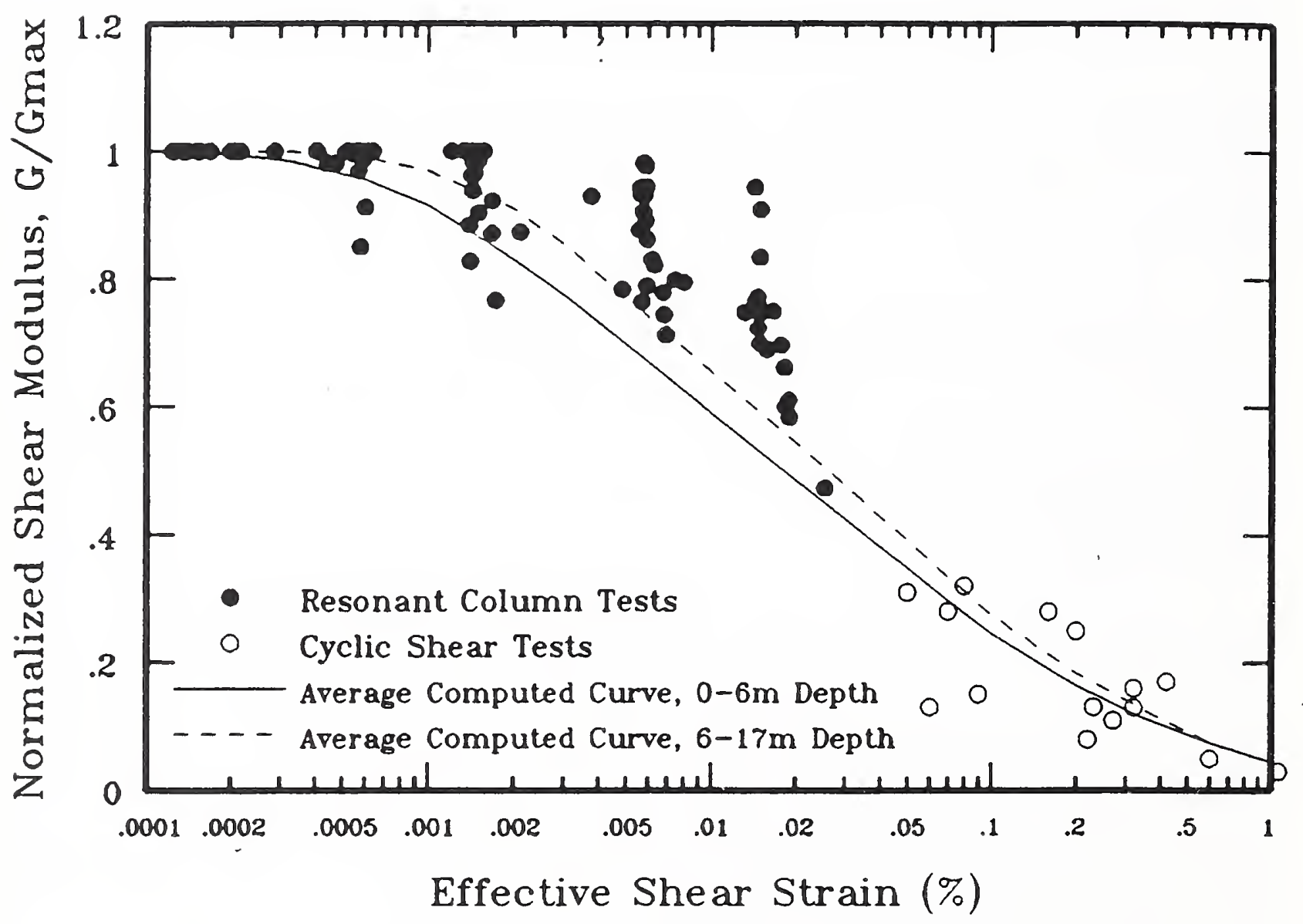

Fig. 3.14 Back-calculated normalized shear modulus compared with laboratory test data (from Chang et al., 1991). 
use of SHAKE to calculate strain raises questions, especially given the problems discussed by the authors one year earlier (Chang et al., 1990). It would be very informative to use this fine set of data with a more thorough and challenging set of analyses.

Due to the general lack of data sets for which the ground motion input into a soil profile is known from downhole instruments, an attempt has been made to simplify the inverse problem so that only the output (surface) strong motion needs to be known (Tokimatsu et al., 1989). As in the attempt by Nakamura (1989), an assumption has to be made as to the character of the input motion, since the system can not be solved for with only the output known.

Tokimatsu assumes one effective soil layer over bedrock. Working from the basic equation linking S-wave velocity and fundamental period (Dobry et al., 1976),

$$
V_{s}=\frac{4 H}{T} .
$$

An expression for the shear modulus is derived as

$$
G=16 \rho\left(\frac{H}{T}\right)^{2}
$$

where $\mathrm{H} \quad=$ thickness of the soil layer

$\rho \quad=$ mass density of the soil layer

$\mathrm{T} \quad=$ fundamental period of the soil layer

The assumption is now made that any increase in the fundamental period is directly related to a decrease in soil layer stiffness:

$$
\frac{G}{G_{\max }} \propto\left(\frac{T_{\max }}{T}\right)^{2}
$$

where $\mathrm{G} / \mathrm{G}_{\max } \quad=$ modulus reduction factor

$\mathrm{T}_{\max }=$ fundamental period of the soil layer at $10^{-6}$ shear strain.

Assuming linear behavior of the soil mass, a relation for the shear displacement at any depth $z$ from peak particle velocity and S-wave velocity is given. This derivation assumes that the shear strain at any depth is just a time-offset displacement of the surface strain. Although Tokimatsu claims now to be able to accurately calculate shear strain at any point in the soil mass at any time, he defines an effective shear strain to be 85 percent of the maximum shear strain. This value is picked since for the first ten seconds of the excitation, the effective strain is considered to be 85 percent of the maximum shear strain.

For the estimation of damping ratio, Tokimatsu assumes that the input motions from the bedrock layer are white noise, a poor assumption based on what has been presented in this report. This assumption, however allows the authors to say that the Fourier spectrum of the surface motions has much the same shape as the spectral ratio (i.e. amplification spectrum). 
The authors claim good correspondence between laboratory results and their technique. However, until a more rational and complete derivation is given, this approach is not considered useful. There are too many theoretical problems and shaky assumptions involve to accept it as presented.

A good example of the application of time-adaptive modeling of soil parameters from earthquake strong motion data is a paper by Safak (1989). While the paper is difficult to understand, the application of advanced system identification techniques is well illustrated. In this paper the adaptive scheme uses an exponentially decaying "forgetting-factor" to take the non-stationarity into account, rather than a full Kalman filter that actually models the changing parameters directly.

The process is modeled as a single input-single output system where the input and output are known, but the auxiliary noise input is not known but assumed to be white. The general model is autoregressive-moving average with a moving average auxiliary input (ARMAX). The ARMA parameters are estimated by a recursive least squares scheme known as the Recursive Prediction Error Method. Safak shows that for earthquake ground motions, a subset of the ARMAX, the ARX (autoregressive with noise) is most appropriate. If the prediction error series is a stationary Gaussian variable with zero mean, this method is identical to the Maximum Likelihood estimation.

Proper application of a parametric model to a process requires determining the proper model order. The simplest criterion is to use the order that minimizes the prediction error

$$
\epsilon(t)=y(t)-\hat{y}(t)
$$

where $\epsilon(\mathrm{t}) \quad=$ prediction error at time $\mathrm{t}$ (innovation)

$y(t) \quad=$ actual output at time $t$

$\hat{y}(t)=$ prediction of output at time $t$ made at time $t-1$.

As discussed in Chapter 2, this estimate can be checked by observing whether the prediction error time series is white. It was shown that if all available information is retrieved from the data, the residuals will become a white noise series. However, it is possible for the system to be correctly modeled while the noise is not. This case still will give the correct transfer function but not have a white residuals series. The validity of the model can still be checked by observing the cross-correlation of the input and residuals series. For a valid model, there will be no correlation between the two since the model "pulls" all the information out, leaving only the noise. The final choice is to use the AIC, which maximizes the entropy between the model and actual process.

Safak gives an example of the ARX approach to spectral estimation by analyzing the surface strong motion from the 1971 San Fernando earthquake. If the input white noise signal is combined with the auxiliary white noise signal, the ARX model becomes the more familiar ARMA model. This method is identical to the Burg estimation if the MA 
parameters (which model sharp dips) are not included. As was shown in Chapter 2, this method avoids making the assumption that the signal is stationary and the noise process is discrete from the process of interest. Figure 3.15 shows the resulting instantaneous spectral estimates for one, five, and eighteen seconds into the earthquake (note the log-log scale). It is seen that the fundamental frequency becomes smaller though the shaking.

The ARMAX model was also applied to estimating the parameters of a soil column (Safak, 1989). In this case nearby bedrock motion and the surface motion were both known for a site subjected to the 1985 Chilean earthquake. The estimated parameters are associated with system fundamental frequency and damping ratio. In the frequency domain the spectrum shows frequency-dependant amplification. The method used allows the process parameters to be estimated throughout time so that the change in the system can be monitored. In this paper only the results for eighteen seconds into the temblor, the coda, is reported. The results for the first mode are a natural frequency of $1.35 \mathrm{~Hz}$ and a damping ratio of 11.9 percent.

The results in this paper are arrived at through a deliberate, rational approach rather than trial-and-error, ad hoc methods. Obvious areas of improvement would be to incorporate some of the techniques developed by Gersch and his cohorts. The author himself points out that the ARMAX model did not model the noise very well, and suggests the use of the Box-Jenkins model where the noise is modeled with it's own ARMA polynomial. Safak also points out that the application of a Kalman filter would take the non-stationarity more rationally into account. 


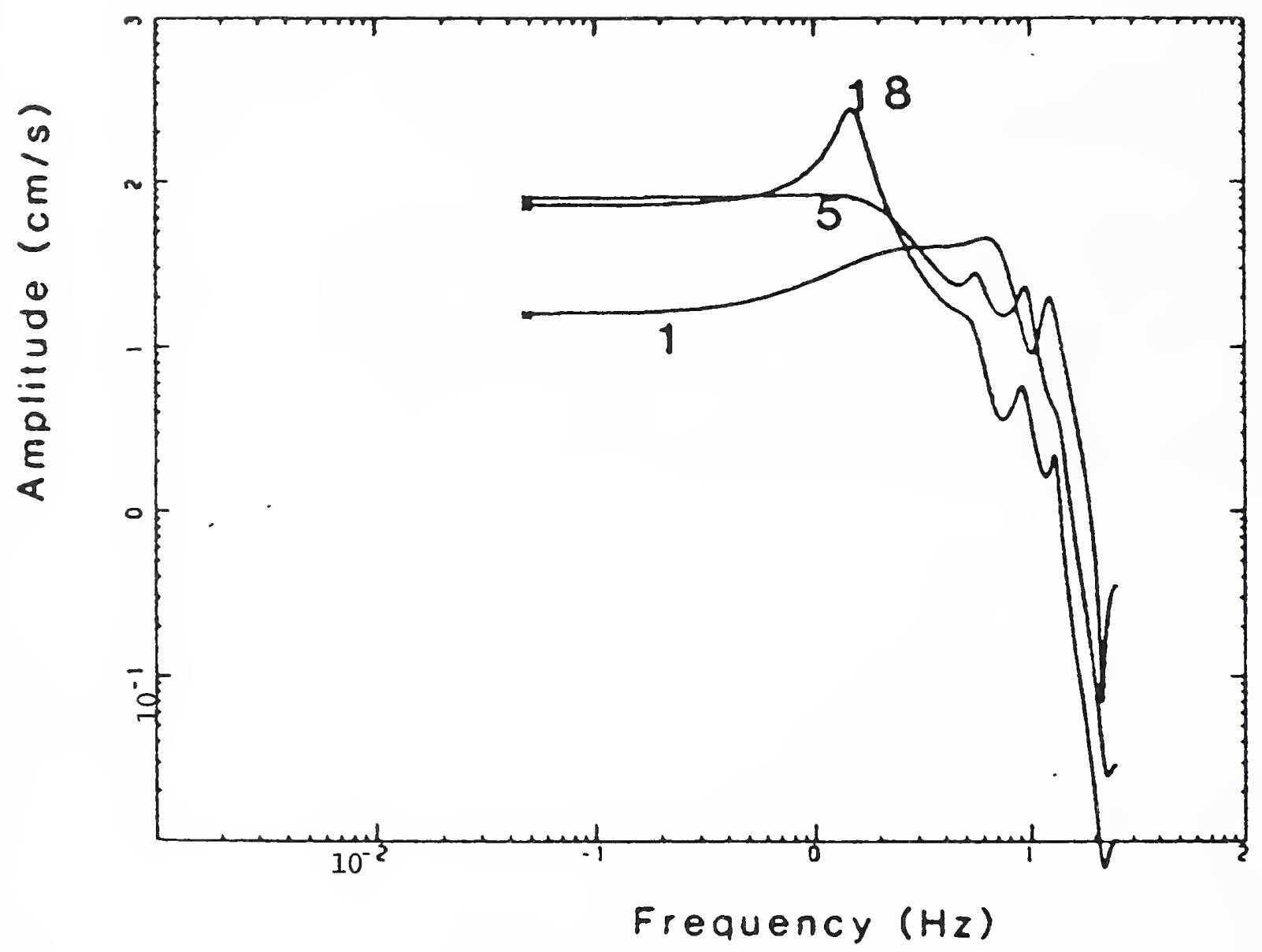

Fig. 3.15 Amplitude of the transfer functions of the 1971 San Fernando temblor modeled as an ARMA $(8,7)$ process at times $t=1,5$, and 18 seconds (from Safak, 1989). Note log-log scale. 


\section{CHAPTER 4 CONCLUSIONS AND SUMMARY}

\subsection{A Framework of Knowledge \\ 4.1.1 Estimation Techniques}

For the system identification problems of interest to this report, the parameters to be found are system damping and resonant frequency. The resonant frequency is associated with the peak of the amplitude spectrum. It has been seen that a single peak is relatively easy to identify, especially if the proper model has been used for the process. For the very large resonance peak of the principle harmonic, virtually any method will give a close result, even for a relatively non-stationary case. However, every method distorts the shape of the peaks, the usual source of the damping estimate. It is therefore to be expected that system identification will give good estimates of modal frequencies, but poor estimates of damping.

A study was done by Gersch (1974) in order to determine the greatest degree of accuracy with which a proper order ARMA model can estimate the damping ratio and natural frequency of a structure, using the Maximum Likelihood method. He notes that as the number of data points $(\mathbf{N})$ becomes large, the estimate approaches the actual model and approaches the Cramer-Rao lower bound of variance. The accuracy is given as the coefficient of variation - standard deviation divided by the mean. For both parameters, the coefficient of variation is inversely proportional to $\sqrt{\mathrm{N}}$ and length of the period sampled, and relatively insensitive to noise. For one thousand data points, the coefficient of variation was less than 0.01 for natural frequency, but greater than 0.2 for damping ratio; this is an order of magnitude difference.

As a note of caution, while the more complicated models encompass more of a process in a rational manner, the values needed as input must often be assumed, making the results less meaningful. The results of the more complicated models should be compared to those of simpler, more intuitive calculations that allow the engineer to maintain a "feel" for the validity of the results throughout the evaluation process. In the words of G. E. P. Box, "All models are incorrect, but some are more useful than others."

\subsubsection{Conclusions and Recommendations}

Theoretical and practical considerations have shown that transforming a data series into the frequency domain is not a straight-forward process. The results of a simple DFT has variance equal to the mean, and is very severely corrupted by leakage. This has resulted in the tremendous amount of ad hoc attempts to improve on the bias and uncertainty problems, lumped in this paper as the "classical approach". This presentation has not gone into detail about these methods, except to become aware of the inherent problems. A thorough summary and comparison of almost all these non-parametric methods, and parametric methods, can be found in Kay and Marple (1981). Included Kay and Marple's 
paper is a shocking comparison of the results of all the methods, each giving results unrelated to the others, and all different from the theoretical frequency spectrum.

These problems were directly addressed by Thomson (1982) in deriving the multitaper method. It is believed that this approach is the "best" non-parametric spectral approach if proper pre-treatment of the signal is done. These same considerations are addressed from a philosophical point of view by many papers of John Tukey (e.g. Tukey, 1984; Brillinger and Tukey, 1984). Blindly applying the FFT to one's data is a very dangerous thing to do, and the cautions and insights of Tukey should be taken to heart before starting.

If the process being studied can be modeled as an AR or ARMA process, then the parametric approach is the best method to characterize the system. The Burg method is ideal for short, relatively stationary data. The growing family of adaptive and Kalman filters are proving themselves with non-stationary processes. The AR model was shown to be initially derived for a SDOF oscillator, and the ARMA model can be derived directly from the differential equation of motion for an $\mathrm{N}$-degree-of-freedom system, with the damping ratio and resonant frequency the model parameters (e.g. Gersch and Luo, 1970). A 2n-2n ARMA model is therefore a valid model for a layered soil system, or soil-structure interaction problem.

The examination of the various attempts to characterize soil properties through analysis of the response to earthquake excitation has illustrated some of the main pitfalls and advantages of using the system identification approach. One important problem in evaluating the various methods is that there is no "correct" value against which to compare the results. The mechanical engineer has the advantage of being able to construct a system similar to that being tested, with known parameters against which to test the method. The geotechnical engineer never has this luxury. This limitation highlights one of the strengths of the SI approach, since there is no other way to actually measure the in situ fundamental frequency and damping ratio for strains even remotely approaching those encountered during earthquake loading.

The geotechnical community has not utilized the methods, approaches, or warnings discussed by the system identification community and presented in Chapter 2. The methods used to date make no acknowledgement to the non-stationarity of the signals, or that the Fourier spectral estimate may have any limitations or uncertainties. The exceptions to this are the researchers using forced vibration and fitting impedance functions.

The forced vibration-impedance function method is very attractive since the investigators have control over the input signal. Besides simplifying the calculations, this avoids having to wait many years for an earthquake to occur at a given site. The approach is also straight-forward in concept. The major problem seems to be an inability of generating enough energy to involve a deep column of soil. The strains induced in the soil will be in the low range for the same reason. 
It is recommended that the complete input-output data sets from the Wildlife site and Lotung be analyzed using the tools provided by the researchers in control. The improved segmentation scheme of Gersch and Brotherton (1982) is very appealing if the process parameters are not believed to change during excitation. For situations where the soil is believed to have undergone degradation, methods taken after Kitagawa and Gersch (1985) seems to offer the most promise. In any event, application of AR and ARMA spectral estimators will yield spectral estimates with higher certainty than Fourier analysis. 


\section{CHAPTER 5 BIBLIOGRAPHY}

Abdel-Ghaffar, A. M., \& Scott, R. F. (1979). Shear moduli and damping factors of earth dam. Journal of the geotechnical engineering division, ASCE, 105(GT12), pp. 1405-1426.

Akaike, H. (1981). Modern development of statistical methods. Trends and progress in system identification (ed. Eykhoff, P.) Elmsford, NY: Pergamon Press.

Akaike, H. (1974). A new look at the statistical model identification. IEEE Transactions on

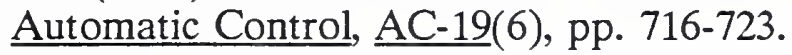

Akaike, H. (1970). Statistical predictor identification. Annals of the Institute of Statistical Mathamatics, 22, pp. 203-217.

Aki, K., \& Richards, P. G. (1980). Quantitative seismology. 1 (p. 137). San Francisco: W. H. Freeman.

Astrom, K. J. (1980). Maximum likelihood and prediction error methods. Automatica, 16 , pp. 551-574.

Astrom, K. J., \& Eykhoff. (1971). System identification - a survey. Automatica, 7(2), pp. 123-162.

Bendat, J. S. (1990). Nonlinear system analysis and identification of random data. New York: Wiley.

Bendat, J. S., \& Piersol, A. G. (1986). Random data. (2nd.) New York: Wiley Interscience.

Bloomfield, P. (1976). Fourier analysis of time series: an introduction. (pp. 118-150). New York: Wiley.

Bohlin, T. (1987). Model validation. Encyclopedia of systems and control (Singh, M.) Oxford: Pergamon Press.

Box, G. E. P., \& Jenkins, G. M. (1976). Time series analysis: forcasting and control. San Francisco: Holden-Day.

Bracewell, R. N. (1978). The fourier transform and its applications. New York: McGraw-Hill.

Brigham, E. O. (1974). The fast Fourier transform. Englewood Cliffs, NJ: Prentice-Hall. 
Brillinger, D. R. (1988). Some statistical methods for random process data from seismology and neurophysiology. The annals of statistics, 16(1), pp. 1-54.

Brillinger, D. R., \& Tukey, J. W. (1984). Spectrum analysis in the presence of noise: some issues and examples. in D. R. Brillinger (ed.), The Collected Works of John W. Tukey. II (pp. 1001-1141). Belmont, CA: Wadsworth.

Burg, J. P. (1975). Maximum entropy spectral analysis. Doctoral dissertation, Stanford University, Palo Alto, CA.

Cadzow, J. A. (1982). Spectral estimation : an overdetermined rational model equation approach. Proceedings of the IEEE, 70(9), pp. 907-939.

Cakmak, A. S., \& Sherif, R. I. (1984). Parametric time series models for earthquake strong motions and their relationship to site parameters. Proceedings, eighth world conference on earthquake engineering. II San Francisco, CA. (pp. 581-588). Englewood Cliffs: Prentice-Hall.

Chang, C.-Y., Mok, C. M., Power, M. S., Tang, Y. K., Tang, H. T., \& Stepp, J. C. (1991). Development of shear modulus reduction curves based onLotung down-hole ground motion data. S. Prakash, Second international conference on recent advances in geotechnical earthquake engineering and soil dynamics. I St. Louis, MO. (pp. 111-118). Rolla, MO: University of Missouri-Rolla.

Chang, C.-Y., Mok, C. M., Power, M. S., Tang, Y. K., Tang, H. T., \& Stepp, J. C. (1990). Equivalent linear versus nonlinear ground response analyses at Lotung seismic experiment site. Proceedings of the fourth U.S. national conference on earthquake engineering. 3 Palm Springs, CA. (pp. 327-336). Oakland, CA: EERI.

Chang, C.-Y., Power, M. S., Tang, Y. K., \& Mok, C. M. (1989). Evidence of nonlinear soil response during a moderate earthquake. Proceedings of the twelfth international conference on soil mechanics and foundation engineering. 3 Rio De Janeiro. (pp. 1927-1930). Rotterdam: Balkema.

Chang, M. K., Kwiatkowski, J. W., Nau, R. F., Oliver, R. M., \& Pister, K. S. (1982). ARMA models for earthquake ground motion. Earthquake engineering and structural dynamics, 10, pp. 651-662.

Crede, C. E. (1957). Vibration and shock isolation. (pp. 176-186). New York: Wiley.

Crouse, C. B., \& Hushmand, B. (1987). Estimation of bridge foundation stiffness from forced vibration data. in A. S. Cakmak, Soil dynamics and liquefaction. (Developments in geotechnical engineering 42). (pp. 123-136). Amsterdam: Elsevier. 
Crouse, C. B., Hushmand, B., Luco, J. E., \& Wong, H. L. (1990). Foundation impedance functions: theory vs. experiment. Journal of the geotechnical engineering division, ASCE, $116(3)$, pp. 432-449.

Dargahi-Noubary, G. R., Laycock, P. J., \& Rao T. Subba. (1978). Non-linear stochastic models for seismic events with applications in event identification. Geophysics journal of the royal astronomical society, $\underline{55}$, pp.655-668.

Dobry, R., Oweis, I., \& Urzua, A. (1976). Simplified procedures for estimating the fundamental period of a soil profile. Bulletin of the seismological society of america, 66(4), pp. 1293-1321.

Falconer, D. D., \& Ljung, L. (1978). Application of fast-Kalman estimation to adaptive equalization. IEEE transactions on communications, COM-26, pp. 1439-1446.

FEMA. (1991). EQE Inc, ACT-25, Seismic vulnerability and impact of disruption of lifelines in the coterminous United States. (FEMA 224). Washington, D.C.: Federal Emergency Management Agency.

Finn, W. D. Liam. (1991). Geotechnical Engineering Aspects of Microzonation. in Proceedings, fourth international conference on seismic zonation. 1 Stanford, CA. (Earthquake Engineering Research Institute) (pp. p.199-259). Oakland, CA: EERI.

Florin, V. A., \& Ivanov, P. L. (1961). Liquefaction of saturated sandy soils. Proceedings of the fifth international conference on soil mechanics and foundation engineering. I Paris. (pp. 107-111). Paris: Dunod.

Gazetas, G. (1983). Analysis of machine foundation vibrations: state of the art. Soil dynamics and earthquake engineering, 2(1), pp. 2-42.

Geckinli, N., \& Yavuz, D. (1978). Some novel windows and a concise tutorial comparison of window families. IEEE transactions on acoustics, speech, and signal processing, ASSP-26(6), pp. 501-507.

Gersch, W. (1974). On the achievable accuracy of structural system parameter estimates. Journal of sound and vibration, 34(1), pp. 63-79.

Gersch, W., \& Brotherton, T. (1982). Estimation of stationary structural system parameters from nonstationary random vibration data: a locally stationary model method. Journal of sound and vibration, 81(2), pp. 215-227.

Gersch, W., \& Kitagawa, G. (1985). A time varying AR coefficient model for modelling and simulating earthquake ground motion. Earthquake engineering and structural dynamics, 13 , pp. 243-254. 
Gersch, W., \& Luo, S. (1972). Discrete time series synthesis of randomly excited structural system response. Journal of the acoustic society of america, 51(1), pp. 402-408.

Gersch, W., \& Martinelli, F. (1979). Estimation of structural system parameters from stationary and non-stationary ambient vibrations: an exploratory-confirmatory analysis. Journal of sound and vibration, 65 , pp. 303-318.

Gersch, W., Nielson, N. N., \& Akaike, H. (1973). Maximum likelihood setimation of structural parameters from random vibration data. Journal of sound and vibration, 31(3), pp. 295-308.

Graupe, D. (1989). Time series analysis, identification and adaptive filtering. (2nd. ed.) Malabar, FL: Robert E. Krieger.

Gutowski, P. R., Robinson, E. A., \& Treitel, S. (1978). Spectral estimation: fact or fiction. IEEE Transactions on Geoscience Electronics, GE-16(2), pp. 80-84.

Hannan, E. J. (1976). The identification and parameterization of ARMAX and state space forms. Econometrica, 44(4), pp. 713-723.

Hardin, J. C. (1986). Introduction to time series analysis. (NASA RP-1145). Washington, D.C.: National Aeronautics and Space Administration.

Holzer, T. L., Youd, T. L., \& Hanks, T. C. (1989a). Dynamics of liquifaction during the 1987 Superstition Hills, California, earthquake. Science, 244, pp. 56-59.

Hushmand, B., Scott, R. F., \& Crouse, C. B. (1992). In-place calibration of USGS pore pressure transducers at Wildlife Liquefaction Site, California, USA. Proceedings of the Tenth World Conference on Earthquake Engineering. 3 Madrid. pp. 1263-1268. Rotterdam: Balkema.

Hushmand, B., Scott, R. F., \& Crouse, C. B. (1991). In-place calibration of USGS pore pressure transducers at Wildlife Liquefaction Site, California, USA. Recent advances in instrumentation, data aquisition and testing in soil dynamics. G.S.P. 29, Orlando, FL. pp. 49-69. ASCE: New York.

Jurkevics, A., \& Ulrych, T. J. (1978). Representing and simulating strong ground motion. Bulletin of the seismological society of America, 68(3), pp. 781-801.

Kalman, R. E. (1960). A new approach to linear filtering and prediction problems. Transactions of the ASME, journal of basic engineering, (3), pp. 35-45.

Kalman, R. E., \& Bucy, R. (1961). New results in linear filtering and prediction theory. Transactions of the ASME, journal of basic engineering, (3), pp. 83-95. 
Kanasewich, E. R. (1981). Time sequence analysis in geophysics. Edmonton, Alberta: University of Alberta Press.

Kay, S. M., \& Marple Jr., S. L. (1981). Spectrum analysis - a modern perspective. Proceedings of the IEEE, 69(11), pp. 1380-1419.

Kitagawa, G. (1983). Changing spectrum estimation. Journal of sound and vibration, $\underline{89}(3)$, pp. 433-445.

Kitagawa, G., \& Gersch, W. (1985). A smoothness priors time-varying AR coefficient modeling of nonstationary covariance time series. IEEE transactions on automatic control, $\underline{\mathrm{AC}-30}$ (1), pp. 48-56.

Kozin, F. (1977). Estimation and modelling of non-stationary time series. in L. C. Wellford $\mathrm{Jr}$, Proceedings of the symposium on applications of computer methods in engineering. 1 Los Angeles, CA. (pp. 603-612). Los Angeles, CA: School of Engineering, University of Southern California.

Lee, M. K. W.; Finn, W. D. Liam. (1978). DESRA-2. Dynamic effective stress response analysis of soil deposits with energy transmitting boundary including assessment of liquefaction. Vancouver, B.C.: Dept. Civil Engineering, University of British Columbia. (Soil Mechanics Series No. 38)

Levinson, N. (1947). The Wiener RMS (root mean square) error criteria in filter design and prediction. Journal of mathematics and physics, 25 , pp. 261-278.

Lin, J.-S. (1990). Nonlinear system identification of soils using extended Kalman filters. Proceedings of the fourth U.S. national conference on earthquake engineering. $\underline{3}$ Palm Springs, CA. (pp. 865-874). Oakland, CA: EERI.

Ljung, L. (1979). Asymptotic behavior of the extended Kalman filter as a parameter estimator for linear systems. IEEE transactions on automatic control, AC-24(1), pp. $36-50$.

Ljung, L. (1987). System identification: theory for the user. Englewood Cliffs, NJ: Prentice-Hall.

Ljung, L., Morf, M., \& Falconer, D. (1978). Fast calculation of gain matrices for recursive estimation schemes. International journal of control, 27(1), pp. 1-19.

Ljung, L., \& Soderstrom, T. (1983). Theory and practice of recursive identification. Cambridge, MA: M.I.T. press. 
Loh, C. H., \& Mau, S. T. (1989). Characteristics of a structure-foundation system. Proceedings of the sessions related to seismic engineering, at Structures congress '89. San Francisco. (pp. 209-216). New York: The Society.

Luco, J. E., \& Wong, H. L. (1992). Identification of soil properties from foundation impedance functions. Journal of the geotechnical engineering division, ASCE, 118(5), pp. 780-795.

Luco, J. E., \& Wong, H. L. (1990). Identification of soil properties from vibration tests of small footings. Proceedings of the fourth U.S. national conference on earthquake engineering. $\underline{3}$ Palm Springs, CA. (pp. 875-884). Oakland, CA: EERI.

Marple Jr., \& S. Lawrence. (1987). Digital spectral analysis with applications. Englewood Cliffs: Prentice-Hall.

Mau, S. T., \& Wang, S. (1990). Identification of foundation impedance from earthquake records. Proceedings of the fourth U.S. national conference on earthquake engineering. $\underline{3}$ Palm Springs, CA. (pp. 885-892). Oakland, CA: EERI.

McClellan, J. H. (1982). Multidimensional spectral estimation. Proceedings of the IEEE, 70(9), pp. 1029-1036.

Munk, W. H., \& Snodgrass, F. (1957). Measurements of southern swell at Guadaloupe Island. Deep-sea research, 4 , pp. 272-286.

Murphy, J. R., Davis, A. H., \& Weaver, N. L. (1971). Amplification of seismic body waves by low-velocity surface layers. Bulletin of the seismological society of america, $61(1)$, pp. 109-145.

Nakamura, Y. (1989). A method for dynamic characteristics estimation of subsurface using microtremor on the ground surface. Quarterly report of railway technical research institute, $\underline{30}(1), \mathrm{pp} .25-33$.

Nau, R. F., \& Oliver, R. M. (1979). Adaptive filtering revisited. Journal of the operational research society, $30(9)$, pp. 825-831.

Newland, D. E. (1984). An introduction to random vibrations and spectral analysis. (2nd). New York: Longman.

Ohmachi, T., Nakamura, Y., \& Toshinawa, T. (1991). Ground motion characteristics of the San Francisco Bay area detected by microtremor measurements. S. Prakash, Second international conference on recent advances in geotechnical earthquake engineering and soil dynamics. II St. Louis, MO. (pp. 1643-1648). Rolla, MO: University of Missouri-Rolla. 
Otnes, R. K., \& Enochson, L. (1972). Digital Time Series Analysis. New York: Wiley.

Page, C. H. (1952). Instantaneous power spectra. Journal of applied physics, 23, pp. 103-106.

Polhemus, N. W., \& Cakmak, A. S. (1981). Simulation of earthquake ground motions using autoregressive moving average (ARMA) models. Earthquake engineering and structural dynamics, $\underline{9}$, pp. 343-354.

Popescu, T. D., \& Demetriu, S. (1990). Analysis and simulation of strong earthquake ground motions using ARMA models. Automatica, 26(4), pp. 721-737.

Priestley, M. B. (1988). Non-linear and non-stationary time series analysis. (pp. 139-183). San Diego, CA: Academic Press.

Priestley, M. B. (1967). Power spectral analysis of non-stationary random processes. Journal of sound and vibration, $\underline{6}(1), \mathrm{pp} .86-97$.

Radoski, H. R., Fougere, P. F., \& Zawalick, E. J. (1975). A comparison of power spectral estimates and applications of the maximum entropy method. Journal of geophysical research, $\underline{80}(4)$, pp. 619-625.

Richart, F. E., Hall Jr., J. R., \& Woods, R. D. (1970). Vibrations of soils and foundations. Englewood Cliffs, NJ: Prentice-Hall.

Robinson, E. A. (1982). A historical perspective of spectrum estimation. Proceedings of the IEEE, 70(9), pp. 885-907.

Safak, E. (1988). Analysis of recordings in structural engineering : adaptive filtering, prediction, and control. (Open-File Report 88-647). Menlo Park, CA: U.S. Geological Survey.

Safak, E. (1989). Optimal-adaptive filters for modelling spectral shape, site amplification, and source scaling. Soil dynamics and earthquake engineering, $\underline{8}(2)$, pp. 75-95.

Schnabel, P. B.; Lysmer, J.; Seed, H. B. (1972). SHAKE [Computer Program]. A computer program for earthquake response analysis of horizontally layered sites. Berkeley, CA: Earthquake Engineering Research Center. (No. 72-12)

Seo, K., Samano, T., Yamanaka, H., Xiansheng, H., Takeuchi, M., \& Kishino, Y. (1989). A simple procedure for predicting seismic motions with microtremor measurement. Proceedings of the national symposium on effects of surface geology on seismic motion. Tokyo, Japan. (pp. 207-212). 
Shannon \& Wilson - Agbabian Associates. (1976). In situ impulse test - an experimental and analytical evaluation of data interpretation procedures. (NUREG-0028). Washington, D.C.: U.S. Nuclear Regulatory Agency.

Shannon, C. E. (1948). A mathematical theory of communiation. Bell system technical journal, 27, pp. 379-423.

Silvia, T. M. \& Robinson, E. A. (1979). Deconvolution of geophysical time series in the exploration for oil and natural gas. (p. 251). Amsterdam: Elsevier.

Simmons, J. A. (1991). Deconvolution of acoustic emission and other causal time series. Journal of research of the national institute of standards and technology, 96(3), pp. 345-369.

Soderstrom, T., \& Stoica, P. (1989). System identification. New York: Prentice Hall.

Sorenson, H. W. (1970). Least-squares estimation: from Gauss to Kalman. IEEE spectrum, Z(7), pp. 63-68.

Swingler, D. N. (1979). A comparison between Burg's maximum entropy method and a nonrecursive technique for the spectral analysis of deterministic signals. Journal of

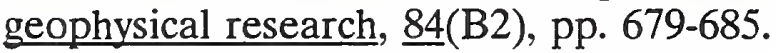

Thomson, D. J. (1982). Spectral estimation and harmonic analysis. Proceedings of the IEEE, 70(9), pp. 1055-1096.

Tang, H. T. (1987). Large-scale soil-structure interaction. (NP-5513-SR). Palo Alto, CA: EPRI.

Tokimatsu, K., Midorikawa, S., \& Yoshimi, Y. (1989). Dynamic soil properties obtained from strong motion records. Proceedings of the twelfth international conference on soil mechanics and foundation engineering. 3 Rio De Janeiro. (pp. 2015-2018). Rotterdam: Balkema.

Treitel, S., Gutowski, P. R., \& Robinson, E. A. (1977). Emperical spectral analysis revisited. in Topics in numerical analysis 3. (pp. 429-446). New York: Academic Press.

Tukey, J. W. (1984). When should which spectrum approach be used? in D. R. Brillinger (ed.), The Collected Works of John W, Tukey. II (pp. 981-1000). Belmont, CA: Wadsworth.

Udwadia, F. E. (1985). Some uniqueness results related to soil and building structural identification. SIAM journal of applied mathematics, 45(4), pp. 674-685. 
Udwadia, F. E., Sharma, D. K., \& Shah, P. C. (1978). Uniqueness of damping ans stiffness distributions in the identification of soil and structural systems. Journal of applied mechanics, 45 , pp. 181-187.

Ulrych, T. J., \& Clayton, R. W. (1976). Time series modelling and maximum entropy. Physics of the Earth and Planetary Interiors, 12, pp. 188-200.

Van Den Boom, A. J. W., \& Van Den Enden, A. W. M. (1974). The determination of the orders of process-and noise dynamics. Automatica, 10, pp. 245-256.

Vucetic, M. (1986). Pore pressure buildup and liquefaction at level sandy sites during earthquakes. Doctoral dissertation. Rensselaer Polytechnical Institute, Troy, NY. p. 611.

Vucetic, M., \& Zorapapel, G. T. (1991). The effects of liquefaction process on the spectral content of strong ground motion. T. D. O'Rourke, \& M. Hamada, Proceedings from the third Japan-U.S. workshop on earthquake resistant design of lifeline facilities and countermeasures for soil liquefaction. Technical Report NCEER-91-0001 San Francisco, CA. (pp. 403-418). Buffalo: National Center for Earthquake Engineering Research.

Welch, P. D. (1967). The use of fast Fourier transform for the estimation of power spectra: A method based on time averaging over short, modified periodograms. IEEE transactions, audio electro-acoustics, AU-15, pp. 70-73.

Wiener, N. (1964). Extrapolation, interpolation and smoothing of stationary time series. Cambridge, MA: M.I.T. press.

Yule, G. U. (1927). On a method of investigating periodicities in disturbed series, with special reference to Wolfer's sunspot numbers. Philosophical transactions of the royal society of London, Series A, 226, pp. 267-298.

Zhang, X., Ishibashi, I., \& Wang, L. R. L. (1991). Lateral stiffness and damping coefficient of soils for seismic analysis of buried pipelines. S. Prakash, Second international conference on recent advances in geotechnical earthquake engineering and soil dynamics. ISt. Louis, MO. (pp. 779-784). Rolla, MO: University of Missouri-Rolla. 


